\title{
Frozen percolation in two dimensions
}

\author{
Demeter Kiss
}

Received: 12 July 2013 / Revised: 10 November 2014 / Published online: 10 December 2014

(C) Springer-Verlag Berlin Heidelberg 2014

\begin{abstract}
Aldous (Math Proc Camb Philos Soc 128:465-477, 2000) introduced a modification of the bond percolation process on the binary tree where clusters stop growing (freeze) as soon as they become infinite. We investigate the site version of this process on the triangular lattice where clusters freeze as soon as they reach $L^{\infty}$ diameter at least $N$ for some parameter $N$. We show, informally speaking, that in the limit $N \rightarrow \infty$, the clusters only freeze in the critical window of site percolation on the triangular lattice. Hence the fraction of vertices that eventually (i. e. at time 1) are in a frozen cluster tends to 0 as $N$ goes to infinity. We also show that the diameter of the open cluster at time 1 of a given vertex is, with high probability, smaller than $N$ but of order $N$. This shows that the process on the triangular lattice has a behaviour quite different from Aldous' process. We also indicate which modifications have to be made to adapt the proofs to the case of the $N$-parameter frozen bond percolation process on the square lattice. This extends our results to the square lattice, and answers the questions posed by van den Berg et al. (Random Struct Algorithms 40:220-226, 2012).
\end{abstract}

Keywords Frozen cluster - Critical percolation - Near critical percolation . Correlation length

Mathematics Subject Classification Primary 60K35; Secondary 82B43

\footnotetext{
D. Kiss $(\varangle)$

Statistical Laboratory, Centre for Mathematical Sciences, Cambridge University, Wilberforce Road, Cambridge CB3 0WB, England, UK

e-mail: D.Kiss@statslab.cam.ac.uk

D. Kiss

AIMR, Tohoku University, Sendai, Japan
} 


\section{Contents}

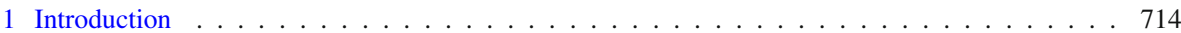

2 Preliminary results on near critical percolation . . . . . . . . . . . . . . . . . . 719

2.1 Correlation inequalities . . . . . . . . . . . . . . . . . . 720

2.2 Mixed arm events, critical arm exponents . . . . . . . . . . . . . . . . . . . . . 720

2.3 Near-critical scaling and correlation length . . . . . . . . . . . . . . . . . . . 724

3 Proof of the main results . . . . . . . . . . . . . . . . . . . . . . . . . . 727

3.1 Proof of Theorem $1.3 \ldots \ldots \ldots \ldots \ldots \ldots \ldots$

3.2 Proof of Corollary $1.7 \ldots \ldots \ldots \ldots 731$

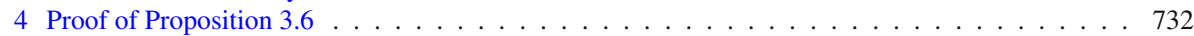

4.1 Notation . . . . . . . . . . . . . . . . . . . . . . 732

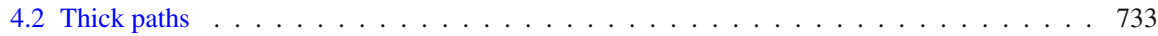

5 Proof of Proposition $3.5 \ldots \ldots \ldots \ldots \ldots \ldots \ldots \ldots \ldots$

5.1 Lowest point of the lowest crossing in parallelograms . . . . . . . . . . . . . . . . 736

5.2 Lowest point of the lowest crossing in regular regions . . . . . . . . . . . . . . . . . . 740

5.3 The diameter of the active clusters close to time $1 / 2 \ldots \ldots \ldots \ldots$. . . . . . . . . 742

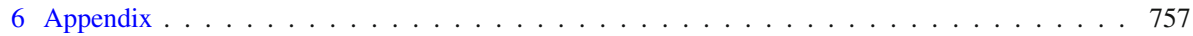

6.1 Winding number of arms . . . . . . . . . . . . . . . . . 757

6.2 Existence of long thick paths in nice regions . . . . . . . . . . . . . . . 761

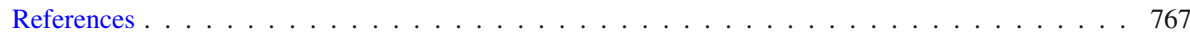

\section{Introduction}

Stochastic processes where small fragments merge and form larger ones are quite useful tools to model physical phenomena at scales ranging from molecular [24] to astronomical ones [29]. The majority of the mathematical literature on such coagulation processes treats mean field models: The rate at which the fragments (clusters) merge is governed only by their sizes-neither the physical location nor their shape affect this rate. See [7] for a review. Stockmayer [24], introduced a mean field model for polymerization where small clusters (sol) merge, however, as soon as a large cluster (gel) forms, it stops growing. In contrast to the mean field models, we consider a model which takes the geometry of the space and the shape of the clusters into account. Following van den Berg et al. [26], and Aldous [4], we introduce the following adaptation of Stockmayer's model. Let $G=(V, E)$ be a graph which represents the underlying geometry and $N \in \mathbb{N}$. For every vertex $v \in V$, independently from each other, we assign a random time $\tau_{v}$ which is uniformly distributed on [0,1]. At time $t=0$, all of the vertices of $G$ are closed. As time increases, a vertex $v$ tries to become open at time $t=\tau_{v}$. It succeeds if and only if all of its neighbours' open clusters (open connected components) at time $t$ have size less than $N$. Note that as soon as the diameter of a cluster reaches $N$, it stops growing, i.e freezes. Hence the name $N$-parameter frozen percolation. Note that we can also consider an edge (bond) version of the model above where edges turn open from closed. This edge version of the process was introduced by van den Berg et al. [26].

We are particularly interested in the $N$-parameter frozen percolation models for large $N$ on graphs such as $d$ dimensional lattices, since they are discrete approximations of the space $\mathbb{R}^{d}$. Herein we restrict to the case where $d=2$. We will mainly work on the triangular lattice. We will see that the behaviour of this model is rich and interesting too, but in a very different way from the model studied by Aldous [4]. 
Let us turn to the model introduced and constructed by Aldous [4]. It is the edge version of the model on the binary tree where we replace the parameter $N$ by $\infty$ in the description above. An edge $e$ of the binary tree opens at time $\tau_{e}$ as long as the open clusters of the endpoints of $e$ are finite. In view of this model, one could also try to construct a similar, so called $\infty$-parameter, model on the triangular lattice. However Benjamini and Schramm [6] showed that it is impossible. Exactly this nonexistence result motivated van den Berg et al. [26] to extend the model of Aldous for finite parameter $N$ : in this case, the $N$-parameter frozen percolation process (both the vertex and the edge version) is a finite range interacting particle system, hence the general theory [18] gives existence. Here we examine how the existence and nonexistence of the $\infty$-parameter models manifest in the $N$-parameter models with large $N$. We concentrate on the two dimensional case specified as follows.

We work on the triangular lattice $\mathbb{T}=(V, E)$ with its usual embedding in the plane $\mathbb{R}^{2}$. That is, the vertex set $V$ is the lattice generated by the vectors $\underline{e}_{1}=(1,0)$ and $\underline{e}_{2}=(\cos (\pi / 3), \sin (\pi / 3))$ :

$$
V:=\left\{a \underline{e}_{1}+b \underline{e}_{2} \mid a, b \in \mathbb{Z}\right\} .
$$

The vertices $u$ and $v$ are neighbours, i.e $(u, v) \in E$ or $u \sim v$ if their $L^{2}$ distance is 1 . We consider the model where we freeze clusters as soon as they reach $L^{\infty}$ diameter (inherited from $\mathbb{R}^{2}$ ) at least $N$. For the case where the underlying lattice is $\mathbb{Z}^{2}$ and for different choices for diameters of clusters see the discussion below Conjecture 1.8.

Van den Berg et al. [28] investigated the edge version of the $N$-parameter process on the binary tree. They found that as $N \rightarrow \infty$, the $N$-parameter process on the binary tree converges to the $\infty$-parameter process in some weak sense. This result raises the question if there is a limit of the $N$-parameter frozen percolation processes on the triangular lattice as $N$ goes to infinity. The non-existence of the $\infty$-parameter process suggests that the $N$-parameter model may have a remarkable (anomalous) behaviour in the limit $N \rightarrow \infty$. It turns out that there is a limiting process, but this process is, in some sense, trivial:

Theorem 1.1 As $N \rightarrow \infty$ the probability that in the $N$-parameter frozen percolation process the open cluster of the origin freezes goes to 0.

To get some intuition for the behaviour of the process, let us for the moment forget about freezing, and call the resulting process the percolation process. That is, at time $\tau_{v}$ the vertex $v$ becomes open no matter how big are the open clusters of its neighbours. Thus at time $t$, a vertex $v$ is open with probability $t$ independently from the other vertices. Hence at time $t$ we see ordinary site percolation with parameter $t$. Recall from [22] that the critical parameter for site percolation on the triangular lattice is $p_{c}=1 / 2$. So at each time $t \leq 1 / 2$ there is no open infinite cluster, and there is a unique infinite open cluster when $t>1 / 2$. Moreover, by [3] at time $t<1 / 2$, the distribution of the size of the open clusters has an exponential decay. Note that if a site is open in the $N$-parameter frozen percolation process at time $t$, then it is also open in the percolation process at time $t$. Hence at time $t<1 / 2$ the $N$-parameter frozen percolation process and the percolation process do not differ too much when $N$ is large: even without freezing, for all $K>0$ the probability that there is an open 
cluster with diameter at least $N$ in a box with side length $K N$ goes to 0 as $N \rightarrow \infty$. To our knowledge, there is no simple argument showing that, roughly speaking, freezing does not take place at times that are essentially bigger than $1 / 2$, which is one of our main results:

Theorem 1.2 For all $K>0$ and $t>1 / 2$, the probability that after time $t$ a frozen cluster forms which intersects a given box with side length $K N$ goes to 0 as $N \rightarrow \infty$.

Compare Theorem 1.2 with $[4,28]$ where it was shown that clusters freeze throughout the time horizon $[1 / 2,1]$ for $N \in \mathbb{N} \cup\{\infty\}$ in the edge version of the $N$-parameter frozen percolation process on the binary tree. (Note that the critical parameter is $1 / 2$ for site percolation on the binary tree.) As it turns out, our method provides a much stronger result than Theorem 1.2. To state it we need some more notation.

Let $\mathbb{P}$ denote the probability measure corresponding to the percolation process. For a fixed $p \in[0,1]$, we call a vertex $v \in V p$-open ( $p$-closed resp.), if its $\tau$ value is less (greater resp.) than $p$. We denote by $\mathbb{P}_{p}$ the distribution of $p$-open vertices.

We borrow some of the notation from [19]. Recall the definition of $V$ from (1.1). The $L^{\infty}$ distance of vertices in $\mathbb{T}$ is the $L^{\infty}$ distance inherited from $\mathbb{R}^{2}$. That is, for $v, w \in V$ the distance $d(v, w)$ between $v=\left(v_{1}, v_{2}\right)$ and $w=\left(w_{1}, w_{2}\right)$ is

$$
\begin{aligned}
d(v, w) & =\|v-w\|_{\infty} \\
& =\max \left\{\left|v_{1}-w_{1}\right|,\left|v_{2}-w_{2}\right|\right\}
\end{aligned}
$$

For $a, b, c, d \in \mathbb{R}$, with $a<b, c<d$ we define the parallelogram

$$
[a, b] \otimes[c, d]:=\left\{k \underline{e}_{1}+l \underline{e}_{2} \mid k \in[a, b] \cap \mathbb{Z}, l \in[c, d] \cap \mathbb{Z}\right\} .
$$

We denote the outer and inner boundary of a set of vertices $S \subseteq V$ by

$$
\begin{aligned}
\partial S & :=\{v \in V \backslash S \mid \exists u \in S: u \sim v\}, \text { and } \\
\partial_{i} S: & =\{v \in S \mid \exists u \in V \backslash S: u \sim v\}
\end{aligned}
$$

respectively. Let $c l(S)=S \cup \partial S$ denote the closure of $S$. For the parallelogram centred around the vertex $v$ with radius $a>0$ we write

$$
B(v ; a):=[-a, a] \otimes[-a, a]+v .
$$

We denote the annulus centred around $v \in V$ with inner radius $a>0$ and outer radius $b>a$ by

$$
A(v ; a, b):=B(v ; b) \backslash B(v ; a) .
$$

We call $B(v ; a)$ the inner, $B(v ; b)$ the outer parallelogram of $A(v ; a, b)$.

We say that there is an open (closed) arm in annulus $A(v ; a, b)$ if there is an open (closed) path from $\partial B(v ; a)$ to $\partial_{i} B(v ; b)$ in $A(v ; a, b)$. We write $o$ for open and $c$ for closed. A colour sequence of length $k$ is an element of $\{o, c\}^{k}$. For $\sigma \in\{o, c\}^{k}$, 
we denote by $\mathcal{A}_{k, \sigma}(v ; a, b)$ the event that there are $k$ disjoint arms in $A(v ; a, b)$ such that the vertices of each of the arms are either all open or all closed, moreover, if we take a counter-clockwise ordering of these arms, then their colours follow a cyclic permutation of $\sigma$.

In the case where $v=\underline{0}=(0,0)$ we omit the first argument in our notation, that is $B(a)=B(\underline{0} ; a)$ etc. We use the notation

$$
\pi_{k, \sigma}(a, b):=\mathbb{P}_{1 / 2}\left(\mathcal{A}_{k, \sigma}(a, b)\right)
$$

for the probability of critical arm events.

In the following we use the near critical parameter scale which was introduced in [13]. For a positive parameter $N$ and $\lambda \in \mathbb{R}$ it is defined as

$$
p_{\lambda}(N):=\frac{1}{2}+\lambda \frac{N^{-2}}{\pi_{4, \text { alt }}(1, N)}
$$

where alt denotes the colour sequence $(o, c, o, c)$.

Before we proceed, let us stop here and let us briefly explain the formula (1.4). Suppose that a vertex $v$ is a closed pivotal vertex, i.e. it is on the boundary of two different open cluster with diameter at least $N$. The two open clusters provide two disjoint open arms starting from neighbouring vertices of $v$. Since the open clusters are different, they have to be separated by closed paths, which provide two disjoint closed arms starting from $v$. Hence the event $\mathcal{A}_{4, \text { alt }}(v ; 1, N)$ occurs. By (1.3), we get that the expected number of pivotal vertices in $B(N)$ is $O\left(N^{2} \pi_{4, \text { alt }}(1, N)\right)$. Let $\lambda>0$. Let us look at the percolation process in the parallelogram $B(N)$ in the time interval $\left[1 / 2, p_{\lambda}(N)\right]$. The probability that a vertex opens in this time interval is $p_{\lambda}(N)-1 / 2$. By a combination of (1.3) and (1.4) we see that the expected number of pivotal vertices which open in this interval is $O(1)$. Hence the parameter scale in (1.4) corresponds to the time scale where open clusters of diameter $O(N)$ merge. See $[13,14]$ for more details.

The considerations above suggest that the parameter scale (1.4) is indeed useful for investigating the $N$-parameter frozen percolation process. We write $\mathbb{P}_{N}$ for the probability measure corresponding to the $N$-parameter frozen percolation process. The following stronger version of Theorem 1.2 is our main result.

Theorem 1.3 For any $\varepsilon, K>0$ there exists $\lambda=\lambda(\varepsilon, K)$ and $N_{0}=N_{0}(\varepsilon, K)$ such that

$\mathbb{P}_{N}$ (a cluster intersecting $B(K N)$ freezes after time $\left.p_{\lambda}(N)\right)<\varepsilon$

for all $N \geq N_{0}$.

In [26] the authors investigated the diameter of the open cluster of the origin at time 1. Their main result is the following.

Definition 1.4 For $t \in[0,1]$ let $C(v ; t)$ denote the open cluster of $v \in V$ at time $t \in[0,1]$. We set $C(t):=C(\underline{0} ; t)$.

Definition 1.5 For $C \subset V$, let $\operatorname{diam}(C)$ denote the $L^{\infty}$-diameter of $C$. 
Theorem 1.6 (Theorem 1.1 of [26]) For the bond version of the $N$-parameter frozen percolation on the square lattice we have

$$
\liminf _{N \rightarrow \infty} \mathbb{P}_{N}(\operatorname{diam}(C(1)) \in(a N, b N))>0
$$

for $a, b \in(0,1)$ with $a<b$.

Analogous result holds for the (site version of) $N$-parameter process on the triangular lattice. In the following corollary we supplement this result. It is an extension of Theorem 1.1.

Corollary 1.7 For any $\varepsilon>0$ there exists $a=a(\varepsilon), b=b(\varepsilon) \in(0,1)$ with $a<b$ and $N_{0}=N_{0}(\varepsilon)$ such that

$$
\mathbb{P}_{N}(\operatorname{diam}(C(1)) \in(a N, b N))>1-\varepsilon
$$

for all $N \geq N_{0}$.

The results above suggest the following intuitive and informal description of the behaviour of $N$-parameter frozen percolation processes on the triangular lattice for large $N$ : At time 0 all the vertices are closed. Then they open independently from each other as in the percolation process till time close to $1 / 2$. Then in the scaling window (1.4), frozen clusters form, and by the end of the window, they give a tiling of $\mathbb{T}$ such that all the holes (non-frozen connected components) have diameter less than $N$ but, typically, of order $N$. After the window, the closed vertices in these holes open as in the percolation process restricted to these holes. At time 1 the non-frozen vertices are all open.

Hence the interesting time scale is (1.4), moreover it raises the question if there is some kind of limiting process which governs the behaviour of the $N$-parameter frozen percolation processes as $N \rightarrow \infty$ in the scaling window (1.4). We have the following, somewhat informal, conjecture:

Conjecture 1.8 When we scale space by $N$ and time according to (1.4), we get a nontrivial scaling limit, which is measurable with respect to the near critical ensemble of $[13,14]$. Moreover, the scaling limit completely describes the frozen clusters of the $N$-parameter frozen percolation as $N \rightarrow \infty$.

Let us mention some generalizations of our results. We considered the site version of the $N$-parameter frozen percolation on the triangular lattice above. We believe that our results hold for bond and site versions of the $N$-parameter frozen percolation models on two dimensional lattices as long as they have sufficient symmetries, e.g invariant under reflection on a line and rotation around a point with some angle $\phi \in(0, \pi)$. We only consider the case of the bond version on the square lattice in detail. See Remark 3.7. Our results remain valid when we use some different distance instead of the $L^{\infty}$ distance in the definition of the $N$-parameter frozen percolation process, as long as the used distance resembles the $L^{\infty}$ distance. Examples of such distances include the $L^{p}$ distances for some $p \geq 1$, or when we rotate the lattice $\mathbb{T}$. Finally let us mention 
that when we freeze clusters when their volumes (number of its vertices) reach $N$, we get a quite different process.

Let us briefly discuss some related results. A version of the $N$-parameter frozen percolation process on $\mathbb{Z}$ and the binary tree were investigated in [9]. We already referred to [4] where Aldous introduced the $\infty$-parameter frozen percolation process on the binary tree. However, we did not mention that this model has another interesting, so called self organized critical (SOC), behaviour: For all $t>1 / 2$, the distribution of the active clusters at time $t$ have the same distribution as critical clusters. Clearly, the $N$-parameter frozen percolation process on the triangular lattice does not have this property. A mean field version of the frozen percolation model on the complete graph was investigated by Ráth in [20]. He showed that this model has similar SOC properties. Let us mention some results on another closely related model, the so called self-destructive percolation. Van den Berg and Brouwer [25] introduced the model and investigated its properties in the cases where the underlying graph is the binary tree and the square lattice $\mathbb{Z}^{2}$. Recently, the model on $\mathbb{Z}^{d}$ for $d=2$ [17], for large $d$ [1] and on non-amenable graphs [2] was investigated. Finally, we refer to [8] where a dynamics similar to frozen percolation was investigated on uniform Cayley trees.

\section{Organization of the paper}

In Sect. 2, we introduce some more notation, and briefly discuss the results from percolation theory required to prove our main result: We start with some classical correlation inequalities in Sect. 2.1. In Sect. 2.2 we introduce mixed arm events where some of the arms can use only the upper half of the annulus, while others can use the whole annulus. Here we also recall some of their well-known properties and discuss some new ones. In particular, we note that the exponent of the arm events increases when we increase the number of arms which have to stay in the upper half plane. The proof of this statement is postponed to Sect. 6.1 of the Appendix. In Sect. 2.3 we describe the connection between the correlation length with the near critical scaling (1.4). We prove Theorem 1.3 and Corollary 1.7 in Sect. 3 assuming two technical results Proposition 3.5 and 3.6. In Sect. 4 we introduce some more notation and the notion of thick paths. There we prove Proposition 3.6. In this proof a deterministic (combinatorial/geometric) result, Lemma 4.5, plays an important role. The proof of this lemma is postponed to Sect. 6.2 of the Appendix. The most technical part of the paper is Sect. 5 where we prove Proposition 3.5. In Sects. 5.1 and 5.2 we investigate the vertical position of the lowest point of the lowest closed crossing in regions with half open half closed boundary conditions. We combine these results with the ones in Sect. 2 and conclude the proof of Proposition 3.5 in Sect. 5.3. This finishes the proof of the main result.

\section{Preliminary results on near critical percolation}

We recall some classical results from percolation theory in this section. With suitable modifications, the results of this section also hold for bond percolation on the square lattice unless it is indicated otherwise. 


\subsection{Correlation inequalities}

We use the following two inequalities throughout the paper. See Section 2.2 and 2.3 of [15] for more details.

Definition 2.1 Let $A \subset\{o, c\}^{V}$ and $U \subseteq V$. We say that an event $A \subset\{o, c\}^{V}$ is increasing in the configuration in $U$, if for all $\omega \in A$ we have $\omega^{\prime} \in A$ where

$$
\omega_{v}^{\prime}= \begin{cases}\omega_{v} \text { or } o & \text { for } v \in U \\ \omega_{v} & \text { for } v \in V \backslash U .\end{cases}
$$

That is, turning some closed vertices in $U$ into open ones can only help the occurrence of $A$. We say that $A$ is decreasing in the configuration in $U$, if its complement is increasing in the configuration in $U$. In the case where $U=V$ we simply say that $A$ is an increasing/decreasing event.

The first inequality is due to Fortuin et al. [12]:

Theorem 2.2 (FKG) For any pair of increasing events $A, B$ we have

$$
\mathbb{P}_{p}(A \cap B) \geq \mathbb{P}_{p}(A) \mathbb{P}_{p}(B) .
$$

The second is due to van den Berg and Kesten [27] for an extension for general events see [21].

Theorem 2.3 (BK) Let $A, B$ be increasing events, then

$$
\mathbb{P}_{p}(A \square B) \leq \mathbb{P}_{p}(A) \mathbb{P}_{p}(B),
$$

where $A \square B$ denotes the disjoint occurrence of the events $A$ and $B$.

\subsection{Mixed arm events, critical arm exponents}

Recall the definition of arm events from the introduction. There the arms were allowed to use the whole annulus. We introduce the mixed arm events, where some of the arms lie in the upper half of the annulus, while others can use the whole annulus:

Definition 2.4 Let $l, k \in \mathbb{N}$ with $0 \leq l \leq k$, and a colour sequence $\sigma$ of length $k$. Let $v \in V$ and $a, b \in(1, \infty)$ with $a<b$. The full plane $k, l$ mixed arm event with colour sequence $\sigma$ in the annulus $A(v ; a, b)$ is denoted by $\mathcal{A}_{k, l, \sigma}(v ; a, b)$. It is the normal $k$ arm event $\mathcal{A}_{k, \sigma}(v ; a, b)$ of the Introduction with the extra condition that there is a counter-clockwise ordering of the arms such that the colour of the arms follow $\sigma$, and the first $l$ arms lie in the half annulus $A(v ; a, b) \cap(\mathbb{Z} \otimes[0, \infty)+v)$. When $v=0$, we omit the first argument from these notations.

We extend the definition (1.3) for mixed arm events by defining

$$
\pi_{k, l, \sigma}(a, b):=\mathbb{P}_{1 / 2}\left(\mathcal{A}_{k, l, \sigma}(a, b)\right) \text {. }
$$


Remark 2.5 In the case $k=l$, we get the so called half plane arm events.

We fix $n_{0}(k)=10 k$ for $k \in \mathbb{N}$. Note that the event $\mathcal{A}_{k, l, \sigma}(n, N)$ is non-empty whenever $n_{0}(k)<n<N$. Let us summarize the known critical arm exponents for site percolation on the triangular lattice. To our knowledge, Theorem 2.6 in its generality is not known to hold for bond percolation on $\mathbb{Z}^{2}$.

Theorem 2.6 (Theorem 3 and 4 of [23]) Let $l, k \in \mathbb{N}$ and $\sigma$ be a colour sequence of length $k$. We define $a_{k, l}(\sigma)$

- for $k=1, l=0$ and any colour sequence $\sigma$ as

$$
\alpha_{1,0}(\sigma):=\frac{5}{48}
$$

- for $k>1$ and $l=0$, when $\sigma$ contains both colours, as

$$
\alpha_{k, 0}(\sigma):=\frac{k^{2}-1}{12}
$$

- for $k=l \geq 1$ and any colour sequence $\sigma$ as

$$
\alpha_{k, k}(\sigma):=\frac{k(k+1)}{6} .
$$

In these cases we have

$$
\pi_{k, l, \sigma}\left(n_{0}(k), N\right)=N^{-\alpha_{k, l}(\sigma)+o(1)}
$$

as $N \rightarrow \infty$,

To our knowledge, for general $k$ and $l$, neither the value, nor the existence of the exponents is known. We expect that the exponents do exist. We will see in Proposition 6.5, that if $\alpha_{k, l}(\sigma)$ and $\alpha_{k, m}(\sigma)$ exists for some $k, l, m \in \mathbb{N}$ and $\sigma \in\{o, c\}^{k}$ with $m<l$, then $\alpha_{k, m}(\sigma)<\alpha_{k, l}(\sigma)$. Since we do not need such general result, we only prove the following proposition in detail.

Proposition 2.7 For any $k \geq 1$, there are positive constants $c=c(k), \varepsilon=\varepsilon(k)$ such that

$$
\pi_{k, l, \sigma}\left(n_{0}(k), N\right) \leq c N^{-\varepsilon} \pi_{k, 0, \sigma}\left(n_{0}(k), N\right)
$$

for $l=1,2, \ldots, k$ uniformly in $N$ and in the colour sequence $\sigma$.

Remarks 2.8 1. We do not need the exact values of the critical exponents of Theorem 2.6. For our purposes it is enough to show that certain arm events have exponents at least 2 .

2. Proposition 2.7 and its generalization also hold for mixed arm events in bond percolation on the square lattice. 
Proof of Proposition 2.7 Proposition 2.7 is a simple corollary of Proposition 6.3 of the Appendix. Loosely speaking, it states that conditioning on the event that we have $k$ arms in $A(a, b)$, these arms wind around the origin in $c \log (b / a)$ disjoint sub-annuli of $A(a, b)$ for some small but fixed positive constant $c$ with probability at least $1-\left(\frac{a}{b}\right)^{\kappa}$ for some $\kappa>0$. The proof of Proposition 6.3 can be found in the Appendix.

Remark 2.9 Recall that we do not know in general if the exponents $\alpha_{k, l}(\sigma)$ exist or not. Nonetheless, on the triangular lattice, Proposition 2.7 and Theorem 2.6 and the BK inequality (Theorem 2.3) give that for any colour sequence $\sigma$, there is an upper bound with exponent strictly larger than 2 for $\pi_{k, l, \sigma}\left(n_{0}(k), N\right)$ when

- $k \geq 6$, and $l \geq 0$, or

- $k \geq 5$ and $l \geq 1$, or

- $k \geq 4$ and $l \geq 3$.

For arm events with exponents larger than 2 in the case of bond percolation on the square lattice see Remark 2.14 below.

Another well-known attribute of critical arm events is their quasi-multiplicative property. For the full plane, respectively for half plane, arm events this property is shown to hold in Proposition 17 of [19], respectively in Section 4.6 of [19]. Simple modifications of these arguments apply to mixed arm events. We introduce the notation $\asymp$ when the ratio of the two quantities is bounded away from 0 and $\infty$. We have:

Proposition 2.10 Let $k \geq 1$ and $\sigma \in\{o, c\}^{k}$. Then

$$
\pi_{k, l, \sigma}\left(n_{1}, n_{2}\right) \pi_{k, l, \sigma}\left(n_{2}, n_{3}\right) \asymp \pi_{k, l, \sigma}\left(n_{1}, n_{3}\right)
$$

uniformly in $n_{0}(k) \leq n_{1} \leq n_{2} \leq n_{3}$.

In the following lemma we consider arm events where the open arms are $p$-open and the closed arms are $q$-closed where $p, q \in[0,1]$ with $p$ not necessarily equal to $q$. When $p$ and $q$ are of the form (1.4), then we call these arm events near critical arm events. In this case the probabilities of these events are comparable to critical arm event probabilities. The following lemma is a generalization of Lemma 8.4 [14] and Lemma 6.3 of [10].

Lemma 2.11 Let $v \in V, \lambda_{1}, \lambda_{2} \in \mathbb{R}$ and $a, b \in(0,1)$ with $a<b$. Let $\mathcal{A}_{k, l, \sigma}^{\lambda_{1}, \lambda_{2}, N}(v ; a N, b N)$ denote the modification of the event $\mathcal{A}_{k, l, \sigma}(v ; a N, b N)$ where the open arms are $p_{\lambda_{2}}(N)$-open and the closed arms are $p_{\lambda_{1}}(N)$-closed. Then there are positive constants $c=c\left(\lambda_{1}, \lambda_{2}, k\right)$ and $N_{0}=N_{0}\left(\lambda_{1}, \lambda_{2}, a, b, k\right)$ such that

$$
\mathbb{P}\left(\mathcal{A}_{k, l, \sigma}^{\lambda_{1}, \lambda_{2}, N}(v ; a N, b N)\right) \leq c \pi_{k, l, \sigma}(a N, b N)
$$

for $N \geq N_{0}$.

Proof of Lemma 2.11 It follows from either of the proof of Lemma 8.4 of [14] or from the proof of Lemma 6.3 of [10]. 
In the following events we collect some of the near critical arm events which have upper bounds with exponents strictly larger than 2 . These events play a crucial role in our main result.

Definition 2.12 Let $a, b \in(0,1), \lambda_{1}, \lambda_{2} \in \mathbb{R}, K>0$ and $N \in \mathbb{N}$ with $a<b$. Recall that the event $\mathcal{A}_{k, l, \sigma}^{\lambda_{1}, \lambda_{2}, N}(v ; a N, b N)$, roughly speaking, is defined as having $k$ arms in a certain annulus, with the 'first' $l$ arms stay in the upper half plane with boundary passing through $v$. One can also define events where these first $l$ arms utilise the lower, left or right half plane with boundary passing through $v$.

Let $\mathcal{N} \mathcal{A}^{c}\left(a, b, \lambda_{1}, \lambda_{2}, K, N\right)$ denote the union of the events $\mathcal{A}_{k, l, \sigma}^{\lambda_{1}, \lambda_{2}, N}(v ; a N, b N)$ for $(k, l) \in\{(4,3),(5,1),(6,0)\}, \sigma \in\{o, c\}^{k}, v \in B(K N)$ as well as the versions of these events where the first $l$ arms can only use the lower, left or right half of the annulus $A(v ; a N, b N)$. We define $\mathcal{N} \mathcal{A}\left(a, b, \lambda_{1}, \lambda_{2}, K, N\right)$ as the complement of the event above.

We show that for fixed $b, K, \lambda_{1}$ and $\lambda_{2}$, we can set $a \in(0,1)$ so that the probability of $\mathcal{N} \mathcal{A}\left(a, b, \lambda_{1}, \lambda_{2}, K, N\right)$ becomes as close to 1 as we require for large $N$. More precisely, we prove the following:

Corollary 2.13 There is $\tilde{\varepsilon}>0$ such that for all $a, b \in(0,1)$, with $a<b$ and $\lambda_{1}, \lambda_{2} \in$ $\mathbb{R}$ there are positive constants $c=c\left(\lambda_{1}, \lambda_{2}, K\right)$ and $N_{0}=N_{0}\left(a, b, \lambda_{1}, \lambda_{2}, K\right)$ such that

$$
\mathbb{P}\left(\mathcal{N} \mathcal{A}\left(a, b, \lambda_{1}, \lambda_{2}, K, N\right)\right) \geq 1-c \frac{a^{\tilde{\varepsilon}}}{b^{2+\tilde{\varepsilon}}}
$$

for $N \geq N_{0}$.

Proof of Corollary 2.13 Suppose that one of the arm events in Definition 2.12, for example $\mathcal{A}_{k, l, \sigma}^{\lambda_{1}, \lambda_{2}, N}(v ; a N, b N)$ for some $v \in B(K N)$, occurs. Then the event $\mathcal{A}_{k, l, \sigma}^{\lambda_{1}, \lambda_{2}, N}\left(\lfloor 2 a N\rfloor z ; 2 a N, \frac{b}{2} N\right)$ occurs for some $z \in V$ with $z \in B\left(\left\lceil\frac{a+K}{2 a}\right\rceil\right)$.

Combination of Remark 2.9 and Lemma 2.11 gives that there are constants $c^{\prime}=$ $c^{\prime}\left(\lambda_{1}, \lambda_{2}\right), N_{0}=N_{0}\left(a, b, \lambda_{1}, \lambda_{2}\right)$, and a universal constant $\tilde{\varepsilon}>0$ such that the probability of one of these events is at most

$$
c^{\prime}\left(\frac{2 a}{b / 2}\right)^{2+\varepsilon}
$$

for $N \geq N_{0}$. The same argument works for other arm events which appear in Definition 2.12, and provide an upper bound similar to (2.2). Hence (2.2) combined with $\left|B\left(\left\lceil\frac{a+K}{2 a}\right\rceil\right)\right|=O\left(a^{-2}\right)$ concludes the proof of Corollary 2.13.

Remark 2.14 To our knowledge it is not known if the direct analogue of Corollary 2.13 holds on the square lattice. The reason is that the exponent $\alpha_{5,0}(\sigma)$ and $\alpha_{3,3}(\sigma)$ is not known for general $\sigma$. This is due to that the colour switching argument (Proposition 19. of [19]) cannot be transferred to bond percolation for the square lattice, or at least not without additional considerations. 
We recall the proof of Theorem 24 and Remark 26 of [19], where it is shown that $\alpha_{5,0}(o, c, o, o, c)=2$ and $\alpha_{3,3}(c, o, c)=2$ for lattices with sufficient symmetries, in particular for the square lattice. This implies that a version of Corollary 2.13 holds for the square lattice if we modify Definition 2.12 so that we only forbid the occurrence of those arm events where the required set of arms contain

- three half plane arm events with colour sequence $(o, c, o)$ or $(c, o, c)$, or

- five full plane arms with colour sequence $(o, c, o, o, c)$ or $(c, o, c, c, o)$

as a subset. See Remark 26 of [19].

\subsection{Near-critical scaling and correlation length}

Recall that in Sect. 1 we already gave an explanation for the near critical parameter scale (1.4). In this section we give a different interpretation of this parameter scale, which is connected to the correlation length introduced by Kesten in [16].

We say that there is an open (closed) horizontal crossing of a parallelogram $B:=$ $[a, b] \otimes[c, d]$ if there is an open (closed) path connecting $\{\lceil a\rceil\} \otimes[c, d]$ and $\{\lfloor b\rfloor\} \otimes$ $[c, d]$ in $[a, b] \otimes[c, d]$. For the event that there is an open (closed, resp.) horizontal crossing of $B$ we use the notation $\mathcal{H}_{o}(B)\left(\mathcal{H}_{c}(B)\right.$, resp.). One can define similar events for vertical crossings, which we denote by $\mathcal{V}_{o}(B)$ and $\mathcal{V}_{c}(B)$ respectively. For $\varepsilon \in(0,1 / 2)$ the correlation length is defined as

$$
L_{\varepsilon}(p)= \begin{cases}\min \left\{n \mid \mathbb{P}_{p}\left(\mathcal{H}_{o}(B(n))\right) \leq \varepsilon\right\} & \text { when } p<p_{c} \\ \min \left\{n \mid \mathbb{P}_{p}\left(\mathcal{H}_{o}(B(n))\right) \geq 1-\varepsilon\right\} & \text { when } p>p_{c}\end{cases}
$$

Remark 2.15 The particular choice of $\varepsilon$ is not important in this definition. Indeed, Corollary 37 of [19], or alternatively Corollary 2 of [16], gives that

$$
L_{\varepsilon}(p) \asymp L_{\varepsilon^{\prime}}(p)
$$

for any $\varepsilon, \varepsilon^{\prime} \in(0,1 / 2)$ uniformly in $p \in(0,1)$.

We show that the control over the near critical parameter $\lambda$ gives a control over the correlation length in Corollary 2.17 and 2.18 below. Recall the remark after Lemma 8 of [16]:

Proposition 2.16 For any fixed $\varepsilon \in(0,1 / 2)$, we have

$$
\left|p-p_{c}\right|\left(L_{\varepsilon}(p)\right)^{2} \pi_{4,0, a l t}\left(1, L_{\varepsilon}(p)\right) \asymp 1
$$

uniformly for $p \neq 1 / 2$.

Note that for fixed $\varepsilon>0$, the correlation length $L_{\varepsilon}(p)$ is a decreasing (increasing, resp.) function of $p$ for $p>p_{c}\left(p<p_{c}\right.$, resp.). By combining this and Proposition 2.10 we get:

Corollary 2.17 For all $\lambda \in \mathbb{R} \backslash\{0\}$ and $\varepsilon \in(0,1 / 2)$,

$$
L_{\varepsilon}\left(p_{\lambda}(N)\right) \asymp N \text {. }
$$


Corollary 2.18 For any $C>0$ and $\varepsilon \in(0,1 / 2)$ there exits $\lambda_{1}=\lambda_{1}(C, \varepsilon)>0$ and $N_{1}=N_{1}(C, \varepsilon)$ such that for any $\lambda \in \mathbb{R}$ with $|\lambda| \geq \lambda_{1}$ we have

$$
L_{\varepsilon}\left(p_{\lambda}(N)\right) \leq C N
$$

for $N \geq N_{1}$. Also, for any $c>0$, and $\varepsilon \in(0,1 / 2)$ there exists $\lambda_{2}(c, \varepsilon)>0$ and $N_{2}=N_{2}(c, \varepsilon)$ such that for any $\lambda \in \mathbb{R} \backslash\{0\}$ with $|\lambda| \leq \lambda_{2}$ we have

$$
L_{\varepsilon}\left(p_{\lambda}(N)\right) \geq c N
$$

for $N \geq N_{2}$

Remark 2.19 On the triangular lattice, a ratio limit theorem for $\pi_{4,0, \text { alt }}$, Proposition 4.7 of [13] holds. This combined with the definition of $L_{\varepsilon}(p)$, and Proposition 2.16 shows that the following stronger statement holds on the triangular lattice. See also Theorem 10.7 of [14].

Claim For all $\lambda_{1}, \lambda_{2} \in \mathbb{R}$ with $\lambda_{1} \leq \lambda_{2}, \lambda_{1} \lambda_{2}>0$ and $\varepsilon \in(0,1 / 2)$ there are positive constants $c=c(\varepsilon), C=C(\varepsilon)$ and $N_{0}=N_{0}\left(\varepsilon, \lambda_{1}, \lambda_{2}\right)$ such that

$$
c N|\lambda|^{-4 / 3} \leq L_{\varepsilon}\left(p_{\lambda}(N)\right) \leq C N|\lambda|^{-4 / 3}
$$

for all $\lambda \in\left[\lambda_{1}, \lambda_{2}\right]$ and $N \geq N_{0}$.

Standard Russo-Seymour-Welsh (RSW) techniques and the definition of the correlation length give that the control over the correlation length gives a control over the crossing probabilities of parallelograms. This combined with the two corollaries above show that the control over the near critical parameter gives control over the crossing probabilities. See Corollary 2.20 and 2.21 below:

Corollary 2.20 For all $\lambda \in \mathbb{R}$ and $a, b \in(0, \infty)$, there are constants $c=c(a, b, \lambda) \in$ $(0,1), C=C(a, b, \lambda) \in(0,1)$ and $N_{0}=N_{0}(a, b, \lambda)$ such that

$$
\begin{aligned}
& c<\mathbb{P}_{p_{\lambda}(N)}\left(\mathcal{H}_{o}([0, a N] \otimes[0, b N])\right)<C \\
& c<\mathbb{P}_{p_{\lambda}(N)}\left(\mathcal{H}_{c}([0, a N] \bigotimes[0, b N])\right)<C
\end{aligned}
$$

for $N \geq N_{0}$.

Corollary 2.21 Let $\delta \in(0,1)$, and $a, b \in(0, \infty)$. There exists $\lambda_{1}=\lambda_{1}(\delta, a, b)>0$ and $N_{1}=N_{1}(\delta, a, b)$ such that for all $\lambda \geq \lambda_{1}$

$$
\mathbb{P}_{p_{\lambda}(N)}\left(\mathcal{H}_{o}([0, a N] \otimes[0, b N])\right)>1-\delta
$$

for $N \geq N_{1}$. Furthermore, there exists $\lambda_{2}=\lambda_{2}(\delta, a, b)<0$ and $N_{2}=N_{2}(\delta, a, b)$ such that for all $\lambda \leq \lambda_{2}$

$$
\mathbb{P}_{p_{\lambda}(N)}\left(\mathcal{H}_{c}([0, a N] \otimes[0, b N])\right)>1-\delta
$$

for $N \geq N_{2}$. 
Similar RSW techniques show that it is unlikely to have crossing in a thin and long parallelogram in the hard direction in the critical window. See Remark 40 [19] for more details.

Corollary 2.22 Let $\lambda \in \mathbb{R}$, and $a, b \in(0,1)$. There exist positive constants $c=c(\lambda)$, $C=C(\lambda)$ and $N_{0}=N_{0}(\lambda, a, b)$ such that

$$
\mathbb{P}_{p_{\lambda}(N)}\left(\mathcal{H}_{o}([0, a N] \bigotimes[0, b N])\right) \leq C \exp \left(-c \frac{a}{b}\right)
$$

for $N \geq N_{0}$.

The following event plays a crucial role in the proof of our main result.

Definition 2.23 Let $a, b \in(0,1), \lambda_{1}, \lambda_{2} \in \mathbb{R}$, and $N \in \mathbb{N}$ with $a<b$. Let $\mathcal{N C}\left(a, b, \lambda_{1}, \lambda_{2}, K, N\right)$ denote the event that for all parallelograms $B=[0, a N] \otimes$ $[0, b N]+z$ with $z \in B(K N)$, there is neither a $p_{\lambda_{1}}(N)$-open nor a $p_{\lambda_{2}}(N)$-closed horizontal crossing in $B$.

The following Corollary 2.24 follows from Corollary 2.22 by arguments analogous to the proof of Corollary 2.13.

Corollary 2.24 Let $a, b \in(0,1), \lambda_{1}, \lambda_{2} \in \mathbb{R}$, and $N \in \mathbb{N}$ with $a<b$. There are positive constants $c=c\left(\lambda_{1}, \lambda_{2}\right), C=C\left(\lambda_{1}, \lambda_{2}\right)$ and $N_{0}=N_{0}\left(a, b, \lambda_{1}, \lambda_{2}\right)$ such that

$$
\mathbb{P}\left(\mathcal{N C}\left(a, b, \lambda_{1}, \lambda_{2}, K, N\right)\right) \geq 1-C a^{-2} \exp \left(-c \frac{b}{a}\right)
$$

for $N \geq N_{0}$.

We finish this section by stating two lemmas which will be used explicitly in the proof of our main result.

Lemma 2.25 Let $\lambda \in \mathbb{R}, a, b \in(0, \infty)$ and $\varepsilon>0$. Then there are positive integers $k=k(\lambda, a, b, \varepsilon)$ and $N_{0}=N_{0}(\lambda, a, b, \varepsilon)$ such that

$\mathbb{P}_{p_{\lambda}(N)}$ (there are at least $k$ disjoint closed arms in $\left.A(a N, b N)\right)<\varepsilon$

for $N \geq N_{0}$.

Proof of Lemma 2.25 This is a consequence of Corollary 2.20 and the BK inequality (Theorem 2.3). The proof also appears in the proof of Lemma 15 of [19].

Definition 2.26 Let $a, b, c, d, f \in \mathbb{R}$ with $a \leq b, c \leq d$ and $f>0$. We say that there is an open (closed) $f$-net in $B=[a, b] \otimes[c, d]$ if there is an open (closed) vertical crossing in the parallelograms $[a+i\lfloor f\rfloor, a+(i+1)\lfloor f\rfloor-1] \bigotimes$ $[c, d]$, and there is an open (closed) horizontal crossing in the parallelograms $[a, b] \otimes[c+j\lfloor f\rfloor, c+(j+1)\lfloor f\rfloor-1]$ for $i=0,1, \ldots,\lfloor(b-a) /\lfloor f\rfloor\rfloor$ and $j=0,1, \ldots,\lfloor(d-c) /\lfloor f\rfloor\rfloor$.

For $\lambda \in \mathbb{R}$ and $\delta \in(0, \infty), \mathcal{N}_{c}(\lambda, \delta, K, N)\left(\mathcal{N}_{o}(\lambda, \delta, K, N)\right.$, resp. ) denotes the event that there is a $p_{\lambda}(N)$-closed $\left(p_{\lambda}(N)\right.$-open, resp.) $\delta N$-net in $B(K N)$. 
Lemma 2.27 Let $\varepsilon, \delta, K>0$. There exists $\lambda_{1}=\lambda_{1}(\varepsilon, \delta, K) \in \mathbb{R}$ and $N_{1}=$ $N_{1}(\varepsilon, \delta, K)$ such that

$$
\mathbb{P}\left(\mathcal{N}_{o}\left(\lambda_{1}, \delta, K, N\right)\right)>1-\varepsilon
$$

for $N \geq N_{1}$. Moreover there exist $\lambda_{2}=\lambda_{2}(\varepsilon, \delta, K) \in \mathbb{R}$ and $N_{2}=N_{2}(\varepsilon, \delta, K)$ such that

$$
\mathbb{P}\left(\mathcal{N}_{c}\left(\lambda_{2}, \delta, K, N\right)\right)>1-\varepsilon
$$

for $N \geq N_{2}$.

Proof of Lemma 2.27 This is a consequence of Corollary 2.21 and the FKG inequality (Theorem 2.2).

\section{Proof of the main results}

We prove our main results Theorem 1.3 and Corollary 1.7 in this section assuming Proposition 3.5 and 3.6.

Definition 3.1 In the $N$-parameter frozen percolation process we call a vertex frozen at some time $t \in[0,1]$, if either it or one of its neighbours have an open cluster with diameter bigger than $N$ at time $t$. If a site is not frozen at time $t$, then we say it is active at time $t$. Note that both frozen and active sites can be open or closed. We say that $F$ is a (open) frozen cluster at time $t \in[0,1]$ if it is a connected component of the open vertices at time $t$ with $\operatorname{diam}(F) \geq N$. In the case where $t=1$, we simply say that $F$ is a frozen cluster.

Recall Definition 2.26. We observe the following.

Observation 3.2 Let $K>0$ and $N \in \mathbb{N}$. Then in the $N$-parameter frozen percolation process there is no frozen cluster at time $p_{\lambda}(N)$ in $B(K N)$ on the event $\mathcal{N}_{c}(\lambda, 1 / 6, K+2, N)$. Hence on $\mathcal{N}_{c}(\lambda, 1 / 6, K+2, N)$, a vertex in $B(K N)$ is open (closed, resp.) in the $N$-parameter frozen percolation process at time $p_{\lambda}(N)$ if and only if it is $p_{\lambda}(N)$-open $\left(p_{\lambda}(N)\right.$-closed, resp.).

We show that the number of frozen clusters intersecting $B(K N)$ in the $N$-parameter frozen percolation process is tight in $N$.

Lemma 3.3 Let $K>0$ and $N \in \mathbb{N}$. Let $F C(t, K, N)$ denote the number of frozen clusters intersecting $B(K N)$ at time $t \in[0,1]$ in the $N$-parameter frozen percolation process. Then for all $\varepsilon>0$ there exists $L=L(\varepsilon, K)$ and $N_{0}=N_{0}(\varepsilon, K)$ such that

$$
\mathbb{P}_{N}(F C(1, K, N)>L)<\varepsilon
$$

for $N \geq N_{0}$. 
Proof of Lemma 3.3 By Lemma 2.27 we set $\lambda=\lambda(\varepsilon, K) \in \mathbb{R}$ such that

$$
\mathbb{P}_{N}\left(\mathcal{N}_{c}(\lambda, 1 / 6, K+4, N)\right)>1-\frac{1}{2} \varepsilon
$$

for $N \geq N_{1}(\varepsilon, K)$. Let $F$ be an open frozen cluster which intersects $B(K N)$. From Observation 3.2 we get the vertices of $\partial F$ are closed at $p_{\lambda}(N)$ in the $N$-parameter percolation process on the event $\mathcal{N}_{c}(\lambda, 1 / 6, K+4, N)$.

Let us cover the parallelogram $B(K N)$ with the annuli

$$
A_{z}=A(\lfloor N / 20\rfloor z ;\lfloor N / 20\rfloor,\lfloor N / 10\rfloor) \text { with } z \in B(\lceil 20 K\rceil) .
$$

Suppose that there is an open frozen cluster in the $N$-parameter frozen percolation which has a vertex in $B(K N)$. The construction of the annuli above gives that there is $z \in B(\lceil 20 K\rceil)$ such that $B(\lfloor N / 20\rfloor z ;\lfloor N / 20\rfloor)$, the inner parallelogram of $A_{z}$, contains a vertex of this open frozen cluster. Since the diameter of $B(\lfloor N / 20\rfloor z ;\lfloor N / 10\rfloor)$ is less than $N$, this cluster has to cross the annulus $A_{z}$. Hence for each open frozen cluster intersecting $B(K N)$, we find at least one open frozen crossing of an annulus $A_{z}$. Moreover, if there are $k \geq 2$ different frozen clusters crossing the annulus $A_{z}$, then there are at least $k$ disjoint closed frozen arms which separate the open frozen clusters in $A_{z}$ at time 1 . By the arguments above, these arms are $p_{\lambda}(N)$-closed. Thus the number of different frozen clusters intersecting $B(\lfloor N / 20\rfloor z ;\lfloor N / 20\rfloor)$ is bounded above by $1 \vee l_{z}$, where $l_{z}$ is the number of disjoint $p_{\lambda}(N)$-closed arms of $A_{z}$. Hence by the translation variance of the $N$-parameter frozen percolation process we have

$$
\begin{aligned}
& \mathbb{P}_{N}\left(F C(1, K, N) \geq L, \mathcal{N}_{c}(\lambda, 1 / 24)\right) \leq \mathbb{P}_{p_{\lambda}(N)}\left(\sum_{z \in B(\lceil 20 K\rceil)}\left(1 \vee l_{z}\right) \geq L\right) \\
& \quad \leq \mathbb{P}_{p_{\lambda}(N)}\left(\exists z \in B(\lceil 20 K\rceil) \text { such that } l_{z} \geq(2\lceil 20 K\rceil+1)^{-2} L\right) \\
& \quad \leq(2\lceil 20 K\rceil+1)^{2} \mathbb{P}_{p_{\lambda}(N)}\left(l_{0} \geq(2\lceil 20 K\rceil+1)^{-2} L\right) .
\end{aligned}
$$

By Lemma 2.25 we set $L=L(\varepsilon, K) \geq(2\lceil 20 K\rceil+1)^{2}$ and $N_{2}=N_{2}(\varepsilon, K)$ such that

$$
\mathbb{P}_{p_{\lambda}(N)}\left(l_{0} \geq L / 200^{2}\right)<\frac{1}{2}(2\lceil 20 K\rceil+1)^{-2} \varepsilon
$$

for $N \geq N_{2}$. This combined with (3.2) gives that

$$
\mathbb{P}_{N}\left(F C(1, K, N) \geq L, \mathcal{N}_{c}(\lambda, 1 / 6, K+4, N)\right)<\frac{1}{2} \varepsilon
$$

for $N \geq N_{2}$. We set $N_{0}:=N_{1} \vee N_{2}$. A combination of (3.1) and (3.3) finishes the proof of Lemma 3.3. 
Definition 3.4 For $v \in V$ and $\lambda \in \mathbb{R}$ let $\mathcal{C}_{a}(v ; \lambda)=\mathcal{C}_{a}(v ; \lambda, N)$ denote the active cluster of $v$ in the $N$-parameter frozen percolation process at time $p_{\lambda}(N)$. We omit the first argument from the notation above when $v=\underline{0}$.

We state the two propositions below which play a crucial role in the proof of Theorem 1.3. The proofs of these propositions are rather technical, so we postpone them to the next section. The first proposition shows that for $\alpha>0$, it is unlikely to have an active cluster at time $p_{\lambda}(N)$ which intersects $B(K N)$ and has diameter close to $\alpha N$.

Proposition 3.5 For all $\lambda \in \mathbb{R}$ and $\varepsilon, K, \alpha>0$, there exist $\theta=\theta(\lambda, \alpha, \varepsilon, K) \in$ $(0,1 / 2)$ and $N_{0}=N_{0}(\lambda, \alpha, \varepsilon, K)$ such that

$$
\mathbb{P}_{N}\left(\exists v \in B(K N) \text { s.t. } \operatorname{diam}\left(\mathcal{C}_{a}(v ; \lambda)\right) \in((\alpha-\theta) N,(\alpha+\theta) N)\right)<\varepsilon
$$

for $N \geq N_{0}$.

The second proposition claims that if there is a vertex $v \operatorname{such}$ that $\operatorname{diam}\left(\mathcal{C}_{a}\left(v ; \lambda_{1}, N\right)\right)$ $\geq(1+\theta) N$ then some part of $\mathcal{C}_{a}\left(v ; \lambda_{1}, N\right)$ freezes 'soon':

Proposition 3.6 Let $\theta \in(0,1), \varepsilon>0$ and $\lambda_{1}, K, \in \mathbb{R}$. Recall the notation $F C(t, K+2, N)$ from Lemma 3.3. There exists $\lambda_{2}=\lambda_{2}\left(\lambda_{1}, \theta, \varepsilon\right)$ and $N_{0}=$ $N_{0}\left(\lambda_{1}, \theta, \varepsilon\right)$ such that the probability of the intersection of the events

- $\exists v \in B(K N)$ such that $\operatorname{diam}\left(\mathcal{C}_{a}\left(v ; \lambda_{1}, N\right)\right) \geq(1+\theta) N$, and

- none of the clusters intersecting $B((K+2) N)$ freeze in the time interval $\left(p_{\lambda_{1}}(N), p_{\lambda_{2}}(N)\right]$, i.e.

$$
F C\left(p_{\lambda_{1}}(N), K+2, N\right)=F C\left(p_{\lambda_{2}}(N), K+2, N\right)
$$

is less than $\varepsilon$ for $N \geq N_{0}$.

Before we turn to the proof of our main results we make a remark on how to adapt the proofs for the $N$-parameter frozen bond percolation process on the square lattice.

Remark 3.7 The arguments in Sect. 3, 4, and 5 and in the Appendix can be easily adapted to the $N$-parameter frozen bond percolation on the square lattice. Some care is required when we use Corollary 2.13: As we already noted in Remark 2.14, the direct analogue of Corollary 2.13 does not hold on the square lattice. However, one can check that the version of Corollary 2.13 which was proposed in Remark 2.14 is enough for the proofs appearing in Sects. 3, 4, and 5.

\subsection{Proof of Theorem 1.3}

Proof of Theorem 1.3 The proof follows the following informal strategy. Consider the following procedure. We set $\lambda_{1}=0$. We look at the $N$-parameter percolation process at time $p_{\lambda_{1}}(N)$. We have two cases. 
In the first case all the active clusters at time $p_{\lambda_{1}}(N)$ intersecting $B(K N)$ have diameter less than $N$. Hence no cluster intersecting $B(K N)$ can freeze after $p_{\lambda_{1}}(N)$. We terminate the procedure.

In the second case there is $v \in B(K N)$ such that the active cluster $\mathcal{C}_{a}\left(v ; \lambda_{1}, N\right)$ has diameter at least $N$. Using Proposition 3.5 we $\operatorname{set} \theta_{1}$ such that the diameter of this cluster is at least $\left(1+\theta_{1}\right) N$ with probability close to 1 . If $\operatorname{diam}\left(\mathcal{C}_{a}\left(v ; \lambda_{1}, N\right)\right) \leq\left(1+\theta_{1}\right) N$, then we stop the procedure. If $\operatorname{diam}\left(\mathcal{C}_{a}\left(v ; \lambda_{1}, N\right)\right)>\left(1+\theta_{1}\right) N$, then using Proposition 3.6 we set $\lambda_{2} \geq \lambda_{1}$ such that some part of $\mathcal{C}_{a}\left(v ; \lambda_{1}, N\right) \cap B((K+2) N)$ freezes in the time interval $\left[p_{\lambda_{1}}(N), p_{\lambda_{2}}(N)\right]$ with probability close to 1 . If indeed some part of $\mathcal{C}_{a}\left(v ; \lambda_{1}, N\right) \cap B((K+2) N)$ freezes in the time interval $\left[p_{\lambda_{1}}(N), p_{\lambda_{2}}(N)\right]$, then we iterate the procedure starting from time $p_{\lambda_{2}}(N)$. Otherwise we terminate the procedure.

Using Lemma 3.3 we set $L$ such that the event where there are at least $L$ frozen clusters intersecting $B((K+2) N)$ at time 1 has probability smaller than $\varepsilon / 2$. In each step of the procedure either the procedure stops, or the number of frozen clusters intersecting $B((K+2) N)$ increases by at least 1 . Hence the event that the procedure runs for at least $L$ steps has probability at most $\varepsilon / 2$.

Moreover, we set the parameters $\lambda_{i}, \theta_{i}$ for $i \geq 1$ above such that with probability at least $1-\varepsilon / 2$ we terminate the procedure when there are no active clusters intersecting $B(K N)$ with diameter at least $N$. Thus with probability at least $1-\varepsilon$ the procedure stops within $L$ steps, and we stop when there are no active clusters with diameter at least $N$ intersecting $B(K N)$. Hence $\lambda=\lambda_{L+1}$ satisfies the conditions of Theorem 1.3, which finishes the proof of Theorem 1.3.

Let us turn to the precise proof. By Lemma 3.3, there is $L=L(\varepsilon, K)$ and $N_{1}^{\prime}=$ $N_{1}^{\prime}(\varepsilon, K)$ such that

$$
\mathbb{P}_{N}(F C(1, K+2, N) \geq L) \leq \varepsilon / 2,
$$

where $F(t, K+2, N)$ counts the number of frozen clusters intersecting $B((K+2) N)$ at time $t \in[0,1]$.

We define the deterministic sequence $\left(\lambda_{i}, N_{i}^{\prime}, \theta_{i}, N_{i}^{\prime \prime}\right)_{i \in \mathbb{N}}$ inductively as follows. We start by setting $\lambda_{1}=0$.

Suppose that we have already defined $\lambda_{i}$ for some $i \in \mathbb{N}$. We use Proposition 3.5 to set $\theta_{i}=\theta_{i}(\varepsilon)$ and $N_{i}^{\prime \prime}=N_{i}^{\prime \prime}(\varepsilon)$ such that

$$
\mathbb{P}_{N}\left(\exists v \in B(K N) \text { s.t. } \operatorname{diam}\left(\mathcal{C}_{a}\left(v, \lambda_{i}\right)\right) \in\left[N,\left(1+\theta_{i}\right) N\right)\right)<\varepsilon 2^{-i-2}
$$

for $N \geq N_{i}^{\prime \prime}$.

Suppose that we have already defined $\theta_{i}$ for some $i \in \mathbb{N}$. Then by Proposition 3.6 we set $\lambda_{i+1}=\lambda_{i+1}(\varepsilon)$ and $N_{i+1}^{\prime}=N_{i+1}^{\prime}(\varepsilon)$ such that the probability of the intersection of the events

- $\exists v \in B(K N)$ such that $\operatorname{diam}\left(\mathcal{C}_{a}\left(v ; \lambda_{i}\right)\right) \geq\left(1+\theta_{i}\right) N$, and

- $F C\left(p_{\lambda_{i}}(N), K+2, N\right)=F C\left(p_{\lambda_{i+1}}(N), K+2, N\right)$

is less than $2^{-i-2} \varepsilon$ for $N \geq N_{i+1}^{\prime}$. Note that the event 


$$
\begin{aligned}
& \left\{F C\left(p_{\lambda_{i}}(N), K+2, N\right)=F C\left(p_{\lambda_{i+1}}(N), K+2, N\right),\right. \\
& \left.F C\left(p_{\lambda_{i}}(N), K, N\right)<F C(1, K, N)\right\}
\end{aligned}
$$

is a subset of the union of the events appearing in the definition of $\theta_{i}$ and $\lambda_{i+1}$ for $i \geq 1$. Thus the construction above gives that

$$
\mathbb{P}_{N}\left(\begin{array}{c}
F C\left(p_{\lambda_{i}}(N), K+2, N\right)=F C\left(p_{\lambda_{i+1}}(N), K+2, N,\right. \\
\left.F C\left(p_{\lambda_{i}}(N), K, N\right)<F C(1, K, N)\right)
\end{array}\right) \leq 2^{-i-1} \varepsilon
$$

for $i \geq 1$.

We set $N_{0}=\bigvee_{i=1}^{L+1}\left(N_{i}^{\prime} \vee N_{i}^{\prime \prime}\right)$. By (3.4) we have

$\mathbb{P}_{N}$ (a cluster intersecting $B(K N)$ freezes after time $\left.p_{\lambda_{L+1}}(N)\right)$

$$
\begin{aligned}
& =\mathbb{P}_{N}\left(F C\left(p_{\lambda_{L+1}}(N), K, N\right)<F C(1, K, N)\right) \\
& \leq \mathbb{P}_{N}(L<F(1, K+2, N))+\mathbb{P}_{N}\left(\begin{array}{c}
F C\left(p_{\lambda_{L+1}}(N), K+2, N\right) \leq L \\
F C\left(p_{\lambda_{L+1}}(N), K, N\right)<F C(1, K, N)
\end{array}\right) \\
& \leq \varepsilon / 2+\mathbb{P}_{N}\left(\bigcup_{i=1}^{L+1}\left\{\begin{array}{c}
F C\left(p_{\lambda_{i}}(N), K+2, N\right)=F C\left(p_{\lambda_{i+1}}(N), K+2, N\right) \\
F C\left(p_{\lambda_{i+1}}(N), K, N\right)<F C(1, K, N)
\end{array}\right\}\right) \\
& \leq \varepsilon / 2+\sum_{i=1}^{L+1} \mathbb{P}_{N}\left(\begin{array}{c}
F C\left(p_{\lambda_{i}}(N), K+2, N\right)=F C\left(p_{\lambda_{i+1}}(N), K+2, N\right) \\
F C\left(p_{\lambda_{i+1}}(N), K, N\right)<F C(1, K, N)
\end{array}\right) \\
& \leq \varepsilon / 2+\sum_{i=1}^{L+1} 2^{-i-1} \varepsilon<\varepsilon
\end{aligned}
$$

for $N \geq N_{0}$ where we applied (3.5) in the last line. This finishes the proof of Theorem 1.3 .

\subsection{Proof of Corollary 1.7}

Proof of Corollary 1.7 For $\lambda \in \mathbb{R}$ and $N \in \mathbb{N}$ let $N F(\lambda)=N F(\lambda, N)$ denote the event that no cluster intersecting $B(5 N)$ freezes after time $p_{\lambda}(N)$. By Theorem 1.3 there is $\lambda=\lambda(\varepsilon)$ and $N_{1}=N_{1}(\varepsilon)$ such that

$$
\mathbb{P}_{N}(N F(\lambda))>1-\varepsilon / 3
$$

for $N \geq N_{1}$.

First we consider the case where the origin is in an open frozen cluster at time 1 , that is $\operatorname{diam}(C(1)) \geq N$. Note that on the event $N F(\lambda)$, this frozen cluster was formed before or at $p_{\lambda}(N)$. Hence on this event there is a $p_{\lambda}(N)$-open path from the origin to distance at least $N / 2$. Hence the event $\mathcal{A}_{1,0, o}^{\lambda, \lambda, N}(1, N / 2)$ defined in Lemma 2.11 occurs. 
Let us turn to the case where $\operatorname{diam}(C(1))<N$. Recall the notation $\mathcal{C}_{a}(\lambda)$ from Definition 3.4. It is easy to check that $C(1)=\mathcal{C}_{a}(\lambda)$ on the event $\{\operatorname{diam}(C(1))<N\} \cap$ $N F(\lambda)$.

If $\operatorname{diam}\left(\mathcal{C}_{a}(\lambda)\right)<a N$, then $\partial \mathcal{C}_{a}(\lambda) \cap B(2 a N) \neq \emptyset$ for large $N$. Since $v \in$ $\partial \mathcal{C}_{a}(\lambda) \cap B(2 a N)$ is frozen, it has a neighbour which has an open frozen path to distance at least $N / 2$. On the event $N F(\lambda)$, this path is $p_{\lambda}(N)$-open. Hence the event $\mathcal{A}_{1,0, o}^{\lambda, \lambda}(2 a N, N / 2)$ occurs. This combined with the argument above, for $a \in(0,1)$ and $N>N_{2}=1 / a$ we have

$$
\{\operatorname{diam}(C(1)) \in[0, a N) \cup[N, \infty)\} \cap N F(\lambda) \subseteq \mathcal{A}_{1,0, o}^{\lambda, \lambda, N}(2 a N, N / 2) .
$$

Hence by Lemma 2.11 there is $c=c(\lambda)$ and $N_{3}=N_{3}(\lambda)$ such that

$$
\begin{aligned}
\mathbb{P}_{N}(\operatorname{diam}(C(1)) \in[0, a N) \cup[N, \infty), N F(\lambda)) & \leq \mathbb{P}\left(\mathcal{A}_{1,0, o}^{\lambda, \lambda, N}(2 a N, N / 2)\right) \\
& \leq c \mathbb{P}_{1 / 2}\left(\mathcal{A}_{1, o}(2 a N, N / 2)\right)
\end{aligned}
$$

for $N \geq N_{3}$. Theorem 2.6 gives that there is $a=a(\varepsilon)$ and $N_{4}=N_{4}(\varepsilon)$ such that

$$
\mathbb{P}_{N}(\operatorname{diam}(C(1)) \in[0, a N) \cup[N, \infty), N F(\lambda)) \leq c \mathbb{P}_{1 / 2}\left(\mathcal{A}_{1, o}(2 a N, N / 2)\right)<\varepsilon / 3
$$

for $N \geq N_{4}$.

Finally, Proposition 3.5 gives $b=b(\varepsilon)$ and $N_{5}=N_{5}(\varepsilon)$ such that

$$
\begin{aligned}
\mathbb{P}_{N}\left(\operatorname{diam}\left(\mathcal{C}_{a}(\lambda)\right) \in[b N, N), N F(\lambda)\right) & \leq \mathbb{P}_{N}\left(\operatorname{diam}\left(\mathcal{C}_{a}(\lambda)\right) \in[b N, N)\right) \\
& \leq \varepsilon / 3
\end{aligned}
$$

for $N \geq N_{5}$.

Since $C(1)=\mathcal{C}_{a}(\lambda)$ on the event $\{\operatorname{diam}(C(1))<N\} \cap N F(\lambda)$, a combination of (3.6), (3.7) and (3.8) finishes the proof of Corollary 1.7.

\section{Proof of Proposition 3.6}

\subsection{Notation}

Let us introduce some more notation. For $u=\left(u_{1}, u_{2}\right), v=\left(v_{1}, v_{2}\right) \in V$, we say that $u$ is left (right, resp.) of $v$ if $u_{1} \leq v_{1}\left(u_{1} \geq v_{1}\right.$, resp.). Similarly we say that $u$ is below (above, resp.) $v$ if $u_{2} \leq v_{2}\left(u_{2} \geq v_{2}\right)$. For a finite set of vertices $W \subseteq V$ we say that $v=\left(v_{1}, v_{2}\right) \in W$ is a leftmost (rightmost, resp.) vertex of $W$ if for all $w=\left(w_{1}, w_{2}\right) \in W, v_{1} \leq w_{1}\left(v_{1} \geq w_{1}\right.$, resp. $)$. We define the lowest and highest vertices of $W$ in an analogous way.

Recall that for $v, w \in V, v \sim w$ denotes that $v$ and $w$ are neighbours in $\mathbb{T}$. We extend this notation for subsets of $V:$ For $S, U \subset V, S \sim U$ denotes that $\exists s \in S, \exists u \in U$ such that $s \sim u$. Moreover, $S \nsim U$ denotes that $S \sim U$ does not hold. 
Definition 4.1 Let $n \in \mathbb{N}$. We say that a sequence of vertices $v^{1}, v^{2}, \ldots, v^{n}$, denoted by $\rho$, is a path if

- $v^{i} \sim v^{i+1}$ for $i=1,2, \ldots,(n-1)$, and

- $v^{i} \neq v^{j}$ when $i \neq j \quad$ for $i, j=1,2, \ldots, n$.

We say that $\rho$ is non self touching, two of its vertices are adjacent, then they are consecutive. That is, if $u, w \in \rho$ with $u \sim w$ then there is some $i \in \mathbb{N}$ with $1 \leq i \leq$ $n-1$ such that either $(u, v)=\left(v^{i}, v^{i+1}\right)$ or $(u, v)=\left(v^{i+1}, v^{i}\right)$. We consider our paths to be ordered: $v^{1}$ is the starting point and $v^{n}$ is the ending point of $\rho$. For $u, w \in \rho$ we say that $u$ is after $w$ in $\rho$, and denote it by $w \prec_{\rho} u$ if $u=v^{i}$ and $w=v^{j}$ for some $i, j \in \mathbb{N}$ with $1 \leq j<i \leq n$. For $u, w \in \rho, u \preceq_{\rho} w$ denotes that either $u=w$ or $u \prec \rho w$. When it is clear from the context which path we are considering, we omit the subscript $\rho$. For $u, w, z \in \rho$ we say that $w$ is in between $u$ and $z$ if $u \preceq w \preceq z$ or $u \succeq w \succeq z$. For $u, z \in \rho$ with $u \preceq \rho z$ let $\rho_{u, z}$ denote the subpath of $\rho$ consisting of the vertices between $u$ and $z$.

We say that two paths $\rho_{1}, \rho_{2}$ are non-touching, if $\rho_{1} \nsim \rho_{2}$.

Definition 4.2 Let $n \in \mathbb{N}$ and sequence of vertices $v^{1}, v^{2}, \ldots, v^{n}$, satisfying

- $v^{i} \sim v^{i+1} \bmod n$ for $i=1,2, \ldots, n$, and

- $v^{i} \neq v^{j}$ when $i \neq j$ for $i, j=1,2, \ldots, n$.

A loop $v$ is the equivalence class of the sequence $\left(v^{1}, v^{2}, \ldots, v^{n}\right)$ under cyclic permutations, i.e $v$ is the set of sequences $\left(v^{j}, v^{j+1} \bmod n, \ldots, v^{j+n-1} \bmod n\right)$ for $j=1,2, \ldots, n . v$ is non-self touching if for all $\left(w^{1}, w^{2}, \ldots, w^{n}\right) \in v$, the path $\left(w^{1}, w^{2}, \ldots, w^{n-1}\right)$ is non-self touching.

With a slight abuse of notation, we say that a loop $v$ contains a vertex $v$ and denote it by $v \in v$ if $v=v^{i}$ for some $i \in\{1,2, \ldots, n\}$. Let $v, w \in v$ with $v \neq w$ and let $\rho$ denote the unique path which starts at $v$ and represents $v$. With the notation of Definition 4.1, let $v_{v, w}:=\rho_{v, w}$ denote the arc of $v$ starting at $v$ and ending at $w$.

\subsection{Thick paths}

Definition 4.3 Let $M \in \mathbb{N}$ be fixed. The $M$-grid is the set of parallelograms $B((2 M+1) z ; M)$ for $z \in V$. Let $\pi$ be a sequence consisting of some parallelograms of the $M$-grid. We say that $\pi$ is an $M$-gridpath, if for any two consecutive parallelograms $B, B^{\prime}$ of $\pi$ share a side, i.e $\left|\partial B \cap B^{\prime}\right| \geq 2$.

Definition 4.4 Let $C$ be a subgraph of $\mathbb{T}, D \subset V$ and $a, b \in \mathbb{N}$. We say that $C$ is $(a, b)$-nice in $D$, if it satisfies the conditions

1. $C$ is a connected induced subgraph of $\mathbb{T}$,

2. $\partial C$ is a disjoint union of non-touching loops, each with diameter bigger than $2 b$.

3. Let $u, v \in \partial C \cap D$ with $d(u, v) \leq a$. Then $u, v$ are contained in the same loop $\gamma$ of $\partial C$, and $\operatorname{diam}\left(\gamma_{u, v}\right) \wedge \operatorname{diam}\left(\gamma_{v, u}\right) \leq b$.

In the case where $D=V$, we say that $C$ is $(a, b)$-nice. 
Let $C$ be $(a, b)$-nice for some $a, b \in \mathbb{N}$. Condition 3 of Definition 4.4, roughly speaking, says that if there is a corridor in $C$ with width less than $a$, then it connects two parts of $C$ such that one part has diameter at most $b$. This suggests that when $b$ is small compared to $\operatorname{diam}(C)$, then we can move a parallelogram with side length $O(a)$ in $C$ between two distant points of $C$. This intuitive argument leads us to the following lemma.

Lemma 4.5 Let $a, b \in \mathbb{N}$ with $a \geq 2000$. Let $C$ be an $(a, b)$-nice subgraph of $\mathbb{T}$. Then there is a $\lfloor a / 200-10\rfloor$-gridpath contained in $C$ with diameter at least $\operatorname{diam}(C)-2 b-2 a-12$.

We use the following 'local' version of Lemma 4.5:

Lemma 4.6 Let $a, b, c \in \mathbb{N}$ with $a \geq 2000$. Let $C$ be subgraph of $\mathbb{T}$ which is $(a, b)$-nice in $B(c)$. Let $C^{\prime}$ be a connected component of $C \cap B(c)$. Then there is a $\lfloor a / 200-10\rfloor$-gridpath contained in $C^{\prime}$ with diameter at least diam $\left(C^{\prime}\right)-2 b-2 a-12$.

Proof of Lemmas 4.5 and 4.6 The proof of Lemmas 4.5 and 4.6 have a geometric/topologic nature, hence they are moved to Sect. 6.2 of the Appendix.

We recall and prove Proposition 3.6 in the following.

Proposition 3.6 Let $\theta \in(0,1), \varepsilon, K>0$ and $\lambda_{1} \in \mathbb{R}$. Recall the notation $F C(t, K+2, N)$ from Lemma 3.3. There exist $\lambda_{2}=\lambda_{2}\left(\lambda_{1}, \theta, \varepsilon\right)$ and $N_{0}=$ $N_{0}\left(\lambda_{1}, \theta, \varepsilon\right)$ such that the probability of the intersection of the events

- $\exists v \in B(K N)$ such that $\operatorname{diam}\left(\mathcal{C}_{a}\left(v ; \lambda_{1}, N\right)\right) \geq(1+\theta) N$, and

- none of the clusters intersecting $B((K+2) N)$ freeze in the time interval $\left(p_{\lambda_{1}}(N), p_{\lambda_{2}}(N)\right]$, i.e.

$$
F C\left(p_{\lambda_{1}}(N), K+2, N\right)=F C\left(p_{\lambda_{2}}(N), K+2, N\right)
$$

is less than $\varepsilon$ for $N \geq N_{0}$.

Proof of Proposition 3.6 By Lemma 2.27 we choose $\lambda_{0}=\lambda_{0}(\varepsilon, K) \leq \lambda_{1}$ and $N_{1}=$ $N_{1}(\varepsilon, K)$ such that

$$
\mathbb{P}\left(\mathcal{N}_{c}\left(\lambda_{0}, 1 / 6, K+6, N\right)\right)>1-\varepsilon / 3 \text {. }
$$

By Corollary 2.13 we choose $\eta<\theta / 10$ and $N_{2}=N_{2}\left(\eta, \theta, \lambda_{0}, \lambda_{1}, K\right)$ such that

$$
\mathbb{P}\left(\mathcal{N} \mathcal{A}\left(2 \eta, \theta / 10, \lambda_{0}, \lambda_{1}, K+4, N\right)\right)>1-\varepsilon / 3
$$

for all $N \geq N_{2}$. Let

$$
E:=\mathcal{N}_{c}\left(\lambda_{0}, 1 / 6, K+6, N\right) \cap \mathcal{N} \mathcal{A}\left(2 \eta, \theta / 10, \lambda_{0}, \lambda_{1}, K+4, N\right) .
$$

Claim 4.7 Let $u \in B(K N)$ with $\operatorname{diam}\left(\mathcal{C}_{a}\left(u ; \lambda_{1}, N\right)\right) \geq(1+\theta) N$. Then $\mathcal{C}_{a}\left(u ; \lambda_{1}, N\right)$ is $\left(\eta N, \frac{\theta}{10} N\right)$-nice in $B(u ; 2 N)$ on the event $E$. 
Proof of Claim 4.7 Let us check the conditions of Definition 4.4. Condition 1 is satisfied by the definition of $\mathcal{C}_{a}\left(u ; \lambda_{1}, N\right)$.

All the holes of $\mathcal{C}_{a}\left(u ; \lambda_{1}, N\right)$ contain a frozen cluster, which have diameter at least $N$. This combined with $2 \frac{\theta}{10} N<N$, shows that Condition 2 of Definition 4.4 holds.

Let $x, y \in \partial \mathcal{C}_{a}\left(u ; \lambda_{1}, K\right) \cap B(u ; 2 N)$ with $d(x, y) \leq \eta N$. We have two cases.

Case 1. $x, y$ lie in different loops of $\partial \mathcal{C}_{a}\left(u ; \lambda_{1}, N\right)$. For $i=x, y$, let $\gamma_{i}$ denote the loop containing $i$. Furthermore, let $\tilde{\gamma}_{i}$ denote the connected component of $i$ in $\gamma_{i} \cap B(i ; 2 N)$. We have $\operatorname{diam}\left(\tilde{\gamma}_{i}\right) \geq N$. Moreover, $\tilde{\gamma}_{i} \subset B(i ; 2 N) \subset$ $B((K+4) N)$. Observation 3.2 gives that on the event $\mathcal{N}_{c}\left(\lambda_{0}, 1 / 6, K+6, N\right), \tilde{\gamma}_{i}$ is $p_{\lambda_{0}}(N)$-closed. Hence each of $\tilde{\gamma}_{x}$ and $\tilde{\gamma}_{y}$ gives two closed $p_{\lambda_{0}}(N)$-closed arms in $A(x ; 2 \eta N, N / 2)$. Moreover, the frozen clusters neighbouring $x$ and $y$ provide two disjoint $p_{\lambda_{1}}(N)$-open arms. Hence there are 6 disjoint arms in $A(x ; 2 \eta N, N / 2)$, thus $\mathcal{N} \mathcal{A}^{c}\left(2 \eta, \theta / 10, \lambda_{0}, \lambda_{1}, K+4, N\right)$ occurs.

Case 2. $x, y$ lie on the same loop of $\partial \mathcal{C}_{a}\left(u ; \lambda_{1}, N\right)$. This case can be treated similarly to Case 1, with the difference that if $x, y$ violate Condition 3 of Definition 4.4 then we get 6 arms in $A\left(x ; 2 \eta N, \frac{\theta}{10} N\right)$. Hence $\mathcal{N} \mathcal{A}^{c}\left(2 \eta, \theta / 10, \lambda_{0}, \lambda_{1}, K+4, N\right)$ occurs.

Hence in both cases $E^{c}$ occurs. Thus on the event $E$ all the conditions of Definition 4.4 are satisfied for $\mathcal{C}_{a}\left(u ; \lambda_{1}, N\right)$, which finishes the proof of Claim 4.7.

Let us turn back to the proof of Proposition 3.6. Let $u \in B(K N)$ with $\operatorname{diam}\left(\mathcal{C}_{a}\left(u ; \lambda_{1}, N\right)\right) \geq(1+\theta) N$. Let $\tilde{\mathcal{C}}_{a}\left(u, \lambda_{1}, N\right)$ denote the connected component of $u$ in $\mathcal{C}_{a}\left(u, \lambda_{1}, N\right) \cap B(u ; 2 N)$. Since $\operatorname{diam}\left(\mathcal{C}_{a}\left(u ; \lambda_{1}, N\right)\right) \geq(1+\theta) N$ and $\theta<1$, we have $\operatorname{diam}\left(\tilde{\mathcal{C}}_{a}\left(u ; \lambda_{1}, N\right)\right) \geq(1+\theta) N$. By Lemma 4.6 we set $\eta=\eta(\theta) \in(0, \theta / 100)$ and $N_{3}=N_{3}(\theta)$ such that on the event $E$, for all $u \in B(K N)$ with $\operatorname{diam}\left(\mathcal{C}_{a}\left(u ; \lambda_{1}, N\right)\right) \geq(1+\theta) N$ there is a $\lfloor\eta N\rfloor$-gridpath $\rho_{u} \subset \tilde{\mathcal{C}}_{a}\left(u ; \lambda_{1}, N\right)$ with $\operatorname{diam}\left(\rho_{u}\right) \geq(1+\theta / 2) N$ for $N \geq N_{3}$.

Lemma 2.27 gives that there is $\lambda_{2}=\lambda_{2}(\varepsilon, \eta, K)$ and $N_{4}=N_{4}(\varepsilon, \eta, K)$ such that

$$
\mathbb{P}\left(\mathcal{N}_{o}\left(\lambda_{2}, \eta / 2, K+4, N\right)\right)>1-\varepsilon / 3
$$

for $N \geq N_{4}(\varepsilon, \eta, K)$. We set $N_{0}:=\bigvee_{i=1}^{4} N_{i}$. Let

$$
\begin{aligned}
G & :=E \cap \mathcal{N}_{o}\left(\lambda_{2}, \eta / 2, K+4, N\right), \\
M & :=\left\{\exists v \in B(K N) \text { s.t. } \operatorname{diam}\left(\mathcal{C}_{a}\left(v ; \lambda_{1}, N\right)\right) \geq(1+\theta) N\right\} \cap G .
\end{aligned}
$$

Combination of (4.1), (4.2) and (4.3) gives that

$$
\mathbb{P}\left(G^{c}\right)<\varepsilon
$$

for $N \geq N_{0}$.

Recall that for $N \geq N_{0}$, on the event $E$ for $u \in B(K N)$, with $\operatorname{diam}\left(\mathcal{C}_{a}\left(u ; \lambda_{1}, N\right)\right) \geq$ $(1+\theta) N$ there is a $\lfloor\eta N\rfloor$-gridpath $\rho_{u} \subset \tilde{\mathcal{C}_{a}}\left(u ; \lambda_{1}, N\right)$ with $\operatorname{diam}\left(\rho_{u}\right) \geq(1+\theta / 2) N$. On the event $\mathcal{N}_{o}\left(\lambda_{2}, \eta / 2, K+4, N\right)$, this gridpath $\rho_{u} \subseteq B((K+2) N)$ contains a $p_{\lambda_{2}}(N)$-open component with diameter at least $N$. Hence on the event $M$, at least one cluster intersecting $B((K+2) N)$ freezes in the time interval $\left(p_{\lambda_{1}}(N), p_{\lambda_{2}}(N)\right]$. 
That is

$$
M \subseteq\left\{F C\left(p_{\lambda_{1}}(N), K+2, N\right)<F C\left(p_{\lambda_{2}}(N), K+2, N\right)\right\}
$$

Thus

$$
\begin{aligned}
\{\exists v & \left.\in B(K N) \text { s.t. } \operatorname{diam}\left(\mathcal{C}_{a}\left(v ; \lambda_{1}, N\right)\right) \geq(1+\theta) N\right\} \cap\left\{F C\left(p_{\lambda_{1}}(N), K+2, N\right)\right. \\
& \left.=F C\left(p_{\lambda_{2}}(N), K+2, N\right)\right\} \subset G^{c},
\end{aligned}
$$

which together with (4.4) finishes the proof of Proposition 3.6.

\section{Proof of Proposition 3.5}

5.1 Lowest point of the lowest crossing in parallelograms

Recall the notation of Sect. 4.1.

Definition 5.1 Let $R$ be a connected subgraph of $\mathbb{T}$ and let $r \subset \partial R$. We define $\mathcal{L}(R, r)$ as the (random) set of lowest vertices $v \in R$ such that $v$ is closed, and there are two non-touching closed paths in $R$ starting at a vertex neighbouring to $v$ and ending at $r$.

Consider the site percolation model on the triangular lattice with parameter $p \in[0,1]$. We investigate the distribution of $\mathcal{L}(R, r)$ in the case where $p=p_{\lambda}(N)$, $R=B(b N)$ and $r=\operatorname{top}(B(b N)):=[-b N, b N] \otimes\{\lfloor b N\rfloor+1\}$ for $\lambda \in \mathbb{R}$ and $b>0$.

Definition 5.2 For a parallelogram $B$, let $\operatorname{HCr}(B)$ denote set of paths in $B$ which connect the left and the right sides of $B$. For $\rho \in \operatorname{HCr}(B)$, let $B e(\rho)=B e(\rho, B)$ denote the set of vertices in $B$ which are 'under' $\rho$. It is the set of vertices $v \in B \backslash \rho$ which are connected to the bottom side of $B$. Furthermore, we define $A b(\rho)=$ $A b(\rho, B):=B \backslash(\rho \cup B e(\rho, B))$.

Lemma 5.3 Let $a, b \in(0,1)$ with $5 a<b$. For $k, l, N \in \mathbb{N}$ with $l<k$ we define the parallelogram

$$
B_{l, k}:=[-a N, a N] \otimes\left(\left(2 \frac{l}{k}-1\right) a N,\left(2 \frac{l+1}{k}-1\right) a N\right]
$$

and the event

$$
L_{l, k}=:\left\{\mathcal{L}(B(b N), \text { top }(b N)) \cap B_{l, k} \neq \emptyset\right\} .
$$

That is, $L_{l, k}$ is the event that at least one of the lowest vertices of $B(b N)$ with two nontouching closed paths $B(b N)$ to the top side of $B(b N)$ is in the parallelogram $B_{l, k}$.

Let $\lambda_{1}, \lambda_{2} \in \mathbb{R}$. Then there exist $C=C\left(a, b, \lambda_{1}, \lambda_{2}\right)$ and $N_{0}=N_{0}\left(a, b, \lambda_{1}, \lambda_{2}, k\right)$ such that for all $\lambda \in\left[\lambda_{1}, \lambda_{2}\right]$ and $k, l \in \mathbb{N}$ with $l \leq k-1$ we have

$$
\mathbb{P}_{p_{\lambda}(N)}\left(L_{l, k}\right) \leq C k^{-1}
$$

for $N \geq N_{0}$. In particular, the upper bound in (5.3) is uniform in $l$. 


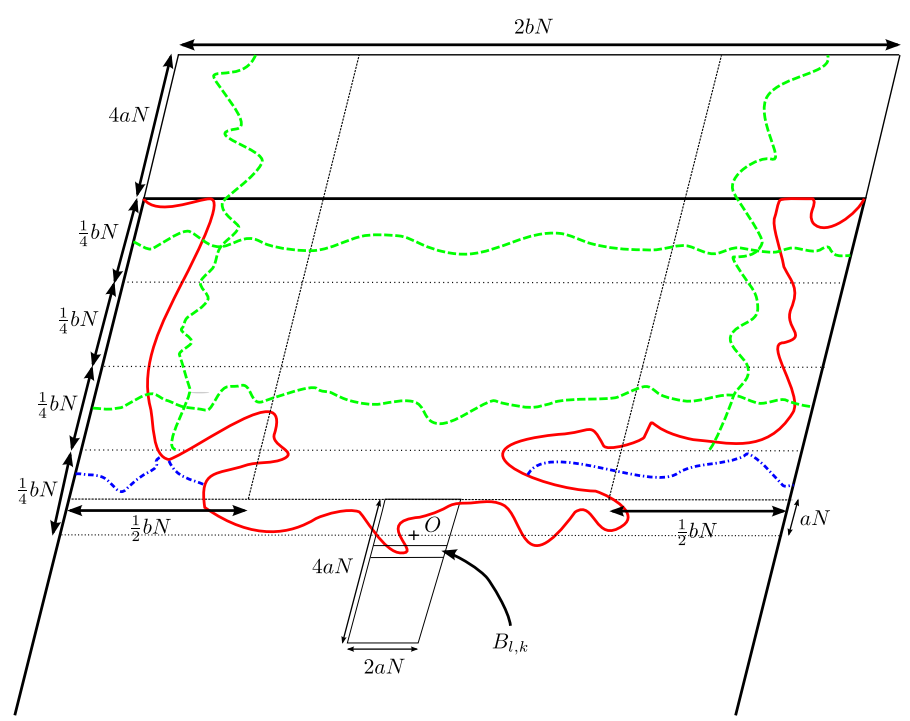

Fig. 1 The continuous line represents $\gamma$. The dashed paths are the closed crossings of $\mathcal{D}$, which allow us to prolong $\gamma$. The dashed-dotted paths are the open parts of $\xi(\gamma)$. They, together with $\gamma$, prevent the occurrence of closed vertices below the lowest point of $\gamma$ with two closed arms to the top side of $B(b N)$ after the shift

Proof of Lemma 5.3 For $k \leq 5$ the statement is trivial, hence we assume that $k \geq 5$ in the following. We extend the notation in (5.1) and (5.2) for $l \in\{-k,-k+1, \ldots,-1\}$.

First we show that there exist $c=c\left(a, b, \lambda_{1}, \lambda_{2}\right)>0$ and $N_{0}=N_{0}\left(a, b, \lambda_{1}, \lambda_{2}\right)$ such that for all $l, m \in[-k, k-1] \cap \mathbb{Z}$ with $m+1 \leq l$ we have

$$
c \mathbb{P}_{p_{\lambda}(N)}\left(L_{l, k}\right) \leq \mathbb{P}_{p_{\lambda}(N)}\left(L_{m, k} \cup L_{m+1, k}\right)
$$

for $N \geq N_{0}$. Let $S=S(l, m, k): V \rightarrow V$ denote a shift which moves the parallelogram $B_{l, k}$ to a subset of $B_{m, k} \cup B_{m+1, k}$. The shift $S$ naturally induces a map on the configurations $\omega \in\{o, c\}^{V}$ by $S(\omega)(v)=\omega\left(S^{-1}(v)\right)$. Roughly speaking, we prove (5.4) by showing that positive proportion of the configurations $\omega \in L_{l, k}$ satisfy $S(\omega) \in L_{m, k} \cup L_{m+1, k}$. We achieve this by showing that, conditioning on $L_{l, k}$, all the crossing events of Fig. 1 occur with probability bounded away from 0 . Let us turn to the precise proof.

Let $k, l$ be given. Let $s_{L}\left(s_{R}\right.$, resp.) denote the left (right, resp.) endpoint of top $(b N)$. We say that a path $\rho \subseteq B(b N) \cup \operatorname{top}(b N)$ is good, if it

- starts at $s_{L}$ and ends at $s_{R}$,

- it is non-self touching,

- and one of its lowest points is in $B_{l, k}$.

Let $\rho$ be some given good path. Recall Definition 5.2 and let $B e(\rho)=B e(\rho,(B(b N)))$. Let $H_{\rho}$ denote the event that there are two open paths in $B e(\rho) \cap[-b N, b N] \otimes$ $\left[a N, \frac{b-2 a}{2} N\right]$ from the left and right sides of the parallelogram $[-b N, b N] \otimes$ 
$\left[a N, \frac{b-2 a}{2} N\right]$ to $\rho$. Let $\gamma$ denote the lowest non-self touching path in $B(b N) \cup$ top $(b N)$ which starts at $s_{L}$ and ends at $s_{R}$, and of which all the vertices outside of top $(b N)$ are closed. On the event $L_{l, k} \gamma$ is good.

Let $\rho$ be a fixed good path. Let $O_{\rho}$ denote the event that there is path $v$ such that

- $v \subseteq B_{0}:=[-b N, b N] \otimes\left[-b N, \frac{b}{4} N\right]$,

- $v$ connects the left and the right sides of the parallelogram $B_{1}:=[-b N, b N] \otimes$ $\left[a N, \frac{b}{4} N\right]$

- $v$ is a concatenation of some open paths which lie in $B e(\rho) \cap B_{1}$, and of some subpaths of $\rho$.

Clearly, $O_{\rho}$ is an increasing event. On $O_{\rho}$, let $\xi(\rho)$ denote the lowest path which satisfies the conditions in the definition of $O_{\rho}$. Recall the definition of decreasing events from Definition 2.1, and the definition of $\gamma$ from Case 1. Let us condition on the event that all the vertices of $\rho \backslash$ top $(b N)$ are closed. Then the event $\{\gamma=\rho\}$ is increasing on the configuration in $B(b N) \backslash \rho$, and it only depends on the configuration in $B e(\rho)$. Hence a combination of the FKG inequality and Corollary 2.20 gives that

$$
\begin{aligned}
& \mathbb{P}_{p_{\lambda}(N)}\left(L_{l, k} \cap O_{\gamma}\right) \\
&=\sum_{\rho \operatorname{good}} \mathbb{P}_{p_{\lambda}(N)}\left(O_{\rho} \cap\{\gamma=\rho\} \mid \rho \backslash \text { top }(b N) \text { is closed }\right) \\
& \quad \times \mathbb{P}_{p_{\lambda}(N)}(\rho \backslash \text { top }(b N) \text { is closed }) \\
& \geq \sum_{\rho \operatorname{good}} \mathbb{P}_{p_{\lambda}(N)}(\{\gamma=\rho\} \mid \rho \backslash \text { top }(b N) \text { is closed }) \\
& \quad \times \mathbb{P}_{p_{\lambda}(N)}\left(O_{\rho} \mid \rho \backslash \text { top }(b N) \text { is closed }\right) \mathbb{P}_{p_{\lambda}(N)}(\rho \backslash \text { top }(b N) \text { is closed }) \\
& \geq \sum_{\rho \operatorname{good}} \mathbb{P}_{p_{\lambda}(N)}(\{\gamma=\rho\} \mid \rho \backslash \text { top }(b N) \text { is closed }) \\
& \times \mathbb{P}_{p_{\lambda}(N)}\left(\mathcal{H}_{o}\left(B_{1}\right)\right) \mathbb{P}_{p_{\lambda}(N)}(\rho \backslash \text { top }(b N) \text { is closed }) \\
& \geq c_{1}\left(\lambda_{1}, \lambda_{2}, a, b\right) \mathbb{P}_{p_{\lambda}(N)}\left(L_{l, k}\right)
\end{aligned}
$$

for $c_{1}=c_{1}\left(a, b, \lambda_{1}, \lambda_{2}\right)>0$ and for $N \geq N_{1}=N_{1}\left(a, b, \lambda_{1}, \lambda_{2}\right)$.

For $W \subseteq V$ and $\omega \in\{o, c\}^{V}, \omega_{W} \in\{o, c\}^{W}$ denotes the restriction of $\omega$ to the configuration in $W$. That is $\omega_{W}(v)=\omega(v)$ for $v \in W$. Recall Definition 5.2. Let $\zeta \in H C r\left(B_{0}\right)$ be arbitrary. It is easy to check that the event $L_{l, k} \cap O_{\gamma} \cap\{\xi(\gamma)=\zeta\}$ is decreasing in the configuration in $A b(\zeta)$. Let us take the parallelograms

$$
\begin{aligned}
B_{2} & :=[-b N, b N] \otimes\left[\frac{b}{4} N, \frac{b}{2} N\right], \\
B_{3} & :=[-b N, b N] \otimes\left[\frac{3}{4} b N, b N\right], \\
B_{4} & :=\left[-b N,-\frac{1}{2} b N\right] \otimes\left[\frac{1}{4} b N,(b+4 a) N\right]
\end{aligned}
$$




$$
B_{5}:=\left[\frac{1}{2} b N, b N\right] \otimes\left[\frac{1}{4} b N,(b+4 a) N\right] .
$$

Let

$$
\mathcal{D}:=\mathcal{H}_{c}\left(B_{2}\right) \cap \mathcal{H}_{c}\left(B_{3}\right) \cap \mathcal{V}_{c}\left(B_{4}\right) \cap \mathcal{V}_{c}\left(B_{5}\right)
$$

Clearly, $\mathcal{D}$ is a decreasing event. Hence a combination of the FKG inequality and Corollary 2.20 gives that for $c_{2}=c_{2}\left(a, b, \lambda_{1}, \lambda_{2}\right)>0$ and $N \geq N_{2}=N_{2}\left(a, b, \lambda_{1}, \lambda_{2}\right)$ we have

$$
\begin{aligned}
& \mathbb{P}_{p_{\lambda}(N)}\left(L_{k, l} \cap O_{\gamma} \cap \mathcal{D}\right) \\
&=\sum_{\zeta} \sum_{\sigma} \mathbb{P}_{p_{\lambda}(N)}\left(L_{k, l} \cap O_{\gamma} \cap\{\xi(\gamma)=\zeta\} \cap \mathcal{D} \mid \omega_{\zeta \cup B e(\zeta)}=\sigma\right) \\
& \quad \times \mathbb{P}_{p_{\lambda}(N)}\left(\omega_{\zeta \cup B e(\zeta)}=\sigma\right) \\
& \geq \sum_{\zeta} \sum_{\sigma} \mathbb{P}_{p_{\lambda}(N)}\left(L_{k, l} \cap O_{\gamma} \cap\{\xi(\gamma)=\zeta\} \mid \omega_{\zeta \cup B e(\zeta)}=\sigma\right) \\
& \quad \times \mathbb{P}_{p_{\lambda}(N)}\left(\mathcal{D} \mid \omega_{\zeta \cup B e(\zeta)}=\sigma\right) \mathbb{P}_{p_{\lambda}(N)}\left(\omega_{\zeta \cup B e(\zeta)}=\sigma\right) \\
&=\sum_{\zeta} \sum_{\sigma} \mathbb{P}_{p_{\lambda}(N)}\left(L_{k, l} \cap O_{\gamma} \cap\{\xi(\gamma)=\zeta\} \mid \omega_{\zeta \cup B e(\zeta)}=\sigma\right) \\
& \quad \times \mathbb{P}_{p_{\lambda}(N)}\left(\mathcal{D}_{)} \mathbb{P}_{p_{\lambda}(N)}\left(\omega_{\zeta \cup B e(\zeta)}=\sigma\right)\right. \\
& \geq c_{2}\left(a, b, \lambda_{1}, \lambda_{2}\right) \sum_{\zeta} \sum_{\sigma} \\
& \times \mathbb{P}_{p_{\lambda}(N)}\left(L_{k, l} \cap O_{\gamma} \cap\{\xi(\gamma)=\zeta\} \mid \omega_{\zeta \cup B e(\zeta)}=\sigma\right) \mathbb{P}_{p_{\lambda}(N)}\left(\omega_{\zeta \cup B e(\zeta)}=\sigma\right) \\
&= c_{2}\left(a, b, \lambda_{1}, \lambda_{2}\right) \mathbb{P}_{p_{\lambda}(N)}\left(L_{k, l} \cap O_{\gamma}\right)
\end{aligned}
$$

where the summation in $\zeta$ is over $\operatorname{HCr}\left(B_{0}\right)$ and the summation in $\sigma$ is over $\{o, c\}^{\zeta \cup B e(\zeta)}$. In the third line we used that $\mathcal{D}$ does not depend on the configuration in $\zeta \cup B e(\zeta)$.

There is $N_{3}=N_{3}(k)$ such that for $N \geq N_{3}$ and for all $l, m \in[0, k-1] \cap \mathbb{Z}$ with $l>m$ there is a shift $S=S(l, m, k)$ which moves the parallelogram $B_{l, k}$ to a subset of $B_{m, k} \cup B_{m+1, k}$. Let us take a configuration $\omega \in\{o, c\}^{V}$ which satisfies $L_{k, l} \cap O_{\gamma} \cap \mathcal{D}$. Then the shifted configuration $S(\omega)$ satisfies $L_{m, k} \cup L_{m+1, k}$. See Fig. 1 for more details. Hence for $N \geq N_{1} \vee N_{2} \vee N_{3}$ we have

$$
\begin{aligned}
\mathbb{P}_{p_{\lambda}(N)}\left(L_{m, k} \cup L_{m+1, k}\right) & \geq \mathbb{P}_{p_{\lambda}(N)}\left(L_{k, l} \cap O_{\gamma} \cap \mathcal{D}\right) \\
& \geq c_{1} c_{2} \mathbb{P}_{p_{\lambda}(N)}\left(L_{l, k}\right)
\end{aligned}
$$

by a combination of (5.5) and (5.6). This finishes the proof of (5.4). Now we conclude the proof of Lemma 5.3. By summing over $m \in\{-k,-k+1, \ldots,-2\}$ in (5.7) we get that 


$$
\begin{aligned}
\mathbb{P}_{p_{\lambda}(N)}\left(L_{k, l}\right) & \leq(k-1)^{-1} c_{1} c_{2} \sum_{m=-k}^{-2} \mathbb{P}_{p_{\lambda}(N)}\left(L_{m, k} \cup L_{m+1, k}\right) \\
& \leq 2 c_{1} c_{2} k^{-1} \sum_{m=-k}^{-1} \mathbb{P}_{p_{\lambda}(N)}\left(L_{m, k}\right) \\
& \leq C k^{-1}
\end{aligned}
$$

for some $C=C\left(a, b, \lambda_{1}, \lambda_{2}\right)$. In the last line we used that $L_{m, k} \cap L_{m^{\prime}, k}=\emptyset$ for $m \neq m^{\prime}$. This finishes the proof of Lemma 5.3.

Remark 5.4 Let $a, b, \lambda, \lambda_{1}, \lambda_{2}$ be as in Lemma 5.3. Standard RSW techniques give that there is $c^{\prime}=c^{\prime}\left(a, b, \lambda_{1}, \lambda_{2}\right)>0$ and $N_{0}=N_{0}\left(a, b, \lambda_{1}, \lambda_{2}\right)$ such that

$$
\mathbb{P}_{p_{\lambda}(N)}(\mathcal{L}(B(b N), t(b N)) \cap B(a N) \neq \emptyset) \geq c^{\prime}
$$

for $N \geq N_{0}$. This, combined with arguments similar to the proof of Lemma 5.3, gives that there is $C^{\prime}=C^{\prime}\left(a, b, \lambda_{1}, \lambda_{2}\right)>0$ and $N_{1}=N_{1}\left(a, b, \lambda_{1}, \lambda_{2}, k\right)$ such that

$$
\mathbb{P}_{p_{\lambda}(N)}\left(L_{l, k}\right) \geq C^{\prime} k^{-1}
$$

for $N \geq N_{1}$ uniformly for $l \leq k$.

\subsection{Lowest point of the lowest crossing in regular regions}

Recall Definition 5.1. Let $B \subset B^{\prime}$ be parallelograms, and let $R$ be a subgraph of $\mathbb{T}$ with $B \subset R \subset B^{\prime}$. Furthermore let $r \subset \partial R$. Our next aim is to compare the event $\mathcal{L}(R, r) \cap B \neq \emptyset$ to $\mathcal{L}\left(B^{\prime}\right.$, top $\left.\left(B^{\prime}\right)\right) \cap B \neq \varnothing$ in the case where the pair $(R, r)$ is 'regular'. We make this precise in the following.

We say that a subgraph $H \subseteq \mathbb{T}$ is simply connected, if it is connected and for all loops $\sigma \subseteq H$, all of the finite components of $\mathbb{T} \backslash \sigma$ are contained in $H$.

Definition 5.5 Let $a, b \in \mathbb{N}$ such that $5 a<b$. A pair $(R, r)$ is $(a, b)$-regular, if

1. $R$ is a connected induced subgraph of $\mathbb{T}$,

2. $B(a) \subseteq R \subseteq B(b)$,

3. $r \subset \partial R$, such that $\emptyset \neq r \varsubsetneqq \partial R$. Furthermore, $r$ and $\partial R \backslash r$ are self-avoiding paths such that $R$ is on the right hand side, as we walk along them.

4. $r \subseteq[-b, b] \otimes[5 a, b]$.

Lemma 5.6 Let $a, b \in(0,1)$ with $5 a<b$ and $\lambda \in \mathbb{R}$. Let $(R, r)$ be $(a N, b N)$-regular. For $k, l, N \in \mathbb{N}$ with $l<k$ we define the events

$$
\begin{aligned}
L_{l, k}(B(2 b N), \text { top }(B(2 b N))): & =\left\{\mathcal{L}(B(2 b N), \text { top }(2 b N)) \cap B_{l, k} \neq \emptyset\right\}, \\
L_{l, k}(R, r): & =\left\{\mathcal{L}(R, r) \cap B_{l, k} \neq \emptyset\right\},
\end{aligned}
$$




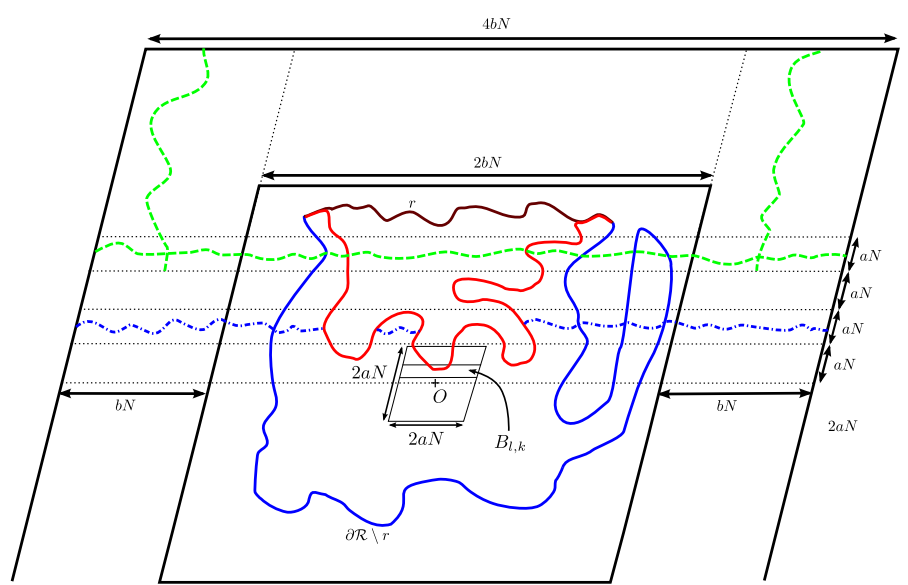

Fig. 2 The dashed paths are the closed crossings of the event $\mathcal{D}$ which allow us to prolong $\gamma$. The dasheddotted paths are the open parts of $\xi(\gamma)$. They, together with $\gamma$, prevent the occurrence of closed vertices below the lowest point of $\gamma$ with two closed arms to the top side of $B(2 b N)$

where

$$
B_{l, k}:=[-a N, a N] \otimes\left(\left(2 \frac{l}{k}-1\right) a N,\left(2 \frac{l+1}{k}-1\right) a N\right] .
$$

Let $\lambda_{1}, \lambda_{2} \in \mathbb{R}$. Then there exist $C=C\left(a, b, \lambda_{1}, \lambda_{2}\right)$ and $N_{0}=N_{0}\left(a, b, \lambda_{1}, \lambda_{2}, k\right)$ such that for all $\lambda \in\left[\lambda_{1}, \lambda_{2}\right]$ and $k, l \in \mathbb{N}$ with $l \leq k-1$ we have

$$
\mathbb{P}_{p_{\lambda}(N)}\left(L_{l, k}(R, r)\right) \leq C \mathbb{P}_{p_{\lambda}(N)}\left(L_{l, k}(B(2 b N), \text { top }(B(2 b N)))\right)
$$

for $N \geq N_{0}$.

Proof of Lemma 5.6 The proof follows the arguments of the proof of Lemma 5.3. Our aim is to show that, conditioning on $L_{l, k}(R, r)$, the open and closed crossings of Fig. 2 occur with probability bounded away from 0 .

Let $s_{L}\left(s_{R}\right.$, resp.) denote the starting (ending, resp.) vertex of $r$. We say that a path $\rho \subseteq R \cup r$ is good, if it

- starts at $s_{L}$ and ends at $s_{R}$,

- it is non-self touching, and

- one of its lowest points is in $B_{l, k}$.

Let $\rho$ be a fixed good path. Let $B e(\rho, R)$ denote the set of vertices in $R$ 'under' $\rho$. It is the intersection of $R$ with the connected component of $\partial R \backslash r$ in $c l(R) \backslash \rho$. Let $A b(\rho, R):=R \backslash B e(\rho, R)$. Recall Definition 5.2.

Let $O_{\rho}$ denote the event that there is path $v$ such that

- $v$ is non self-touching,

- $v \subseteq B_{0}:=[-2 b N, 2 b N] \otimes[-a N, 2 a N]$,

- $v$ connects the left and the right side of the parallelogram $B_{1}:=[-2 b N, 2 b N] \otimes$ $[a N, 2 a N]$, 
- $v \backslash R \subset B_{1}$ and the vertices in $v \backslash R$ are open, and

- each of the paths of $v \cap R$ is a concatenation of some open paths which lie in $B e(\rho, B(b N)) \cap B_{1}$, and of some subpaths of $\rho$.

Let $\gamma$ denote the lowest non-self touching path in $R \cup r$ which starts at $s_{L}$ and ends at $s_{R}$, and of which all the vertices outside of $r$ are closed. Note that on the event $L_{l, k}(R, r)$, $\gamma$ is good. By simple modifications of the arguments in the proof of Lemma 5.3 we get that there are $c_{1}=c_{1}\left(a, b, \lambda_{1}, \lambda_{2}\right)>0$ and $N_{1}=N_{1}\left(a, b, \lambda_{1}, \lambda_{2}\right)$ such that

$$
\mathbb{P}_{p_{\lambda}(N)}\left(L_{l, k}(R, r) \cap O_{\gamma}\right) \geq c_{1} \mathbb{P}_{p_{\lambda}(N)}\left(L_{l, k}(R, r)\right)
$$

for $l, k \in \mathbb{N}, 0 \leq l \leq k-1, \lambda \in\left[\lambda_{1}, \lambda_{2}\right]$ for $N \geq N_{1}$.

Recall Definition 5.2. Let $\zeta \in \operatorname{HCr}(B(2 b N))$. On the event $L_{l, k}(R, r) \cap$ $O_{\gamma}$ we have $R \cap(\mathbb{Z} \otimes[3 a N, b N]) \subset A b(\xi(\gamma), B(2 b N))$. Hence the event $L_{l, k}(R, r) \cap O_{\gamma} \cap\{\xi(\gamma)=\zeta\}$ is decreasing on the configuration in $A b(\zeta, B(2 b N))$. Let $B_{2}=[-2 b N, 2 b N] \otimes[3 a N, 4 a N], B_{3}=[-2 b N,-b N] \otimes[3 a N, 2 b N]$, $B_{4}=[b N, 2 b N] \otimes[3 a N, 2 b N]$ and $\mathcal{D}=\mathcal{H}_{c}\left(B_{2}\right) \cap \mathcal{V}_{c}\left(B_{3}\right) \cap \mathcal{V}_{c}\left(B_{4}\right)$. The arguments of the proof of Lemma 5.3 give that there exist $c_{2}=c_{2}\left(a, b, \lambda_{1}, \lambda_{2}\right)>0$ and $N_{2}=N_{2}\left(a, b, \lambda_{1}, \lambda_{2}, k\right)$ such that

$$
\mathbb{P}_{p_{\lambda}(N)}\left(L_{l, k}(R, r) \cap O_{\gamma} \cap \mathcal{D}\right) \geq c_{2} \mathbb{P}_{p_{\lambda}(N)}\left(L_{l, k}(R, r) \cap O_{\gamma}\right)
$$

for $l, k \in \mathbb{N}, 0 \leq l \leq k-1, \lambda \in\left[\lambda_{1}, \lambda_{2}\right]$ for $N \geq N_{2}$. Note that $L_{l, k}(R, r) \cap O_{\gamma} \cap \mathcal{D} \subset$ $L_{l, k}(B(2 b N)$, top $(2 b N))$. See Fig. 2 for more details. This combined with (5.11) and (5.12) finishes the proof of Lemma 5.6.

A combination of Lemma 5.3 and 5.6 gives the following:

Corollary 5.7 Suppose that the conditions of Lemma 5.3 hold. Then there exist $c=$ $c\left(a, b, \lambda_{1}, \lambda_{2}\right)$ and $N_{0}=N_{0}\left(a, b, \lambda_{1}, \lambda_{2}, k\right)$ such that

$$
\mathbb{P}_{p_{\lambda}(N)}\left(L_{l, k}(R, r)\right) \leq c k^{-1}
$$

for $l=0,1, \ldots, k-1, \lambda \in\left[\lambda_{1}, \lambda_{2}\right]$ and $N \geq N_{0}$.

5.3 The diameter of the active clusters close to time $1 / 2$

We turn to the $N$-parameter frozen percolation process. In the introduction we indicated that the $N$-parameter frozen percolation process exists since it is a finite range interacting particle system. It is also true that the process is measurable with respect to the $\tau$ values.

Definition 5.8 For $t \in[0,1]$ and $J \subset V$ let

$$
\mathcal{F}_{t}(J):=\sigma\left(\left\{\tau_{w}<s\right\} \mid w \in J, s \in[0,1]\right)
$$

denote the $\sigma$-algebra generated by the $\tau$ values of the vertices in $J$ up to time $t$. 
The following lemma follows from the arguments in the second lecture of [11].

Lemma 5.9 For $N \in \mathbb{N}$, the $N$-parameter frozen percolation process is adapted to the filtration $\mathcal{F}_{t}(V)$.

Recall the notation $\mathcal{C}_{a}(v ; \lambda)$ from Definition 3.4. We prove the following proposition.

Proposition 3.5 For all $\lambda \in \mathbb{R}$ and $\varepsilon, K, \alpha>0$, there exist $\theta=\theta(\lambda, \alpha, \varepsilon, K)>0$ and $N_{0}=N_{0}(\lambda, \alpha, \varepsilon, K)$ such that

$$
\mathbb{P}_{N}\left(\exists v \in B(K N) \text { s.t. } \operatorname{diam}\left(\mathcal{C}_{a}(v ; \lambda)\right) \in((\alpha-\theta) N,(\alpha+\theta) N)\right)<\varepsilon
$$

for $N \geq N_{0}$.

Proof of Proposition 3.5 Due to the length of the proof, we first give an outline. Let $\lambda, \varepsilon, K, \alpha$ as in the statement of Proposition 3.5.

For simplicity, we only give a sketch which shows that we can choose $\theta \in\left(0, \frac{1 \wedge \alpha}{2}\right)$ such that

$$
\mathbb{P}_{N}\left(\operatorname{diam}\left(\mathcal{C}_{a}(\lambda)\right) \in((\alpha-\theta) N,(\alpha+\theta) N)\right)<\varepsilon
$$

for large $N$.

Let us denote by $\tilde{x}, \tilde{y}$ a pair of sites in the active cluster of the origin for which $d(\tilde{x}, \tilde{y})=\operatorname{diam}\left(\mathcal{C}_{a}(\lambda)\right)$. We consider the case where $\tilde{x}$ is one of the lowest and $\tilde{y}$ is one of the highest vertices of the active cluster. The other case where the diameter is achieved as a distance between a leftmost and rightmost vertex can be treated in a similar way. Let $x$ ( $y$, resp.) denote a vertex which is a neighbour of $\tilde{x}(\tilde{y}$, resp.), and lies below (above, resp.) it. Note that $x$ and $y$ are closed frozen vertices at time $p_{\lambda}(N)$.

In Step 1 we apply Observation 3.2 and Lemma 2.27 to set $\lambda_{0}$ so that with probability close to 1 , there are no frozen clusters at time $p_{\lambda_{0}}(N)$ in $B((\alpha+2) N)$. Hence in the case where $\lambda_{0} \geq \lambda$ the statement of Proposition 3.5 follows. In the following we assume that $\lambda_{0}<\lambda$, and the event in (5.13) is non-empty. We investigate the configuration close to $x$. In Step 2, we show that with probability close to 1 , there is a unique frozen cluster $F$ close to $x$. By Step 1, we can assume that it froze at time $p_{\lambda_{F}}(N)$ for $\lambda_{F} \in\left[\lambda_{0}, \lambda\right]$. In Step 4, we show that with probability close to 1 , there is a graph $\mathcal{R} \subseteq \mathbb{T}$ such that its boundary consists of a $p_{\lambda_{F}}(N)$-closed arc, denoted by $r_{c}$, and a $p_{\lambda_{F}}(N)$-open arc. In Steps 3,5 and 6 we show that with probability close to 1 , we can impose some extra conditions on $\mathcal{R}$ and $r_{c}$ and on the configuration in $\mathcal{R}$. We get a pair $\left(\mathcal{R}, r_{c}\right)$ with the following properties:

- $\partial \mathcal{R}$ is a certain outermost circuit, which is measurable with respect to the $\tau$-values in $\mathbb{T} \backslash \mathcal{R}$, (Step 4)

- $x$ is one of the lowest vertices of $\mathcal{R}$ with two non-touching $p_{\lambda_{F}}(N)$-closed arms in $\mathcal{R}$ to $r_{c}$, (Step 4)

- no matter how we change the $\tau$ values in $\mathcal{R}$, the $N$-parameter frozen percolation outside $\mathcal{R}$ does not change up to time $p_{\lambda}(N)$, (Step 3)

- satisfies a technical condition (Step 5) 
- $y \in \mathbb{T} \backslash c l(\mathcal{R})($ Step 4).

Let us condition on the $\tau$-values in $\mathbb{T} \backslash \mathcal{R}$. The first and the third property of $\left(\mathcal{R}, r_{c}\right)$ implies that at time $p_{\lambda_{F}}(N)$, the vertices in $\mathcal{R}$ are open with probability $p_{\lambda_{F}}(N)$ and closed with probability $1-p_{\lambda_{F}}(N)$ independently from each other. This combined with $y \in \mathbb{T} \backslash \mathcal{R}$ allows us to decouple the locations of $x$ and $y$. Since $d(\tilde{x}, \tilde{y})=$ $\operatorname{diam}\left(\mathcal{C}_{a}(\lambda)\right)$, to prove (5.14), it is enough to show that the second coordinate of $x$ is not concentrated when we condition on the configuration in $\mathbb{T} \backslash \mathcal{R}$. We would like to use Corollary 5.7 for the pair $\left(\mathcal{R}, r_{c}\right)$. Unfortunately, this pair $\left(\mathcal{R}, r_{c}\right)$ might not satisfy all the conditions of Definition 5.5. To solve this problem we use the technical condition of Step 5 and we construct the pair $\left(\tilde{\mathcal{R}}, \tilde{r}_{c}\right)$ from $\left(\mathcal{R}, r_{c}\right)$ using a deterministic procedure in Step 6 such that

- $\tilde{\mathcal{R}} \subset \mathcal{R}$,

- a translated version of $\left(\tilde{\mathcal{R}}, \tilde{r}_{c}\right)$ is $\left(\alpha_{3} N, \alpha_{2} N\right)$-regular as of Definition 5.5 for some $\alpha_{2}, \alpha_{3}>0$, and

- $x$ is one of the lowest vertices of $\tilde{\mathcal{R}}$ with two non-touching $p_{\lambda_{F}}(N)$-closed arms in $\tilde{\mathcal{R}}$ to $\tilde{r}_{c}$.

We apply Corollary 5.7 to $\left(\tilde{\mathcal{R}}, \tilde{r}_{c}\right)$ and get the required deconcentration result and finish the proof of Proposition 3.5. We make this argument precise in Step 7.

Remark The structure of the proofs in Steps 2-6 is an arm event hunting procedure. We take a some small neighbourhood of $x$. We deduce that if the required condition is violated, then certain mixed near-critical arm events or crossing events of thin parallelograms occur. These events have upper bounds with exponents strictly larger than 2 . This implies that by choosing the neighbourhood small enough, we can set their probability as small as we want. In particular, we get that the probability of the event where the condition of the step is not satisfied is as small as required, and finishes the proof of the step.

Let us turn to the precise proof.

Step 1. We set $\lambda_{0}$ such that with probability close to 1 , at time $p_{\lambda_{0}}(N)$, none of the open clusters intersecting $B((2 \alpha+K+2) N)$ are frozen.

By Lemma 2.27 we choose $\lambda_{0}=\lambda_{0}(\alpha, \varepsilon, K)$ and $N_{0}=N_{0}(\alpha, \varepsilon, K)$ such that the event

$$
E_{0}:=\mathcal{N}_{c}\left(\lambda_{0}, 1 / 24,2 \alpha+K+4, N\right)
$$

has probability at least $1-\varepsilon / 20$ for $N \geq N_{0}$. Then by Observation 3.2 we have that none of open clusters intersecting $B((2 \alpha+K+2) N)$ are frozen. In particular, if a vertex $v \in B((2 \alpha+K+2) N)$ is closed at time $p_{\lambda}(N)$, then it is $p_{\lambda_{0}}(N)$-closed. Moreover, if $v \in B((2 \alpha+K+2) N)$ is open at time $p_{\lambda}(N)$, then it is $p_{\lambda}(N)$-open. This finishes Step 1.

Remark Note that in the definition of $E_{0}$ above, we set the second argument of $\mathcal{N}_{c}$ to $1 / 24$, which is smaller than $1 / 6$ which appears in Observation 3.2. The reason for this choice will become clear in Step 3. 


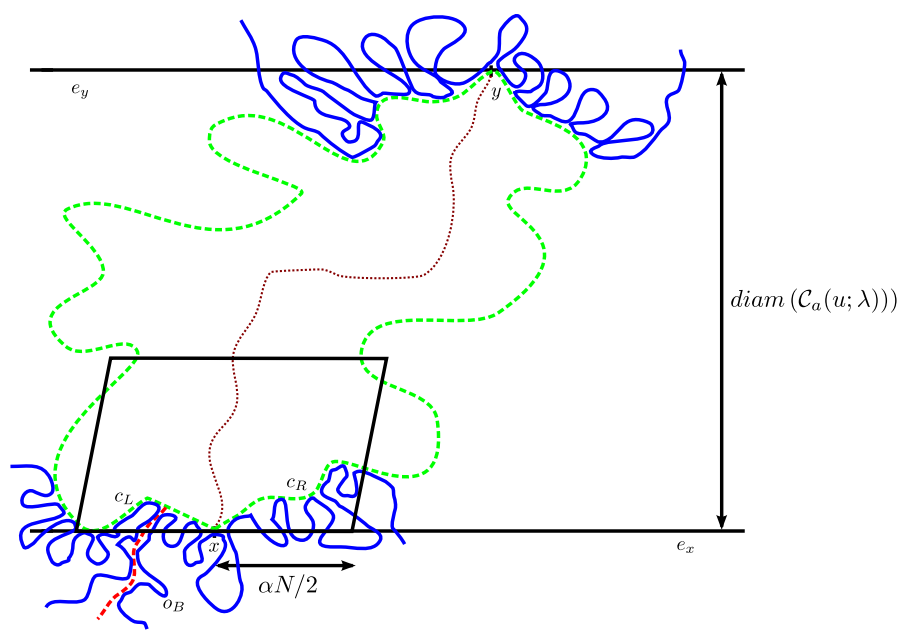

Fig. 3 The closed boundary of $\mathcal{C}_{a}(\lambda)$ gives rise to the closed arms $c_{L}$ and $c_{R}$ from $x$ to $\partial B(x ; \alpha N / 2)$. The frozen vertex neighbouring $x$ provides the arm $o_{B}$

Let $\theta \in\left(0, \frac{1 \wedge \alpha}{2}\right)$. For $i=1,2$, let $B A^{i}=B A^{i}(\theta)$ denote the set of vertices $v \in B(K N)$ such that there are $\tilde{x}(v)=\left(\tilde{x}_{1}(v), \tilde{x}_{2}(v)\right), \tilde{y}(v)=\left(\tilde{y}_{1}(v), \tilde{y}_{2}(v)\right) \in$ $\mathcal{C}_{a}(v ; \lambda)$ such that

$$
\tilde{y}_{i}(v)-\tilde{x}_{i}(v)=d(\tilde{x}(v), \tilde{y}(v))=\operatorname{diam}\left(\mathcal{C}_{a}(v ; \lambda)\right) \in((\alpha-\theta) N,(\alpha+\theta) N) .
$$

Note that

$\left\{\exists v \in B(K N)\right.$ s.t. $\left.\operatorname{diam}\left(\mathcal{C}_{a}(v ; \lambda)\right) \in((\alpha-\theta) N,(\alpha+\theta) N)\right\}=\left\{B A^{1} \cup B A^{2} \neq \emptyset\right\}$.

Let $u \in B A^{2}$. In the following we define quantities which depend on the value of $u$. In notation we only indicate the dependence on $u$ in the first appearance of these quantities, or when we want to emphasize this dependence. For each $u \in B A^{2}$ we fix a pair $(\tilde{x}, \tilde{y})=(\tilde{x}, \tilde{y})(u)$ which satisfies (5.16). It can happen that there are more than one candidates for $\tilde{x}$ or $\tilde{y}$. In this case we choose one of them in some deterministic way. (E.g we can set $\tilde{x}$ and $\tilde{y}$ as the leftmost vertex among the candidates.) Let $x=x(u)(y(u)$, resp.) denote a (deterministically chosen) neighbour of $\tilde{x}$ ( $\tilde{y}$, resp.) below $\tilde{x}$ (above $\tilde{y}$, resp.). The active cluster $\mathcal{C}_{a}(u ; \lambda)$ lies between the horizontal lines passing through $x$ and $y$ denoted by $e_{x}$ and $e_{y}$. Since $\theta<\alpha / 2$, the outer boundary of $\mathcal{C}_{a}(u ; \lambda)$ provides two non-touching closed half plane arms in $x+\mathbb{Z} \otimes[0, \infty)$ to distance $\alpha N / 2$ starting from $x$. Since $\partial \mathcal{C}_{a}(u ; \lambda) \subset B((2 \alpha+K+2) N)$, by Step 1, on the event $E_{0}$ these arms are $p_{\lambda_{0}}(N)$-closed. We denote the one on the left (right, resp.) hand side by $c_{L}=c_{L}(u)$ $\left(c_{R}=c_{r}(u)\right.$, resp.). Apart from their common starting point, $c_{L}$ and $c_{R}$ do not even touch, since any active path connecting $\tilde{x}$ to $\tilde{y}$ separates them. Since $x$ is a closed frozen vertex, there is at least one open frozen neighbour of $x$. From this vertex there is a $p_{\lambda}(N)$-open arm $o_{B}=o_{B}(u)$ to distance at least $N / 2$. See Fig. 3 for more details. 
Let $\beta, \beta^{\prime} \in(0,1)$ with $\beta<\beta^{\prime}$. Recall the definition of the events $\mathcal{N} \mathcal{A}\left(\beta, \beta^{\prime}\right):=$ $\mathcal{N} \mathcal{A}\left(\beta, \beta^{\prime}, \lambda, \lambda_{0}, 2 \alpha+K+2, N\right)$ and $\mathcal{N C}\left(\beta, \beta^{\prime}\right):=\mathcal{N C}\left(\beta, \beta^{\prime}, \lambda, \lambda_{0}, 2 \alpha+K+2\right.$, $N)$ from Corollary 2.13 and 2.22. In the following we introduce the constants $\alpha_{i}>0$ for $i=1,2,3$ such that $\alpha_{i} / \alpha_{i+1} \gg 1$. Let $\alpha_{3} \in\left(0, \frac{\alpha \wedge 1}{2}\right)$. Let $z=z(u) \in V$ such that $x=x(u) \in\left[-\alpha_{3} N, \alpha_{3} N\right] \otimes\left(-\alpha_{3} N, \alpha_{3} N\right]+\left\lfloor\alpha_{3} N\right\rfloor z$. Note that $z \in B\left(\left\lceil\frac{2 \alpha+K+2}{\alpha_{3}}\right\rceil\right)$. We define $B_{3}=B_{3}(u):=B\left(\left\lfloor\alpha_{3} N\right\rfloor z ; \alpha_{3} N\right)$. Note that throughout the arguments below, we will assume that $\alpha_{1}>\alpha_{2}>\alpha_{3}$, however, we will set their precise values only in later stages of the proof.

Step 2. We show that with probability close to 1, there is only one frozen cluster close to $x=x(u)$ for all $u \in B A^{2}$.

Let $\alpha_{1} \in\left(0, \frac{\alpha \wedge 1}{2}\right), B_{1}=B_{1}(u):=B\left(\left\lfloor\alpha_{3} N\right\rfloor z ; \alpha_{1} N\right)$ and $A_{1}=A_{1}(u):=$ $A\left(\left\lfloor\alpha_{3} N\right\rfloor z ; \alpha_{1} N, \frac{\alpha \wedge 1}{2} N\right)$. Suppose that there are at least two different frozen clusters in $B_{1}$. On the event $E_{0}$ we find 5,2 mixed near critical arms in $A_{1}:$ the two $p_{\lambda_{0}}(N)$ closed arms $c_{L}$ and $c_{R}$, the two $p_{\lambda}(N)$-open arms from the two frozen clusters, and a $p_{\lambda_{0}}(N)$-closed arm separating them. Let $E_{1}:=\mathcal{N} \mathcal{A}\left(\alpha_{1}, \frac{\alpha \wedge 1}{2}\right)$. Hence we get:

Claim 5.10 On the event $E_{0} \cap E_{1}, \forall u \in B A^{2}$, there is a unique frozen cluster denoted by $F=F(u)$ which intersects $B_{1}(u)$. Let $\lambda_{F}=\lambda_{F}(u) \in\left[\lambda_{0}, \lambda\right]$ such that $F$ froze at $p_{\lambda_{F}}(N)$. On $E_{0} \cap E_{1}$, a vertex in $B_{1}(u)$ is open in the $N$-parameter frozen percolation process at time $p_{\lambda_{F}}(N)$ if and only if it is $p_{\lambda_{F}}(N)$-open.

In the following two steps we write open (closed, resp.) for $p_{\lambda_{F}}(N)$-open $\left(p_{\lambda_{F}}(N)\right.$ closed, resp.) if it is not stated otherwise. We finish Step 2 by applying Corollary 2.13 and we set $\alpha_{1}$ such that

$$
\mathbb{P}\left(E_{1}\right) \geq 1-\varepsilon / 20
$$

for $N \geq N_{1}\left(\varepsilon, \lambda_{0}, \lambda, K\right)$.

Step 3. We say that a circuit is $p_{\lambda_{F}}(N)$-open-closed, or simply open-closed, if it consists of a $p_{\lambda_{F}}(N)$-open and a $p_{\lambda_{F}}(N)$-closed arc. Suppose that there is a $p_{\lambda_{F}}(N)$ open-closed circuit close to and around $x$. We show that with probability close to 1 , no matter how we change the $\tau$ values inside this circuit, the $N$-parameter frozen percolation process does not change till time $p_{\lambda}(N)$ outside of the circuit.

Let $\alpha_{2} \in\left(0, \alpha_{1} \wedge \frac{1}{4}\right)$, and $\beta_{2} \in\left(\alpha_{2}, \alpha_{1}\right)$ be some intermediate scale. We define the parallelograms

$$
\begin{aligned}
& B_{2}=B_{2}(u):=B\left(\left\lfloor\alpha_{3} N\right\rfloor z ; \alpha_{2} N\right), \\
& B_{2}^{\prime}=B_{2}^{\prime}(u):=B\left(\left\lfloor\alpha_{3} N\right\rfloor z ; \beta_{2} N\right), \\
& A_{2}=A_{2}(u):=A\left(\left\lfloor\alpha_{3} N\right\rfloor z ; \alpha_{2} N, \alpha_{1} N\right), \\
& A_{2}^{\prime}=A_{2}^{\prime}(u):=A\left(\left\lfloor\alpha_{3} N\right\rfloor z ; \beta_{2} N, \alpha_{1} N\right) .
\end{aligned}
$$

Let $B L=B L(u)$ denote the set of bordering lines of $F \backslash B_{2}^{\prime}$, that is the top-and bottom-most horizontal, left- and rightmost vertical lines which intersect $F \backslash B_{2}^{\prime}$. We rule out the case where there is a line in $B L$ which intersects $B_{2}^{\prime}$ in the following technical claim. 
Claim 5.11 Let

$$
E_{2}^{\prime}=\mathcal{N} \mathcal{A}\left(2 \beta_{2}, \alpha_{1}-2 \beta_{2}\right) \cap \mathcal{N C}\left(2 \beta_{2}, 2 \alpha_{1}\right)
$$

Then

$E_{0} \cap E_{1} \cap E_{2}^{\prime} \subset E_{0} \cap E_{1} \cap\left\{\forall u \in B A^{2}, \forall e \in B L(u)\right.$ we have $\left.e \cap\left(F \backslash B_{2}^{\prime}\right)=\emptyset\right\}$.

Proof of Claim 5.11 Let $u \in B A^{2}$. When the bottom-most line of $F \backslash B_{2}^{\prime}$ intersects $B_{2}^{\prime}$, then $F \subseteq\left(\mathbb{Z} \otimes\left[-\beta_{2} N, \infty\right)\right)+\left\lfloor\alpha_{3} N\right\rfloor z$. We see 4 half plane arms: $c_{L}, c_{R}$ give two closed and $o_{B}$ gives an open arm, a fourth closed half plane arm separates $F$ from the line $\mathbb{Z} \otimes\left\{\left\lfloor\beta_{2} N\right\rfloor\right\}+\left\lfloor\alpha_{3} N\right\rfloor z$. Hence $\mathcal{N} \mathcal{A}^{c}\left(2 \beta_{2}, \alpha_{1}-2 \beta_{2}\right)$ occurs.

If the topmost line of $F \backslash B_{2}^{\prime}$ intersects $B_{2}^{\prime}$, then the closed arms $c_{L}$ and $c_{R}$ stay in the parallelogram

$$
\left[-\alpha_{1} N, \alpha_{1} N\right] \otimes\left[-\beta_{2} N, \beta_{2} N\right]+\left\lfloor\alpha_{3} N\right\rfloor z
$$

In particular, $c_{L}$ gives a closed crossing of one of the parallelograms

$$
\begin{gathered}
{\left[-\alpha_{1} N,-\beta_{2} N\right] \otimes\left[-\beta_{2} N, \beta_{2} N\right]+\left\lfloor\alpha_{3} N\right\rfloor z \text { or }} \\
{\left[\beta_{2} N, \alpha_{1} N\right] \otimes\left[-\beta_{2} N, \beta_{2} N\right]+\left\lfloor\alpha_{3} N\right\rfloor z .}
\end{gathered}
$$

That is, the event $\mathcal{N} \mathcal{C}^{c}\left(2 \beta_{2}, 2 \alpha_{1}-2 \beta_{2}\right)$ occurs.

When a leftmost bordering line of $F \backslash B_{2}^{\prime}$ intersects $B_{2}^{\prime}$, then we find that the arms in $A\left(\left\lfloor\alpha_{3} N\right\rfloor z ; \beta_{2} N, \alpha_{1} N\right)$ induced by $c_{L}, c_{R}$ and $o_{B}$ stay in half plane

$$
\left[-2 \beta_{2} N, \infty\right) \times \mathbb{R}+\left\lfloor\alpha_{3} N\right\rfloor z
$$

The frozen cluster $F$ is separated from the line $\left\{-2 \beta_{2} N\right\} \times \mathbb{R}+\left\lfloor\alpha_{3} N\right\rfloor z$. This provides an additional closed arm in the half plane (5.20), which together the arms induced by $c_{L}, c_{R}$ and $o_{B}$ give 4 half plane arms, hence the event $\mathcal{N} \mathcal{A}^{c}\left(\beta_{2}, \alpha_{1}-2 \beta_{2}\right)$ occurs.

The case when the rightmost bordering line of $F \backslash B_{2}^{\prime}$ intersects $B_{2}^{\prime}$ can be treated similarly.

With the notation (5.19) we get that on the event $E_{0} \cap E_{1} \cap E_{2}^{\prime}$, none of the lines of $B L$ intersect $B_{2}^{\prime}$, which finishes the proof of Claim 5.11.

Now we proceed with Step 3. Let $u \in B A^{2}$. Suppose that there is an open-closed circuit $O C=O C(u)$ around $x$ in $B_{2}$. Let $I=I(u)$ denote the union of the finite connected components of $\mathbb{T} \backslash O C$. Let us change the $\tau$ values of the vertices in $I$ in some arbitrary non-degenerate way (that is, the new $\tau$ values are all different), but keep the original values outside $I$. Let us run the $N$-parameter frozen percolation dynamics for this modified set of $\tau$ values. We denote this new process by $F P P^{\prime}$ and $F P P$ denotes the original process. Our next aim is to show that the processes $F P P$ and $F P P^{\prime}$ coincide on $V \backslash B_{2}$ till time $p_{\lambda}(N)$ on some event $E_{2}$ independently from the choice of the new $\tau$ values.

Recall the definition of $E_{0}$ from (5.15) and the remark after Step 1. Since $\alpha_{2}<\alpha_{1}<$ $1 / 24$ and $I \subseteq B_{2}$, the definition of $E_{0}$ and Observation 3.2 give that the processes 
$F P P$ and $F P P^{\prime}$ coincide on $V \backslash I$ up to time $p_{\lambda_{0}}(N)$. In particular, the closed arc of $O C$ stays closed till time $p_{\lambda_{F}}(N)$ in both processes. Hence it acts as a barrier for the effect of $\tau$ values in $I$. By Step 2, the open arc of $O C$ is a subset of $F$.

Case 1. The process $F P P^{\prime}$ differs from $F P P$ outside of $R$ at some time $t \in$ $\left[0, p_{\lambda_{F}}(N)\right]$.

By Claim 5.11 on the event $E_{0} \cap E_{1} \cap E_{2}^{\prime}$ if these two processes differ outside $I$, then in the process $F P P^{\prime}$ a frozen cluster $F^{\prime}$ emerged before time $p_{\lambda_{F}}(N)$ such that $F^{\prime} \backslash I \neq F \backslash I$. By the arguments above, we get that $F^{\prime}$ froze in at time $p_{\lambda_{F^{\prime}}}(N)$ with $\lambda_{F^{\prime}} \in\left[\lambda_{0}, \lambda_{F}\right]$. Let $B L^{\prime}$ denote the set of bordering lines of $F^{\prime} \backslash B_{2}^{\prime}$. With careful examination of the proof of Claim 5.11 one can see that the arguments applied there can also be applied to the new process $F P P^{\prime}$. We get that, on the event $E_{0} \cap E_{1} \cap E_{2}^{\prime}$ none of the lines of $B L^{\prime}$ intersect $B_{2}^{\prime}$ no matter how we modify the $\tau$ values in $I$. This implies that $F^{\prime} \backslash I$ has two connected components $F_{1}^{\prime}$ and $F_{2}^{\prime}$ such that $\operatorname{diam}\left(F_{i}^{\prime}\right)<N$ for $i \in\{1,2\}$, but $\operatorname{diam}\left(F_{1}^{\prime} \cup F_{2}^{\prime}\right) \geq N$. Since $I \subset B_{2}$, each of $F_{1}^{\prime}, F_{2}^{\prime}$ contains a $p_{\lambda_{F^{\prime}}}(N)$-open arm in the annulus $A_{2}^{\prime \prime}=A_{2}^{\prime \prime}(u):=A\left(\left\lfloor\alpha_{3} N\right\rfloor z ; \alpha_{2} N, \beta_{2} N\right)$. When for some $i \in\{1,2\} F_{i}^{\prime}$ lies above $c_{L}$ and $c_{R}$, then we get a 4, 3 near critical arm event: the closed arms induced by $c_{L}, c_{R}$ and the open arm induced by $F_{i}^{\prime}$ stay above $e_{x}$, and $o_{B}$ provides the fourth arm in $A_{2}^{\prime \prime}$. Hence $\mathcal{N} \mathcal{A}^{c}\left(\alpha_{2}, \beta_{2}\right)$ occurs. If both of $F_{1}^{\prime}, F_{2}^{\prime}$ lie below $c_{L}$ and $c_{R}$ then we get a 5,2 near critical mixed arm event in $A_{2}^{\prime \prime}: c_{L}, c_{R}$ induce closed half plane arms in $A_{2}^{\prime \prime} . F_{1}^{\prime}, F_{2}^{\prime}$ induce two open arms. Since $F_{1}^{\prime}$ and $F_{2}^{\prime}$ are different connected components of $F^{\prime} \backslash I$, there is a fifth, $p_{\lambda_{F^{\prime}}}(N)$-closed, arm separating $F_{1}^{\prime}$ and $F_{2}^{\prime}$ in $A_{2}^{\prime \prime}$. Hence $\mathcal{N} \mathcal{A}^{c}\left(\alpha_{2}, \beta_{2}\right)$ occurs. Let $E_{2}=E_{2}^{\prime} \cap \mathcal{N} \mathcal{A}\left(\alpha_{2}, \beta_{2}\right)$.

Case 2. FPP and $F P P^{\prime}$ coincide on $V \backslash I$ till $p_{\lambda_{F}}(N)$, but differ outside of $R$ at some time $t \in\left(p_{\lambda_{F}}(N), p_{\lambda}(N)\right]$.

By Claim 5.11 and from that the two processes coincide outside of $R$, we get that a frozen cluster $F^{\prime}$ is formed at time $p_{\lambda_{F}}(N)$ in the new process. Moreover, $F^{\prime} \backslash I=F \backslash I$. However, the two processes differ at some time $t \in\left(p_{\lambda_{F}}(N), p_{\lambda}(N)\right]$, hence an additional frozen cluster $F^{\prime \prime}$ has to emerge in this time period using some of the vertices in $I$. This induces the 5, 2 near critical mixed arm event of Step 2. Hence we proved the following claim.

Claim 5.12 On the event $E_{0} \cap E_{1} \cap E_{2}$, we have that $\forall u \in B A^{2}$, if there is a $p_{\lambda_{F}}(N)$ open-closed circuit around $x=x(u)$ in $B_{2}(u)$ then no matter how we change the $\tau$ values inside this circuit, the frozen percolation process outside it does not change till time $p_{\lambda}(N)$.

We finish Step 3 by applying Corollary 2.13 and 2.24: we fix the value of $\beta_{2}$ and $\alpha_{2}$ such that

$$
\mathbb{P}\left(E_{2}\right) \geq 1-\varepsilon / 20
$$

for $N \geq N_{2}\left(\varepsilon, \lambda_{0}, \lambda, \alpha, K\right)$.

Step 4. We show that with probability close to 1 , there is a $p_{\lambda_{F}}(N)$-open-closed circuit around $x$, such that the location where its colour changes in the circuit is 'far' above $x$.

Let $u \in B A^{2}$. Let $\alpha_{3} \in\left(0, \alpha_{2}\right), B_{3}=B_{3}(u):=B\left(\left\lfloor\alpha_{3} N\right\rfloor z ; \alpha_{3} N\right)$ and $A_{3}=$ $A_{3}(u):=A\left(\left\lfloor\alpha_{3} N\right\rfloor z ; \alpha_{3} N, \alpha_{2} N\right)$. Let $\delta_{3} \in\left(\alpha_{3}, \alpha_{2}\right)$ be an intermediate scale. We cut the annulus $A_{3}$ into three subannuli using two other intermediate scales $\beta_{3}, \beta_{3}^{\prime}$ with $\alpha_{3}<\delta_{3}<\beta_{3}<\beta_{3}^{\prime}<\alpha_{2}$ : 


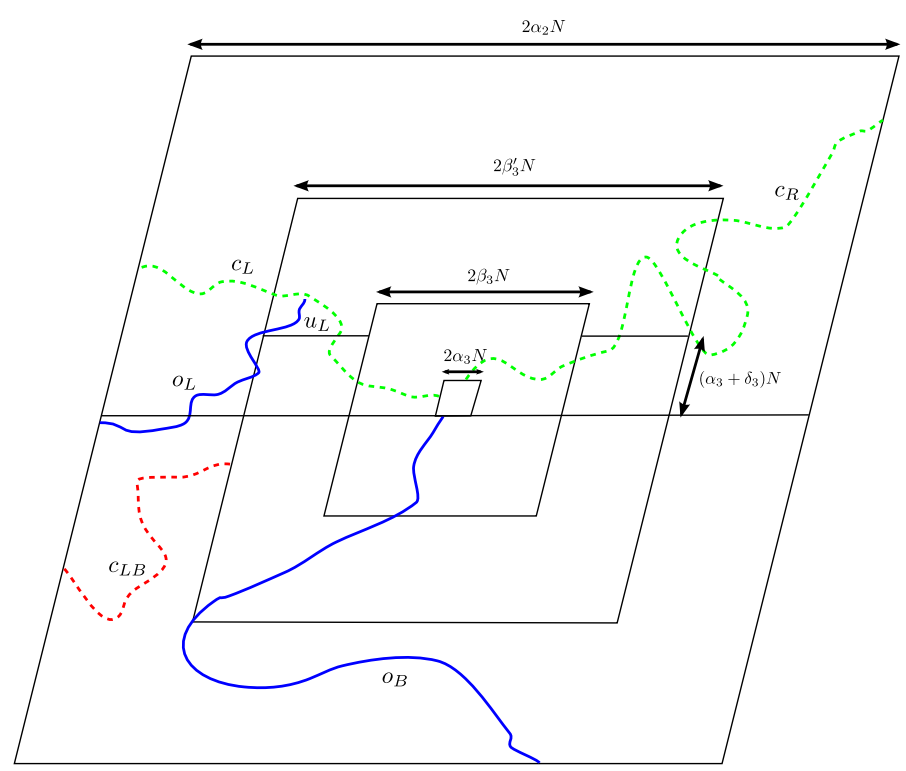

Fig. 4 The closed arm $c_{L B}$ separates $o_{L}$ and $o_{B}$ in $A_{3,2}$. Hence $c_{L}, c_{R}, o_{B}, c_{L B}, o_{L}$ give 5, 2 near critical mixed arms

$$
\begin{aligned}
& A_{3,0}=A_{3,0}(u):=A\left(\left\lfloor\alpha_{3} N\right\rfloor z ; \alpha_{3} N, \beta_{3} N\right), \\
& A_{3,1}=A_{3,1}(u):=A\left(\left\lfloor\alpha_{3} N\right\rfloor z ; \beta_{3} N, \beta_{3}^{\prime} N\right), \\
& A_{3,2}=A_{3,2}(u):=A\left(\left\lfloor\alpha_{3} N\right\rfloor z ; \beta_{3}^{\prime} N, \alpha_{2} N\right) .
\end{aligned}
$$

Let $\bar{c}_{L}\left(\bar{c}_{R}\right)$ denote the closed arm induced by $c_{L}\left(c_{R}\right)$ in $A_{3,1}$.

If $c_{L}$ and $c_{R}$ are not connected by a closed path in $A_{3,0} \cap \mathcal{C}_{a}(u ; \lambda)$, then there is a open arm separating them. Hence we see a near critical 4, 3 arm event: $c_{L}, c_{R}$ and the open arm separating them induce half plane arms in $A_{3,0}$. The fourth full plane arm in $A_{3,0}$ is induced by $o_{B}$. Thus the event $\mathcal{N} \mathcal{A}^{c}\left(\alpha_{3}, \beta_{3}\right)$ occurs.

If $\bar{c}_{L} \subseteq\left[-\beta_{3}^{\prime} N,-\beta_{3} N\right] \otimes\left[-\alpha_{3} N, \delta_{3} N\right]$ or $\bar{c}_{R} \in\left[\beta_{3} N, \beta_{3}^{\prime} N\right] \otimes\left[-\alpha_{3} N, \delta_{3} N\right]$, then we find a closed horizontal crossing in a narrow parallelogram. Hence the event $\mathcal{N C}^{c}\left(\alpha_{3}+\delta_{3}, \beta_{3}^{\prime}-\beta_{3}\right)$ occurs.

In the following we assume that both $\bar{c}_{L}$ and $\bar{c}_{R}$ leave the corresponding parallelograms. Let $w_{L}\left(w_{R}\right.$, resp.) be an open frozen vertex neighbouring a vertex of $\bar{c}_{L}\left(\bar{c}_{R}\right.$, resp.) which is outside of the aforementioned parallelogram.

Suppose that there is no open arc in $A_{3}$ connecting $w_{L}$ to $o_{B}$. Since $w_{L}$ is open frozen at time $p_{\lambda_{F}}(N)$, it has a $p_{\lambda_{F}}(N)$-open path to distance $N / 2$. Let $o_{L}$ denote the part of this path till the first time it exits $A_{3}$. Note that $o_{L}$ and $o_{B}$ are disjoint, and they are not connected by an open path inside $A_{3}$. We have two cases depending on where $o_{L}$ leaves $A_{3}$.

When it leaves $A_{3}$ by exiting its outer parallelogram, then we get a 5, 2 near critical arm event in $A_{3,2}$ : two half plane closed arms induced by $c_{L}$ and $c_{R}$, two open arms induced by $o_{L}$ and $o_{B}$ an extra closed arm separates $o_{L}$ and $o_{B}$ in $A_{3,2}$. Hence the event $\mathcal{N} \mathcal{A}^{c}\left(\beta_{3}^{\prime}, \alpha_{2}\right)$ occurs. See Fig. 4. 
Fig. 5 The outer boundary of the union of the regions surrounded by the above two open-closed circuits is open. Hence there is an open, but no open-closed, circuit surrounding both of these circuits

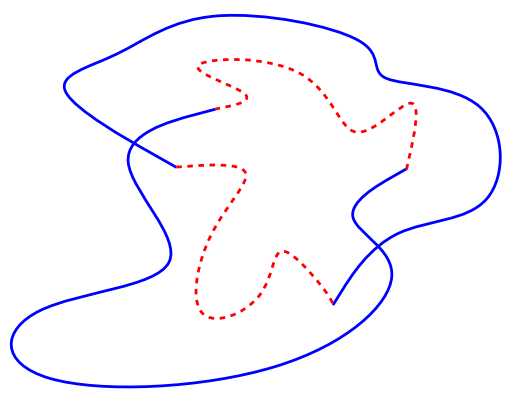

When $o_{L}$ leaves $A_{3}$ by entering its inner parallelogram, then we get a similar 5, 2 arm event in $A_{3,0}$. Thus $\mathcal{N} \mathcal{A}^{c}\left(\alpha_{3}, \beta_{3}\right)$ happens. In a similar way we can show that when $w_{R}$ is not connected to $o_{B}$ in $A_{3}$, then $\mathcal{N} \mathcal{A}^{c}\left(\beta_{3}^{\prime}, \alpha_{2}\right) \cup \mathcal{N} \mathcal{A}^{c}\left(\alpha_{3}, \beta_{3}\right)$ occurs (Fig. 5).

Let

$$
E_{3}:=\mathcal{N C}\left(\alpha_{3}+\delta_{3}, \beta_{3}^{\prime}-\beta_{3}\right) \cap \mathcal{N} \mathcal{A}\left(\alpha_{3}, \beta_{3}\right) \cap \mathcal{N} \mathcal{A}\left(\beta_{3}^{\prime}, \alpha_{2}\right) \cap \mathcal{N} \mathcal{A}\left(\alpha_{3}, \beta_{3}\right)
$$

Note that $w_{L}, w_{R} \in\left(\mathbb{Z} \otimes\left[\delta_{2} N, \alpha_{2} N\right]\right)+\left\lfloor\alpha_{3} N\right\rfloor z$, and that some parts of $c_{L}$ and $c_{R}$ are parts of the closed arc of the open-closed circuit we constructed. See Fig. 6 for more details. We arrive to the following claim.

Claim 5.13 On the event $E_{0} \cap E_{1} \cap E_{2} \cap E_{3}, \forall u \in B A^{2}$ there is a $p_{\lambda_{F}(u)}(N)$-openclosed circuit $O C=O C(u)$ with the following properties:

1. it is contained in $A_{3}(u)$ and surrounds $B_{3}(u)$,

2. the locations where the colour changes in $O C$ is contained $\left(\mathbb{Z} \otimes\left[\delta_{3} N, \alpha_{2} N\right]\right)+$ $\left\lfloor\alpha_{3} N\right\rfloor z$

3. the endpoints of the closed part of $O C$ lie in the parallelogram $\left[-\alpha_{2} N, \alpha_{2} N\right] \otimes$ $\left[\delta_{3} N, \alpha_{3} N\right]+\left\lfloor\alpha_{3} N\right\rfloor z$

4. as we walk from the outside of $B_{2}=B\left(\left\lfloor\alpha_{3} N\right\rfloor z ; \alpha_{2} N\right)$ on any of the closed arms $c_{L}$ or $c_{R}$ towards $x$, we hit the closed part of $O C$ at its endpoints for the first time.

We finish Step 4 by choosing the values of $\beta_{3}, \beta_{3}^{\prime}$ and $\delta_{3}$. The probability of $E_{3}$ is an increasing function of $\alpha_{3}$ for $\beta_{3}, \beta_{3}^{\prime}, \delta_{3}$ fixed. By Corollary 2.13 and 2.24 we choose the value of $\beta_{3}, \beta_{3}^{\prime}, \delta_{3}, \alpha_{3}$ such that the probability of the event $E_{3}$ is at least $1-\varepsilon / 20$. We only fix $\beta_{3}, \beta_{3}^{\prime}, \delta_{3}$ and require $\alpha_{3}$ to be small but unspecified so that

$$
\mathbb{P}\left(E_{3}\right) \geq 1-\varepsilon / 20
$$

for $N \geq N_{3}\left(\varepsilon, \alpha_{3}, \lambda_{0}, \lambda, \alpha, K\right)$. We choose the value of $\alpha_{3}$ in Step 6 .

In the following we will consider a certain outermost open-closed circuit. As brought to our attention by Vincent Tassion, outermost open-closed circuits are not well-defined in general: see Fig. 5. The following claim gives a sufficient condition for the existence of the outermost open-closed circuit. The proof of the claim is simple and left for the reader. 


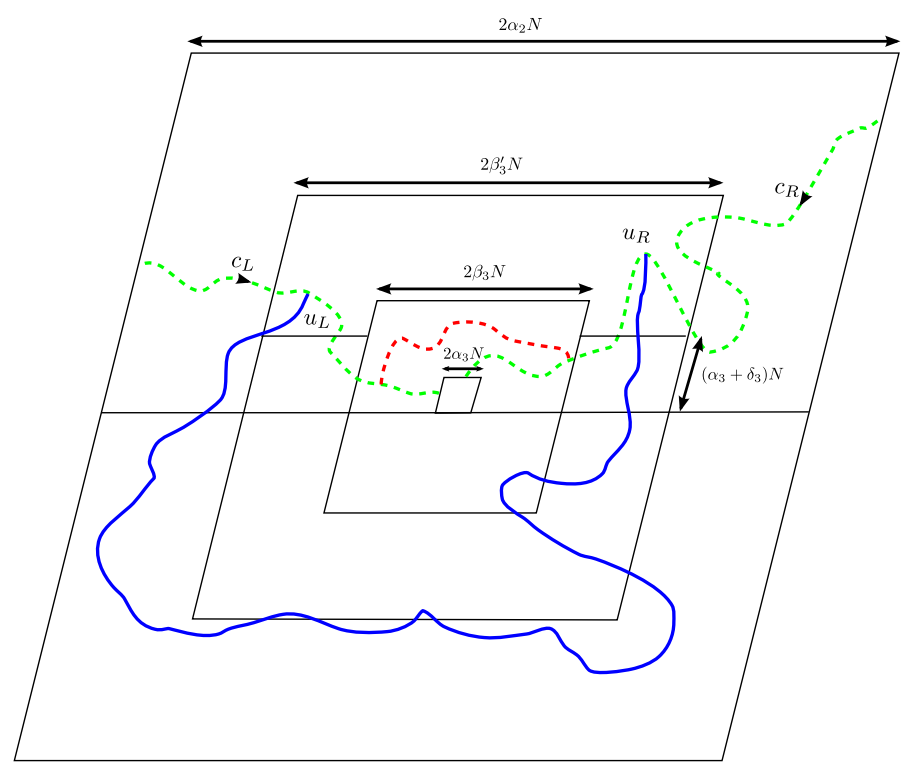

Fig. 6 The circuit around $B_{3}$ consists of the open arc drawn with continuous line subpaths of $c_{L}$ and $c_{R}$ and the closed arc in $A_{3,0}$

Claim 5.14 Let $a, b>0$ with $a<b$, and $\omega \in\{o, c\}^{A(a, b)}$. Suppose that $\omega \in \mathcal{A}_{2,(o, c)}(a, b)$ and there is an open-closed circuit in $\omega$ surrounding $B(a)$, i.e $\omega \notin \mathcal{A}_{4, a l t}(a, b)$. Then there is a unique outermost open-closed circuit surrounding $B(a)$.

Before Step 5, let us summarize what we have proved up to now. Let $u \in B A^{2}$, and suppose that the event $E_{0} \cap E_{1} \cap E_{2} \cap E_{3}\left(\alpha_{3}\right)$ holds. Using Claim 5.14, it is easy to see that the outermost open-closed circuit which satisfy the conditions of Claim 5.13 is well-defined. Let $\mathcal{O C}$ denote this outermost circuit, and $a_{c}\left(a_{o}\right.$, resp.) denote the closed (open, resp.) arcs of $\mathcal{O C}$. Further simple considerations give:

Claim 5.15 On $E_{0} \cap E_{1} \cap E_{2} \cap E_{3}\left(\alpha_{3}\right)$, for any deterministic open-closed circuit $O C$, one can check the occurrence the event $\{\mathcal{O C}=O C\}$ by looking at the $\tau$ values in the closure of the unbounded component of $V \backslash O C$.

Let $\mathcal{R}$ denote the connected component of $B_{3}$ in $\mathbb{T} \backslash \mathcal{O C}$. Let $r_{o} \subseteq a_{o}$ and $r_{c} \subseteq a_{c}$ denote the open and closed parts of $\partial \mathcal{R}$. The pair $\left(\mathcal{R}, r_{c}\right), \mathcal{O C}$ and the configuration in $\mathbb{T} \backslash \mathcal{R}$ satisfy the following conditions:

1. $\mathcal{R}$ is a connected induced subgraph of $\mathbb{T}$ (definition of $\mathcal{R}$ )

2. $B\left(\left\lfloor\alpha_{3} N\right\rfloor z ; \alpha_{3} N\right)=B_{3} \subseteq \mathcal{R} \subseteq B_{2}=B\left(\left\lfloor\alpha_{3} N\right\rfloor z ; \alpha_{2} N\right)$ (by Claim 5.13)

3. $\partial \mathcal{R}$ is disjoint union of non-empty self avoiding paths $r_{c}$ and $r_{o}$, which are oriented such that $\mathcal{R}$ lies on the right when we walk along them. We orient $a_{c}$ ( $a_{o}$, resp.) so that the orientations of $a_{c}$ and $r_{c}$ ( $a_{o}$ and $r_{o}$, resp.) are compatible.

4. $r_{c}, a_{c} \subseteq\left[-\alpha_{2} N, \alpha_{2} N\right] \otimes\left[-\alpha_{3} N, \alpha_{2} N\right]+\left\lfloor\alpha_{3} N\right\rfloor z$, (by the proof of Claim 5.13)

5. the endpoints of $a_{c}$ denoted by $s_{L}$ and $s_{R}$ lie in the parallelogram $\left[-\alpha_{2} N, \alpha_{2} N\right] \otimes$ $\left[\delta_{3} N, \alpha_{3} N\right]+\left\lfloor\alpha_{3} N\right\rfloor z$, (by Claim 5.13) 
6. when we walk along $c_{L}\left(c_{R}\right.$, resp.) towards $x$, we hit $\mathcal{O C}$ first at vertex $s_{L}\left(s_{R}\right.$, resp.), (by Claim 5.13)

7. for every vertex $v \in a_{o}$, there is a closed path in $B_{2} \backslash \mathcal{R}$ to $\partial B_{2},(\mathcal{O C}$ is outermost)

8. for every vertex $v \in a_{c}$, there is an open path in $B_{2} \backslash \mathcal{R}$ to $\partial B_{2}$ or to $\left(c_{L} \cup c_{R}\right) \backslash c l(\mathcal{R})$. $(\mathcal{O C}$ is outermost $)$.

Note that the first three conditions coincide with the first three conditions for the pair $\left(\mathcal{R}-\left\lfloor\alpha_{3} N\right\rfloor z, r_{c}-\left\lfloor\alpha_{3} N\right\rfloor z\right)$ being $\left(\alpha_{3} N, \alpha_{2} N\right)$-outer-regular of Definition 5.5. We add an extra condition in the next step.

Note that the vertex $x$ has two non-touching closed arms to $r_{c}$. Moreover, by Condition 6 above, $x$ is one of the lowest vertices in $\mathcal{R}$ with this property. With the notation of Definition 5.1 we have that $x \in \mathcal{L}\left(\mathcal{R}, r_{c}\right)$ in the $N$-parameter frozen percolation process at time $p_{\lambda_{F}}(N)$.

Step 5. Let $u \in B A^{2}$. Suppose that the event $E_{0} \cap E_{1} \cap E_{2} \cap E_{3}$ holds. Let $\mathcal{W}=\mathcal{W}(u)$ denote the connected components of $\mathcal{R} \cap\left(\mathbb{Z} \otimes\left[-\left\lfloor\alpha_{3} N\right\rfloor+1,\left\lfloor 5 \alpha_{3} N\right\rfloor-1\right]\right)$. Let $S_{\text {mid }}(\mathcal{R})$ denote the unique element of $\mathcal{W}$ which contains $B_{3}$ as a subset. We show that with probability close to $1, \partial S_{\text {mid }} \cap r_{c}=\emptyset$.

We define $e_{T}=e_{T}(u):=\left(\mathbb{Z} \otimes\left\{\left\lfloor 5 \alpha_{3} N\right\rfloor+1\right\}\right)+\left\lfloor\alpha_{3} N\right\rfloor z$ and $e_{B}=e_{B}(u):=$ $\left(\mathbb{Z} \otimes\left\{-\left\lfloor\alpha_{3} N\right\rfloor-1\right\}\right)+\left\lfloor\alpha_{3} N\right\rfloor z$. Suppose that $\partial S_{\text {mid }} \cap r_{c} \neq \varnothing$, let $w \in \partial S_{\text {mid }}(\mathcal{R}) \cap$ $r_{c} \cap e_{T}$. Consider the parallelogram $\bar{B}=B\left(w ; \delta_{3} N / 2\right)$. Let $w_{L}$ and $w_{R}$ denote the vertices of $a_{c}$ where we exit $\bar{B}$ the first time as we walk on $r_{c}$ starting from $w$ towards $s_{L}$ and $s_{R}$, resp. The part of $a_{c}$ between $w_{L}$ and $w_{R}$ cuts $\bar{B}$ into two pieces. Let $\bar{B}_{I}$ ( $\bar{B}_{E}$, resp.) denote the part which is on the right (left, resp.) hand side of $a_{c}$ when we walk from $w_{L}$ to $w_{R}$. Let $\bar{A}_{I}=\bar{B}_{I} \backslash B\left(w ; 6 \alpha_{3} N\right)$ and $\bar{A}_{E}=\bar{B}_{E} \backslash B\left(w ; 6 \alpha_{3} N\right)$. By Condition 8 above $\bar{A}_{E}$ contains an open arm. We claim that $\bar{A}_{I}$ also contains an open arm. Suppose the contrary. Then there must be a closed non self-touching arc in $\bar{A}_{I}$ preventing the occurrence of the open arm. Note that this arc is contained in $\mathcal{R}$. Then the lowest vertex of this arc has two disjoint $p_{\lambda_{F}}(N)$-closed arms to $a_{c}$, and it lies lower than $x \in B:=B\left(\left\lfloor\alpha_{3} N\right\rfloor z ; \alpha_{3} N\right)$. This contradicts $x \in \mathcal{L}\left(\mathcal{R}, r_{c}\right)$ which was shown in the lines before Step 4 . See Fig. 7. Hence $\bar{A}_{I}$ has an open arm, which together with the open arm of $\bar{A}_{E}$ and the two closed arms of $w$ provide a 4, 3 near critical mixed arm event. Hence the event $E_{4}^{c}=\mathcal{N} \mathcal{A}^{c}\left(6 \alpha_{3}, \delta_{3} / 2\right)$ occurs. Thus we arrive to the following claim and we finish Step 5.

Claim 5.16 On the event $E_{0} \cap E_{1} \cap E_{2} \cap E_{3} \cap E_{4}$, we have $\partial S_{\text {mid }}(\mathcal{R}) \cap r_{c}=\varnothing$.

Step 6. Recall Definition 5.5. We show that with probability close to 1 , we can cut down some parts of $\mathcal{R}$ and get a pair $\tilde{\mathcal{R}}$ and $\tilde{r}_{c}$ such that the pair $\left(\tilde{\mathcal{R}}-\left\lfloor\alpha_{3} N\right\rfloor z, \tilde{r}_{c}-\left\lfloor\alpha_{3} N\right\rfloor z\right)$ is $\left(\alpha_{3} N, \alpha_{2} N\right)$-regular and

$$
\mathcal{L}\left(\mathcal{R}, r_{c}\right) \cap B=\mathcal{L}\left(\tilde{\mathcal{R}}, \tilde{r}_{c}\right) \cap B
$$

Let $u \in B A^{2}$. Suppose that the event $E_{0} \cap E_{1} \cap E_{2} \cap E_{3} \cap E_{4}$ occurs. Let $\tilde{\mathcal{R}}=\tilde{\mathcal{R}}(u)$ be the connected component of $S_{\text {mid }}(\mathcal{R})$ in $\mathcal{R} \backslash \bigcup_{S \in \mathcal{W}: \partial S \cap r_{c} \neq \emptyset} c l(S)$ and $\tilde{r}_{c}=\partial \tilde{\mathcal{R}} \backslash r_{o}$. The conditions before Step 5 and Claim 5.16 gives that the pair $\left(\tilde{\mathcal{R}}-\left\lfloor\alpha_{3} N\right\rfloor z, \tilde{r}_{c}-\left\lfloor\alpha_{3} N\right\rfloor z\right)$ is $\left(\alpha_{3} N, \alpha_{2} N\right)$-regular. 


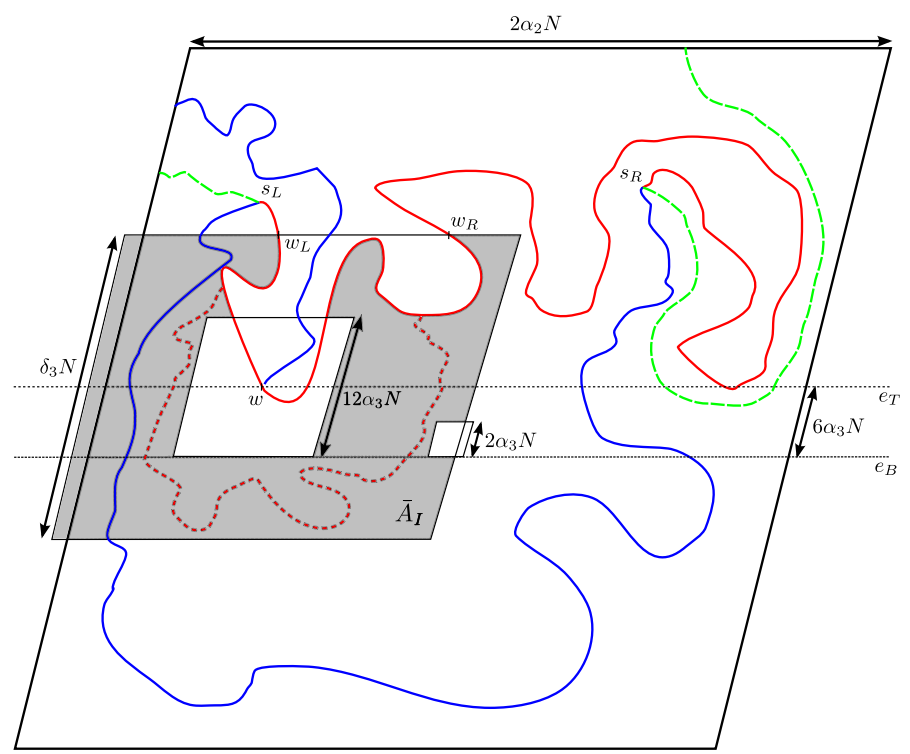

Fig. 7 The grey area represents $\bar{A}_{I}$. If there is no open arm in $\bar{A}_{I}$ then there is a closed arc in $\bar{A}_{I}$. This contradicts with $x$ being one of the lowest vertices of $\mathcal{C}_{a}(\lambda)$

For $R \subset \mathbb{T}$ and $r \subset \partial R$ let $\mathcal{T} \mathcal{A}(R, r)$ denote the set of closed vertices $v \in R$ such that $v$ has two non-touching closed arms in $R$ to $r$. Let $M$ denote the connected component of $S_{\text {mid }}(R)$ in $R \backslash e_{T}$. We show the following:

Claim 5.17 Let

$$
E_{5}:=\mathcal{N} \mathcal{A}\left(6 \alpha_{3}, \beta_{4}\right) \cup \mathcal{N} \mathcal{A}\left(\beta_{4}, \delta_{3} / 2\right)
$$

On the event $\bigcap_{i=0}^{5} E_{i} \forall u \in B A^{2}$, the pair $\left(\tilde{\mathcal{R}}-\left\lfloor\alpha_{3} N\right\rfloor z, \tilde{r}_{c}-\left\lfloor\alpha_{3} N\right\rfloor z\right)$ is $\left(\alpha_{3} N, \alpha_{2} N\right)$-regular, and

$$
\mathcal{T} \mathcal{A}\left(\mathcal{R}, r_{c}\right) \cap M=\mathcal{T} \mathcal{A}\left(\tilde{\mathcal{R}}, \tilde{r}_{c}\right) \cap M
$$

In particular,

$$
\mathcal{L}\left(\mathcal{R}, r_{c}\right) \cap B=\mathcal{L}\left(\tilde{\mathcal{R}}, \tilde{r}_{c}\right) \cap B
$$

Proof of Claim 5.17 From the definition of $\left(\tilde{\mathcal{R}}, \tilde{r}_{c}\right)$ it follows that $\left(\mathcal{T} \mathcal{A}\left(\mathcal{R}, r_{c}\right) \cap M\right)$ $\subset\left(\mathcal{T} \mathcal{A}\left(\tilde{\mathcal{R}}, \tilde{r}_{c}\right) \cap M\right)$. Hence it is enough to show that $\left(\mathcal{T} \mathcal{A}\left(\tilde{\mathcal{R}}, \tilde{r}_{c}\right) \backslash \mathcal{T} \mathcal{A}\left(\mathcal{R}, r_{c}\right)\right) \cap$ $M=\emptyset$. Suppose the contrary, that is $\exists v \in\left(\mathcal{T} \mathcal{A}\left(\tilde{\mathcal{R}}, \tilde{r}_{c}\right) \backslash \mathcal{T} \mathcal{A}\left(\mathcal{R}, r_{c}\right)\right) \cap M$. Let $c_{v}^{1}$ and $c_{v}^{2}$ denote two non-touching closed arms starting from $v$ and ending at $v^{1} \in \tilde{r}_{c}$ and $v^{2} \in \tilde{r}_{c}$ respectively. Since $v \in \mathcal{T} \mathcal{A}\left(\mathcal{R}, r_{c}\right) \backslash \mathcal{T} \mathcal{A}\left(\tilde{\mathcal{R}}, \tilde{r}_{c}\right)$, we can assume that $c_{v}^{1}$ cannot be extended in such a way that it connects to $r_{c}$ and this extension is disjoint 


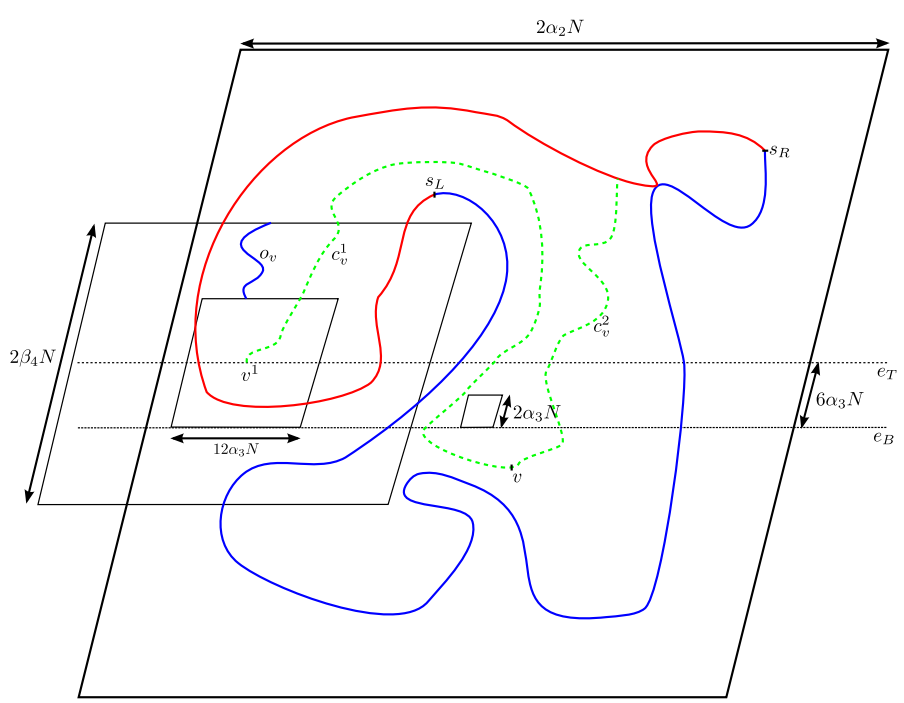

Fig. 8 If $c_{v}^{2} \cap A_{4,0}=\emptyset$, we see 4 half plane arms in $A_{4,0}:$ the two closed induced by $a_{c}$, a closed arm $c_{v}^{1}$, and an open arm $o_{v}$ which separates $c_{v}^{1}$ from $a_{c}$

from and does not touch $c_{v}^{2}$. Hence $v^{1} \in \tilde{r}_{c} \backslash r_{c}$, and $v^{1} \in e_{T}$. Let $S \in \mathcal{W}$ such that $v^{1} \in \partial S$. Note that $\partial S \cap r_{c} \neq \varnothing$. Let $s^{1}, s^{2}$ denote the endpoints of the connected component of $v^{1}$ in $\partial S \cap e_{T}$. At least one of $s^{1}$ and $s^{2}$ is in $r_{c}$. Let $s^{1} \in r_{c}$. Let $\beta_{4} \in$ $\left(6 \alpha_{3}, \delta_{3} / 2\right)$ be an intermediate scale. We divide the annulus $A\left(v^{1} ; 6 \alpha_{3} N, \delta_{3} N / 2\right)$ into the annuli

$$
\begin{aligned}
& A_{4,0}=A\left(v^{1} ; 6 \alpha_{3} N, \beta_{4} N\right), \\
& A_{4,1}=A\left(v^{1} ; \beta_{4} N, \delta_{3} N / 2\right) .
\end{aligned}
$$

We have two cases. If $c_{v}^{2} \cap A_{4,0} \neq \varnothing$, then we see 4 half plane arms in $A_{4,1}: a_{c}$ provides two closed arms, and each of $c_{v}^{1}$ and $c_{v}^{2}$ gives one closed arm. Hence the event $\mathcal{N} \mathcal{A}^{c}\left(6 \alpha_{3}, \beta_{4}\right)$ occurs. If $c_{v}^{2} \cap A_{4,0}=\emptyset$, we have 4 half plane arms in $A_{4,0}: a_{c}$ provides two closed arms, $c_{v}^{1}$ another closed arm, moreover, we get an open arm which separates $c_{v}^{1}$ from $a_{c}$. See Fig. 8 for more details. Hence the event $\mathcal{N} \mathcal{A}^{c}\left(\beta_{4}, \delta_{3} / 2\right)$ occurs. By (5.23) this finishes the proof of Claim 5.17.

By Corollary 2.13 we set $\alpha_{3}$ such that

$$
\mathbb{P}\left(E_{4} \cap E_{5}\right) \geq 1-\varepsilon / 20
$$

for $N \geq N_{5}\left(\varepsilon, \alpha_{3}, \lambda_{0}, \lambda, \alpha, K\right)$. Let

$$
E=E_{0} \cap E_{1} \cap E_{2} \cap E_{3} \cap E_{4} \cap E_{5} .
$$


The combination of the lines in the beginning of Step 1, (5.18), (5.21), (5.22) and (5.24) gives that

$$
\mathbb{P}(E) \geq 1-\varepsilon / 4
$$

for $N \geq \bigvee_{i=0}^{5} N_{i}$. This finishes Step 6 .

Step 7. We set $\theta>0$ such that $\mathbb{P}_{N}\left(B A^{2} \neq \emptyset\right)<\varepsilon / 2$ for large $N$, and conclude the proof of Proposition 3.5.

For $v \in V$, let

$$
Z(v):=\left\{\exists u \in B A^{2} \text { such that } z(u)=v\right\}
$$

Hence

$$
\left\{B A^{2} \neq \varnothing\right\}=\underset{v \in B\left(\left\lceil\frac{2 \alpha+K+2}{\alpha_{3}}\right\rceil\right)}{\bigcup} Z(v)
$$

and

$$
\mathbb{P}_{N}\left(B A^{2} \neq \emptyset, E\right) \leq \sum_{v \in B\left(\left\lceil\frac{2 \alpha+K+2}{\alpha_{3}}\right\rceil\right)} \mathbb{P}_{N}(Z(v) \cap E)
$$

Note that on the event $Z(v) \cap E$, Claim 5.10 and the arguments above give that $\mathcal{C}_{a}(u ; \lambda), F(u), \lambda_{F}(u), \mathcal{R}(u), r_{c}(u), \tilde{\mathcal{R}}(u)$ and $\tilde{r}_{c}(u)$ do not depend on the choice of $u \in B A^{2}$ as long as $z(u)=v$. Except for $\mathcal{C}_{a}(u, \lambda)$, we omit the argument $u$ from the notation above.

We set $k:=\lfloor 1 / 2 \theta\rfloor$. Recall that $d(x, y)=d(\tilde{x}, \tilde{y})+\sqrt{3}=\operatorname{diam}\left(\mathcal{C}_{a}(u ; \lambda)\right)+\sqrt{3}$, and $\operatorname{diam}\left(\mathcal{C}_{a}(u ; \lambda)\right) \in((\alpha-\theta) N,(\alpha+\theta) N)$. On the event $Z(v)$ there is a unique $l=l(y) \in[0, k-1] \cap \mathbb{Z}$ such that $x \in B_{l, k}$ where

$$
\begin{aligned}
B_{l, k} & =B_{l, k}(v) \\
& :=\left[-\alpha_{3} N, \alpha_{3} N\right] \otimes\left(\left(2 \frac{l}{k}-1\right) \alpha_{3} N,\left(2 \frac{l+1}{k}-1\right) \alpha_{3} N\right]+\left\lfloor\alpha_{3} N\right\rfloor v .
\end{aligned}
$$

Recall from the lines above Step 5 we have $x \in \mathcal{L}\left(\mathcal{R}, r_{c}\right)$. From Claim 5.17 we have $\mathcal{L}\left(\mathcal{R}, r_{c}\right) \cap B=\mathcal{L}\left(\tilde{\mathcal{R}}, \tilde{r}_{c}\right) \cap B$ where $B=B\left(\left\lfloor\alpha_{3} N\right\rfloor v ; \alpha_{3} N\right)$. Hence on the event $Z(v) \cap E$, we have $\mathcal{L}\left(\tilde{\mathcal{R}}, \tilde{r}_{c}\right) \cap B_{l, k} \neq \emptyset$. Let $(R, r)$ be a fixed pair. Hence

$$
\begin{aligned}
& \mathbb{P}_{N}\left(Z(v), E,\left(\mathcal{R}, r_{c}\right)=(R, r)\right) \\
& =\mathbb{P}_{N}\left(Z(v), E,\left(\mathcal{R}, r_{c}\right)=(R, r), \mathcal{L}(\tilde{R}, \tilde{r}) \cap B_{l, k} \neq \emptyset \text { at time } p_{\lambda_{F}}(N)\right)
\end{aligned}
$$

where $(\tilde{R}, \tilde{r})$ denotes the pair we get when we cut down some parts of $R$ as in Step 6 .

Recall Definition 5.8. Lemma 5.9 gives that the $N$-parameter frozen percolation process is adapted to the filtration $\left(\mathcal{F}_{t}(V)\right)_{t \in[0,1]}$. Hence for all $u \in B A^{2}, l$ and $\lambda_{F}$ are $\mathcal{F}_{p_{\lambda}(N)}(V)$-measurable functions, and $\left\{\left(\mathcal{R}, r_{c}\right)=(R, r)\right\} \in \mathcal{F}_{p_{\lambda}(N)}(V)$. By 
Claim 5.12 we have that on the event $Z(v) \cap E \cap\left\{\left(\mathcal{R}, r_{c}\right)=(R, r)\right\}$ the $\tau$-values in $R$ do not influence the frozen percolation process in $V \backslash R$ up to time $p_{\lambda}(N)$. This combined with Claim 5.15 gives that there is a function $f$ such that $f\left(R, \bar{l}, \bar{\lambda}_{F}\right)$ is $\mathcal{F}_{p_{\lambda}(N)}(V \backslash R)$-measurable for all $R, \bar{l}, \bar{\lambda}_{F}$. Moreover, it satisfies

$$
\mathbf{1}\left\{Z(v), E,\left(\mathcal{R}, r_{c}\right)=(R, r), l=\bar{l}, \lambda_{F} \in d \bar{\lambda}_{F}\right\}=f\left(R, \bar{l}, \bar{\lambda}_{F}\right) \mathbf{1}\{Z(v), E\}
$$

for $\bar{l} \in[0, k-1] \cap \mathbb{Z}$ and Lebesgue almost every $\bar{\lambda}_{F} \in[0,1]$.

Hence

$$
\begin{aligned}
& \mathbb{P}_{N}\left(\begin{array}{c}
Z(v), E, l=\bar{l}, \lambda_{F} \in d \bar{\lambda}_{F} \\
\mathcal{L}(\tilde{R}, \tilde{r}) \cap B_{\bar{l}, k} \neq \emptyset \text { at time } p_{\bar{\lambda}_{F}}(N) \mid \mathcal{F}_{p_{\lambda}(N)}(V \backslash R)
\end{array}\right) \\
& =f\left(R, \bar{l}, \bar{\lambda}_{F}\right) \mathbb{P}_{N}\left(\begin{array}{c}
Z(v), E \\
\mathcal{L}(\tilde{R}, \tilde{r}) \cap B_{\bar{l}, k} \neq \emptyset \text { at time } p_{\bar{\lambda}_{F}}(N)
\end{array} \mid \mathcal{F}_{p_{\lambda}(N)}(V \backslash R)\right)
\end{aligned}
$$

for $\bar{l} \in[0, k-1] \cap \mathbb{Z}$ and Lebesgue almost every $\bar{\lambda}_{F} \in[0,1]$.

From Step 6, we have that $\tilde{R} \subseteq R$. Claim 5.17 shows that we can apply Corollary 5.7 in the following. We have

$$
\begin{aligned}
\mathbb{P}_{N}\left(Z(v), E, \mathcal{L}(\tilde{R}, \tilde{r}) \cap B_{\overline{l, k}} \neq \emptyset \text { at time } p_{\bar{\lambda}_{F}}(N) \mid \mathcal{F}_{p_{\lambda}(N)}(V \backslash R)\right) \\
\quad \leq \mathbb{P}_{N}\left(\mathcal{L}(\tilde{R}, \tilde{r}) \cap B_{\bar{l}, k} \neq \emptyset \text { at time } p_{\bar{\lambda}_{F}}(N) \mid \mathcal{F}_{p_{\lambda}(N)}(V \backslash R)\right) \\
\quad=\mathbb{P}_{p_{\bar{\lambda}_{F}}(N)}\left(\mathcal{L}(\tilde{R}, \tilde{r}) \cap B_{\bar{l}_{, k}} \neq \emptyset\right) \\
\quad \leq c_{1} k^{-1}
\end{aligned}
$$

for $N \geq N_{6}\left(\lambda_{0}, \lambda, \alpha_{3}, \alpha_{2}, k\right)$ with $c_{1}=c_{1}\left(\lambda_{0}, \lambda, \alpha_{3}, \alpha_{2}\right)$ of Corollary 5.7. A combination of (5.29) and (5.28) gives that

$$
\begin{gathered}
\mathbb{P}_{N}\left(\begin{array}{c}
Z(v), E,\left(\mathcal{R}, r_{c}\right)=(R, r), l=\bar{l}, \lambda_{F}=\bar{\lambda}_{F}, \\
\mathcal{L}(\tilde{R}, \tilde{r}) \cap B_{l, k} \neq \emptyset \text { at time } p_{\lambda_{F}}(N) \mid \mathcal{F}_{p_{\lambda}(N)}(V \backslash R)
\end{array}\right) \\
\leq c_{1} k^{-1} f\left(R, \bar{l}, \bar{\lambda}_{F}\right)
\end{gathered}
$$

for $N \geq N_{6}$. Hence

$$
\mathbb{P}_{N}(Z(v) \cap E) \leq c_{1} k^{-1}
$$

for $N \geq N_{6}$. 
(5.30) combined with (5.26) gives that

$$
\begin{aligned}
\mathbb{P}_{N}\left(B A^{2} \neq \emptyset, E\right) & \leq \sum_{v \in B\left(\left\lceil\frac{2 \alpha+K+2}{\alpha_{3}}\right\rceil\right)} \mathbb{P}_{N}(Z(v) \cap E) \\
& \leq c_{2} k^{-1}
\end{aligned}
$$

with $c_{2}=c_{2}\left(\lambda_{0}, \lambda, \alpha_{3}, \alpha_{2}, K\right)$ for $N \geq N_{6}$. We set $\theta$ such that $k=\lfloor 1 / 2 \theta\rfloor>4 c_{2} / \varepsilon$. A combination of (5.31) and (5.25) gives that

$$
\begin{aligned}
\mathbb{P}_{N}\left(B A^{2} \neq \emptyset\right) & \leq \mathbb{P}_{N}\left(B A^{2} \neq \emptyset, E\right)+\mathbb{P}_{N}\left(E^{c}\right) \\
& \leq c_{2} k^{-1}+\varepsilon / 4<\varepsilon / 2
\end{aligned}
$$

for $N \geq N^{\prime}=\bigvee_{i=0}^{6} N_{i}$.

A proof analogous to that of (5.32) gives that there is $N^{\prime \prime}=N^{\prime \prime}(\alpha, \lambda, K)$

$$
\mathbb{P}_{N}\left(B A^{1} \neq \varnothing\right)<\varepsilon / 2
$$

for $N \geq N^{\prime \prime}$. A combination of (5.17), (5.32) and (5.33) finishes the proof of Proposition 3.5 .

Acknowledgments The author is grateful for the hospitality of Centrum Wiskunde \& Informatica (CWI), Amsterdam, Netherlands where he was employed while the research in this article has been carried out. His research was funded by the Netherlands Organization for Scientific Research (NWO) during that period. The author thanks Jacob van den Berg, Federico Camia, Pierre Nolin, Gábor Pete and Vincent Tassion for fruitful discussions. He thanks Gábor Pete and Artëm Sapozhnikov for calling his attention to Lemma 2.11, which was instrumental in the proof of the main result. He is grateful to Jacob van den Berg, René Conijn and Artëm Sapozhnikov for their numerous comments on the earlier versions of the paper. In particular, the author thanks Artëm Sapozhnikov for his remarks which led to a simpler proof of Lemma 5.3.

\section{Appendix}

\subsection{Winding number of arms}

Here we prove Proposition 2.7. The proof is motivated by [5]. There, among many other things, it was shown that when there are $k$ disjoint open arms in $A(M, a M)$ $(a>1)$, then, with conditional probability at least $1-a^{-\varepsilon}$, and uniformly in $M$, are also $k$ disjoint open arms which wind around the origin at least $c \log a$ times where $c, \varepsilon$ are positive constants. The arguments here are similar to those in Step 2 of the proof of Theorem 5 in [5].

We prove a slightly different result, namely that if we have $k$ disjoint arms with any colour sequence $\sigma \in\{o, c\}^{k}$ in $A(M, a M)$, than with conditional probability at least $1-a^{-\varepsilon}$, these arms wind around the origin at in at least $c \log a$ disjoint subannuli of $A(a, b)$ for some $c, \varepsilon>0$. Following [19], we recall the notion of well separated arms. We modify Definition 7 of [19] for annuli: 
Definition 6.1 Consider some annulus $A=A(v ; M, \tau M)$ and a parallelogram $B=$ $B(v ; \tau M)$ for $M \in \mathbb{N}, \tau \in(1, \infty)$ and $v \in V$. Let $s_{T}, s_{B}, s_{L}, s_{R}$ denote the top, bottom, left and right sides of $B$. Let $\mathcal{C}=\left\{c_{i}\right\}_{1 \leq i \leq j}$ be a set of $j$ disjoint arms in $A$ such that for each $i$, all of the vertices of $c_{i}$ are open or all of them are closed. Let $z_{i}$ be the endpoint of $c_{i}$ on $\partial B(v ; \tau M)$. Let $\eta \in(0,1]$, we attach a parallelogram $r_{i}$ to $z_{i}$ as follows:

$$
r_{i}= \begin{cases}z_{i}+[-\eta M, \eta M] \otimes[0,2 \sqrt{\eta} M] & \text { if } z_{i} \in s_{T} \\ z_{i}+[-\eta M, \eta M] \otimes[0,-2 \sqrt{\eta} M] & \text { if } z_{i} \in s_{B} \\ z_{i}+[-2 \sqrt{\eta} M, 0] \otimes[-\eta M, \eta M] & \text { if } z_{i} \in s_{L} \\ z_{i}+[0,2 \sqrt{\eta} M] \otimes[-\eta M, \eta M] & \text { if } z_{i} \in s_{R} .\end{cases}
$$

We say that $\mathcal{C}$ is $\eta$-well-separated on the outside, if the two following conditions are satisfied:

1. The extremities $z_{i} i=1,2, \ldots, j$ are neither too close to each other:

$$
\forall i \neq l, d\left(z_{i}, z_{l}\right) \geq 10 \sqrt{\eta} M
$$

nor too close to the corners $Z_{l} l=1,2,3,4$ of $B$ :

$$
\forall i, j, d\left(z_{i}, Z_{l}\right) \geq 10 \sqrt{\eta} M
$$

2. Each $r_{i}$ is crossed vertically when $z_{i} \in s_{T} \cup s_{B}$, and horizontally when $z_{i} \in s_{L} \cup s_{R}$ by some crossing $\tilde{c}_{i}$ of the same colour as $c_{i}$, and

$$
c_{i} \text { is connected to } \tilde{c}_{i} \text { in } z_{i}+A(1, \sqrt{\eta} M) .
$$

We say that a set $\mathcal{C}=\left\{c_{i}\right\}_{1 \leq i \leq j}$ of disjoint arms in $A$ can be made $\eta$-well-separated on the outside, if there exists an set $\mathcal{C}^{\prime}=\left\{c_{i}^{\prime}\right\}_{1 \leq i \leq j}$ of disjoint arms in $A$ which is $\eta$-well-separated on the outside, and $c_{i}^{\prime}$ has the same colour and endpoint on $\partial B(v ; M)$ as $c_{i}$ for $i=1,2, \ldots, j$.

Similarly to Definition 6.1, we define the $\eta$-well-separation on the inside. The following statement follows from Lemma 15 of [19].

Lemma 6.2 For $\tau \in(1, \infty)$, and $\delta>0$, there exists $\eta(\delta)>0$ such that for any positive integer $N$, we have

$$
\begin{gathered}
\mathbb{P}_{1 / 2} \text { (any set of disjoint arms in } A(N, \tau N) \text { can be made } \\
\eta \text {-well-separated on the outside }) \geq 1-\delta .
\end{gathered}
$$

Moreover, the same statement holds for well separated arms on the inside.

We prove the following proposition.

Proposition 6.3 Let $k, N \in \mathbb{N}, a \in(10, \infty)$, and $\sigma$ a colour sequence of length $k$. We divide the annulus $A(N, a N)$ into the annuli $A_{i}=A\left(2^{i} N, 2^{i+1} N\right)$ for $i=$ $0,1, \ldots,\left\lfloor\log _{2}(a)\right\rfloor-1$. Let $W$ denote the set of indices $i$ for which all the arms 
in $A_{3 i+1}$ wind around the origin at least once in the counter-clockwise direction for $i=0,1, \ldots,\left\lfloor\log _{2}(a) / 3\right\rfloor-1$. There are positive constants $c=c(k), \varepsilon=\varepsilon(k)$ and $N_{0}=N_{0}(k)$ such that

$$
\mathbb{P}_{1 / 2}\left(\mathcal{A}_{k, \sigma}(N, a N),|W| \geq c \log _{2} a\right) \geq\left(1-a^{-\varepsilon}\right) \pi_{k, \sigma}(N, a N)
$$

for all $a \in(1, \infty)$ and $N \geq N_{0}$.

Remark 6.4 Proposition 2.7 follows from Proposition 6.3, since $W=\emptyset$ on the event $\mathcal{A}_{k, l, \sigma}(N, a N)$ when $l \geq 1$.

Proof of Proposition 6.3 For $a \leq 2$, the statement is trivial. Hence in the rest of the proof we suppose that $a>2$. Classical RSW techniques [15] give that for all $k \in \mathbb{N}$ there is $\varepsilon_{1}=\varepsilon_{1}(k)>0$ such that

$$
\pi_{k, \sigma}(N, a N) \geq a^{-\varepsilon_{1}}
$$

uniformly in $a \geq 2, N \geq 1$ and $\sigma \in\{o, c\}^{k}$.

Let $\eta \in(0,1 / 10)$. Let $I S_{i}\left(O S_{i}\right)$ denote the event that any set of disjoint arms of $A_{i}$ can be made $\eta$-well-separated on the inside (outside). Let $W S$ denote the set of indices $i \in\left\{0,1, \ldots,\left\lfloor\frac{\log _{2} a}{3}\right\rfloor-1\right\}$ for which $O S_{3 i}$ and $I S_{3 i+2}$ both hold. Notice that the events $\{i \in W S\}$ for $i=1,2, \ldots,\left\lfloor\frac{\log _{2} a}{3}\right\rfloor-1$ are independent. Moreover, by Lemma 6.2 , for any $\delta>0$ there is $\eta(\delta) \in(0,1 / 10)$ such that

$$
\mathbb{P}_{1 / 2}(i \in W S) \geq 1-\delta \text {. }
$$

Combining this with Hoeffding's inequality we set $c_{0}, \delta, \eta$ such that

$$
\mathbb{P}_{1 / 2}\left(|W S| \leq c_{0} \log a\right) \leq a^{-2 \varepsilon_{1}}
$$

This and (6.1) gives that

$$
\begin{aligned}
\mathbb{P}_{1 / 2}\left(\mathcal{A}_{k, \sigma}(N, a N) \cap\left\{|W S|>c_{0} \log (a)\right\}\right) & \geq \pi_{k, \sigma}(N, a N)-\mathbb{P}_{1 / 2}\left(|W S| \leq c_{0} \log a\right) \\
& \geq \pi_{k, \sigma}(N, a N)-a^{-2 \varepsilon_{1}} \\
& \geq\left(1-a^{-\varepsilon_{1}}\right) \pi_{k, \sigma}(N, a N)
\end{aligned}
$$

for all $N$.

Let us fix an integer $i \in\left\{0,1, \ldots,\left\lfloor\frac{\log _{2} a}{3}\right\rfloor-1\right\}$. Condition on the event $\mathcal{A}_{k, \sigma}\left(N, 2^{3 i+1} N\right) \cap \mathcal{A}_{k, \sigma}\left(2^{3 i+2} N, a N\right) \cap\{i \in W S\}$ and on the configuration in $A(N, a N) \backslash A_{3 i+1}$. This conditioning gives that all the arms in $A_{3 i}$ can be made $\eta$ well-separated on the outside, and all the arms in $A_{3 i+2}$ can be made $\eta$-well-separated on the inside. This imposes some conditions on the configuration in $A_{3 i+1}$ : there is a finite collection of disjoint parallelograms in which certain crossing events have to be satisfied. In order to have $k$ arms with colour sequence $\sigma$ in $A(N, a N)$, it is enough to connect, with the right colour, the $k$-tuple of parallelograms corresponding to the well separated versions of these arms on the inner parallelogram to those on the outer 
parallelogram of $A_{3 i+1}$. There might be more than one choice for this pair of $k$-tuples of parallelograms. In this case we choose a pair in some deterministic way.

We connect the corresponding pairs of parallelograms by disjoint tubes of width $\sqrt{\eta} 2^{3 i+1} N$ in $A_{3 i+1}$ as in the proof of Lemma 4 of [16] (see Fig. 9 of [16]), with the difference that these connections are special: We chose these tubes such that each of them winds around the origin at least twice in the counter-clockwise direction. We add an additional tube which avoids the ones above, connects the boundaries of the inner and the outer parallelograms of $A_{3 i+1}$ and winds around the origin at least twice in the counter-clockwise direction.

With standard RSW techniques one can show that the probability of the event that the original tubes are crossed in the hard direction by a path with the appropriate colour, and the additional tube is crossed in the hard direction by an open and a closed path is at least $h>0$. Here $h=h(k, \eta)$ is independent of $i, N$ and the location of the parallelograms we connected. The open and closed crossings of the additional tube forces all the arms of $A(N, a N)$ to wind around the origin in $A_{3 i+1}$ at least once in the counter-clockwise direction. Hence the event $\{i \in W\}$ occurs.

Thus the probability of $\{i \in W\}$ conditioned on the event $\mathcal{A}_{k, \sigma} \cap\{i \in W S\}$ and on the configuration in $A(N, a N) \backslash A_{3 i+1}$ is at least $h$. Note that the event $\{i \in W\}$ only depends on the configuration in $A_{3 i+1}$. Hence, when we condition on the event $\mathcal{A}_{k, \sigma}(N, a N)$ and on the realization of $W S$, the set $W$ stochastically dominates a set $Z$, where the elements of $Z$ are sampled from $W S$ independently from each other with probability $h$.

Hence for $c>0$ we have

$$
\begin{aligned}
& \mathbb{P}_{1 / 2}\left(|W| \geq c \log _{2} a \mid \mathcal{A}_{k, \sigma}(N, a N)\right) \\
& \geq \mathbb{P}_{1 / 2}\left(|W| \geq c \log _{2} a,|W S| \geq c_{0} \log _{2} a \mid \mathcal{A}_{k, \sigma}(N, a N)\right) \\
& =\sum_{S} \mathbb{P}_{1 / 2}\left(|W| \geq c \log _{2} a \mid \mathcal{A}_{k, \sigma}(N, a N), W S=S\right) \mathbb{P}_{1 / 2}\left(W S=S \mid \mathcal{A}_{k, \sigma}(N, a N)\right) \\
& \geq \sum_{S} \mathbb{P}_{1 / 2}\left(|Z| \geq c \log _{2} a \mid \mathcal{A}_{k, \sigma}(N, a N), W S=S\right) \mathbb{P}_{1 / 2}\left(W S=S \mid \mathcal{A}_{k, \sigma}(N, a N)\right),
\end{aligned}
$$

where the summation over $S \subseteq\left\{0,1, \ldots\left\lfloor\frac{\log _{2} a}{3}\right\rfloor-1\right\}$ with $|S| \geq c_{0} \log _{2} a$. We split this sum in (6.4) depending on the number of elements of $S$, and we get

$$
\begin{aligned}
\mathbb{P}_{1 / 2}(|W| & \left.\geq c \log _{2} a \mid \mathcal{A}_{k, \sigma}(N, a N)\right) \\
& \geq \mathbb{P}\left(Y \geq c \log _{2} a\right) \sum_{l \geq c_{0} \log _{2} a} \mathbb{P}_{1 / 2}\left(|W S|=l \mid \mathcal{A}_{k, \sigma}(N, a N)\right) \\
& =\mathbb{P}\left(Y \geq c \log _{2} a\right) \mathbb{P}_{1 / 2}\left(|W S| \geq c_{0} \log _{2} a \mid \mathcal{A}_{k, \sigma}(N, a N)\right),
\end{aligned}
$$

where $Y$ is a random variable with distribution Binom $\left(c_{0} \log _{2} a, h\right)$. Using Hoeffding's inequality, we set $c=c(h), \varepsilon_{2}(h)>0$ such that

$$
\mathbb{P}\left(Y \geq c \log _{2} a\right) \geq 1-a^{-\varepsilon_{2}} .
$$


By substituting (6.6) and (6.3) to (6.5) we get that

$$
\mathbb{P}_{1 / 2}\left(|W| \geq c \log _{2} a \mid \mathcal{A}_{k, \sigma}(N, a N)\right) \geq\left(1-a^{-\varepsilon_{1}}\right)\left(1-a^{-\varepsilon_{2}}\right)
$$

for all $a>2$ and $N$, which finishes the proof of Proposition 6.3.

With suitable adjustments of arguments above, one can show that the following generalization of Proposition 2.7 holds.

Proposition 6.5 For any $k \in \mathbb{N}$, there are positive constants $c=c(k), \varepsilon=\varepsilon(k)$ such that for all $l, l^{\prime} \in \mathbb{N}$ with $0 \leq l<l^{\prime} \leq k$

$$
\pi_{k, l, \sigma}\left(n_{0}(k), N\right) \leq c N^{-\varepsilon} \pi_{k, l^{\prime}, \sigma}\left(n_{0}(k), N\right)
$$

uniformly in $N$ and in the colour sequence $\sigma$.

\subsection{Existence of long thick paths in nice regions}

Recall the Definition 4.3 and 4.4. First we prove Lemma 4.5 which is the special case of Lemma 4.6 where $C$ is $(a, b)$-nice. Then we show how to modify the proof of Lemma 4.5 to deduce Lemma 4.6.

Lemma 4.5 Let $a, b \in \mathbb{N}$ with $a \geq 2000$. Let $C$ be an $(a, b)$-nice subgraph of $\mathbb{T}$. Then there is a $\lfloor a / 200-10\rfloor$-gridpath contained in $C$ with diameter at least $\operatorname{diam}(C)-2 b-2 a-12$.

Remark 6.6 We believe that the constants in Lemma 4.5 are not optimal.

Proof of Lemma 4.5 Recall the lines below Definition 4.4. To prove Lemma 4.5, it is enough to find a path $\zeta$ in $C$ such that $\operatorname{diam}(\zeta) \geq d-2 b-2 a-12$ and $\zeta+$ $B(a / 100-5) \subset C$. We construct $\zeta$ by the following strategy.

We put hexagons on the vertices of ' $T$ in the 'usual' way: The hexagon corresponding to the vertex $v$ is the regular hexagon with side length $1 / \sqrt{3}$ centred around $v$ with one of its sides is vertical. These hexagons give a tiling of the plane $\mathbb{R}^{2}$. Using this tiling, we look at $C$ as the region in $\mathbb{R}^{2}$ which is the union of the hexagons which are centred around the vertices of $C$.

Let $x, y \in C$ such that $d(x, y)=\operatorname{diam}(C)$. Let $\gamma \subset \mathbb{R}^{2}$ be a shortest curve connecting $x$ and $y$ in the region $C$, that is, $\gamma$ is a continuous map of $[0,1]$ such that 0 is mapped to $x$ and 1 is mapped to $y$. We get the path $\zeta$ from $\gamma$ as follows. First we cut down two pieces of $\gamma$ one from its beginning and one from its end. We call the resulting path $\gamma^{2}$. Then we walk along $\gamma^{2}$, and if there is a point of $\partial C$ 'close by' on the left (right, resp.) of $\gamma^{2}$, then we make a 'small' detour to the right (left, resp.). We get the path $\zeta$ from $\gamma^{2}$ after these detours. We show that $\zeta$ indeed satisfies the conditions above, and finish the proof of Lemma 4.5.

We gave a strategy which involved continuous curves and regions in the plane $\mathbb{R}^{2}$. We adapt it to the triangular lattice in the following precise proof. 
Let $x=\left(x_{1}, x_{2}\right), y=\left(y_{1}, y_{2}\right) \in C$ such that $d(x, y)=\operatorname{diam}(C)$. We further assume that $x_{1}<y_{1}$ and $d(x, y)=y_{1}-x_{1}$. The other case where $d(x, y)=y_{2}-x_{2}$ can be treated similarly. Let $\tilde{\gamma}$ denote a shortest (having the least number of vertices) path which starts at $x$, ends at $y$, and it is contained in $C$.

Note that there are $\left(\begin{array}{c}2 n \\ n\end{array}\right)$ shortest paths between the vertices 0 and $n \underline{e}_{1}+n \underline{e}_{2}$ in $\mathbb{T}$. However, most of them do not follow closely the straight line between the points 0 and $n \underline{e}_{1}+n \underline{e}_{2}$. Hence $\tilde{\gamma}$ usually does not resemble a shortest continuous curve connecting $x$ and $y$.

Step 1. We choose a specific shortest path between $x$ and $y$.

For $u, v \in \mathbb{T}$, let $s(u, v)$ denote the line segment connecting $u$ and $v$ in $\mathbb{R}^{2}$. This segment naturally induces an oriented path $\sigma(u, v)$ in $\mathbb{T}$ as a sequence of the midpoints of the hexagons which are intersected by $s(u, v)$ as we walk along it from $u$ to $v$. Note that it can happen that the segment $s(u, v)$ contains a side of a hexagon. In this case, we put only one of the neighbouring hexagons to $\sigma(u, v)$. We say that $\sigma(u, v)$ is a triangular grid approximation of the segment $s(u, v)$. Note that $\sigma(u, v)$ is a shortest path between $u$ and $v$ in $\mathbb{T}$.

Recall the notation in Sect. 4.1. Let $v, u, u^{\prime} \in \tilde{\gamma}$ with $v \prec u, u^{\prime}$ and $u \sim u^{\prime}$. Then for all $w \in \sigma(v, u)$ there is $w^{\prime} \in \sigma\left(v, u^{\prime}\right)$ with $w \sim w^{\prime}$. Hence for $v \in \tilde{\gamma}$ there are two cases:

- either $\forall u \in \tilde{\gamma}_{v, y} \backslash\{v\}$ we have $\sigma(v, u) \backslash\{v\} \nsim \partial C$, or

- $\exists w=w(v) \in \tilde{\gamma}_{v, y} \backslash\{v\}$ such that $\forall u \in \tilde{\gamma}_{v, w} \backslash\{v, w\}$ we have $\sigma(v, u) \backslash\{v\} \nsim \partial C$, but $\sigma(v, w) \backslash\{v\} \sim \partial C$.

We perform the following procedure. We start at $x$. If the first case above holds for $v=x$, then we replace $\tilde{\gamma}$ by $\sigma(x, y)$ and finish the procedure. In the second case we replace $\tilde{\gamma}_{x, w(x)}$ by $\sigma(x, w(x))$, and repeat the procedure for $\tilde{\gamma}_{w(x), y}$ starting from $w(x)$. At each step of the procedure, we move at least one vertex further on $\tilde{\gamma}$, hence the procedure terminates in at most $|\tilde{\gamma}|$ steps. Let $\gamma$ denote the path we get at the end. At each step of the procedure, we make modifications such that the new path is in $C$ and its length is the same as the old path's. Hence $\gamma \subset C$ and $|\gamma|=|\tilde{\gamma}|$.

We finish Step 1 by with the following consequences of the construction above: $\gamma$ resembles a shortest curve in $\mathbb{R}^{2}$ : It is a sequence of triangular grid approximations of line segments in $\mathbb{R}^{2}$. Moreover, we have the following claim.

Claim 6.7 As we walk along $\gamma$, we turn to the left (right, resp.) at $v \in \gamma$ if it has a neighbour in $\partial C$ on the left (right, resp.) of $\gamma$. That is, if $u, v, w \in \gamma$ with $u \prec v \prec w$ and $\sigma(u, v), \sigma(v, w) \subset \gamma$, with $\sigma(u, v) \cup \sigma(v, w) \neq \sigma(u, v)$, then $v \sim \partial C \cap$ $T(u, v, w)$, where $T(u, v, w)$ denotes the triangle spanned by the vertices $u, v, w$.

Step 2. We introduce some notation and assign labels to some of the vertices of $\gamma$. Let

$$
S T:=\left\{v=\left(v_{1}, v_{2}\right) \in V \mid x_{1}<v_{1}<y_{1}\right\} .
$$

By possible shortening $\gamma$ and redefining $x$ and $y$, we can assume that $\gamma \subset \operatorname{cl}(S T)$, $\gamma \cap \partial S T=\{x, y\}$ and $d(x, y)=\operatorname{diam}(C)$. 
We set $\alpha:=\lfloor a / 6\rfloor-2>0$, and define

$$
S T^{i}:=\left\{v=\left(v_{1}, v_{2}\right) \in V \mid x_{1}+b+i \alpha<v_{1}<y_{1}-b-i \alpha\right\}
$$

for $i \in\{1,2\}$. Let $x^{i}$ ( $y^{i}$, resp.) denote the last (first, resp.) vertex of $\gamma$ which is in the half plane $\left\{v=\left(v_{1}, v_{2}\right) \in V \mid v_{1} \leq x_{1}+b+i \alpha\right\}\left(\left\{v \mid v_{1} \geq y_{1}-b-i \alpha\right\}\right.$, resp.). Let $\gamma^{i}=\gamma_{x^{i}, y^{i}}$. Note that $S T^{1} \supset S T^{2}$ and $\gamma^{2}$ is a subpath of $\gamma^{1}$.

Let $i \in\{1,2\}$. Since $\gamma^{i}$ is a shortest path, it is non self-touching. This combined with $\gamma^{i} \cap \partial S T^{i}=\left\{x^{i}, y^{i}\right\}$ we get that $\gamma^{i}$, cuts $c l\left(S T^{i}\right)$ into two connected components. Let $S T_{L}^{i}\left(S T_{R}^{i}\right.$, resp.) denote connected component $c l\left(S T^{i}\right) \backslash \gamma^{i}$ which is on the left (right, resp.) had side of $\gamma^{i}$ as we walk along it.

For $v \in \gamma^{2}$, we put a label $l(v) \in\{L, R, N, G\}$ as follows. We denote the set of vertices with label $X \in\{L, R, N, G\}$ by $\gamma_{X}^{2}$. First we define the labels $R$ and $L$ : For $v \in \gamma^{2}$, we set $l(v)=L\left(l(v)=R\right.$, resp.) if $S T^{1}{ }_{L} \cap B(v ; \alpha) \cap \partial C \neq \varnothing$ $\left(S T^{1}{ }_{R} \cap B(v ; \alpha) \cap \partial C \neq \emptyset\right.$, resp.). To show that the labels $L, R$ are well-defined, we have to check that for $v \in \gamma^{2}$ at most one of the sets $S T^{1}{ }_{L} \cap B(v ; \alpha) \cap \partial C$ and $S T^{1}{ }_{R} \cap B(v ; \alpha) \cap \partial C$ is non-empty. Since $2 \alpha<a$, this follows from Condition 3 of Definition 4.4. Let $\beta:=\lfloor\alpha / 3\rfloor$. For $v \in \gamma^{2} \backslash\left(\gamma_{L}^{2} \cup \gamma_{R}^{2}\right)$ we set $l(v)=G$ if $B(v ; \beta) \cap\left(\gamma_{L}^{2} \cup_{R}^{2}\right)=\emptyset$, and $l(v)=N$ otherwise.

Since $4 \alpha+2 \beta<a$, it is a simple exercise to prove the following claim using Condition 3 of Definition 4.4, which finishes Step 2.

Claim 6.8 Let $u \in \gamma_{L}^{1}$ and $v \in \gamma_{R}^{1}$. Then there is $w \in \gamma_{G}^{1}$ which is in between $u$ and $v$.

Step 3. We define the neighbourhoods $\mathcal{F}_{v}$ and $\mathcal{G}_{v}$ for $v \in \gamma^{2}$.

If $l(v) \in\{G, N\}$ then we set $\mathcal{F}_{v}:=B(v ; \alpha)$ and $\mathcal{G}_{v}:=B(v ; \beta)$.

If $l(v) \in\{L, R\}$, let $f^{1}\left(f^{2}\right.$, resp.) as the last vertex when we go backward (forward, resp.) from $v$ along $\gamma$ which is in $B(v ; \alpha)$. If it has label $L$ ( $R$, resp.) then we define $\mathcal{F}_{v}$ as the connected component of $B(v ; \alpha) \backslash \gamma_{f^{1}, f^{2}}$ on the right (left, resp.) hand side of $\gamma_{f^{1}, f^{2}}$. Similarly we define $g^{1}$ and $g^{2}$ in the box $B(v ; \beta)$, and $\mathcal{G}_{v}$.

The combination of $4 \alpha<a$, Claim 6.7 and Condition 3 of Definition 4.4 gives that

$$
\left(\gamma_{f^{1}, g^{1}} \cup \gamma_{g^{2}, f^{2}}\right) \cap B(v ; \beta-1)=\emptyset .
$$

Hence we get

Claim $6.9 \mathcal{F}_{v} \cap B(v ; \beta)=\mathcal{G}_{v}$ for $v \in \gamma^{2}$.

Step 4. We investigate the neighbourhood $\mathcal{G}_{v}$.

Claim $6.10 \mathcal{G}_{v} \cap \partial C=\emptyset$ for $v \in \gamma^{2}$, and $\mathcal{G}_{v} \cap \gamma^{1}=\emptyset$ for $v \in \gamma_{L}^{2} \cup \gamma_{R}^{2}$.

Proof of Claim 6.10 First we show that $\mathcal{G}_{v} \cap \partial C=\emptyset$ with a proof by contradiction. Suppose that $\mathcal{G}_{v} \cap \partial C \neq \varnothing$. The definition of labels give that if $\mathcal{G}_{v} \cap \partial C \neq \varnothing$, then $l(v)=L$ or $R$. We further suppose that $l(v)=L$. The case where $l(v)=R$ can be 


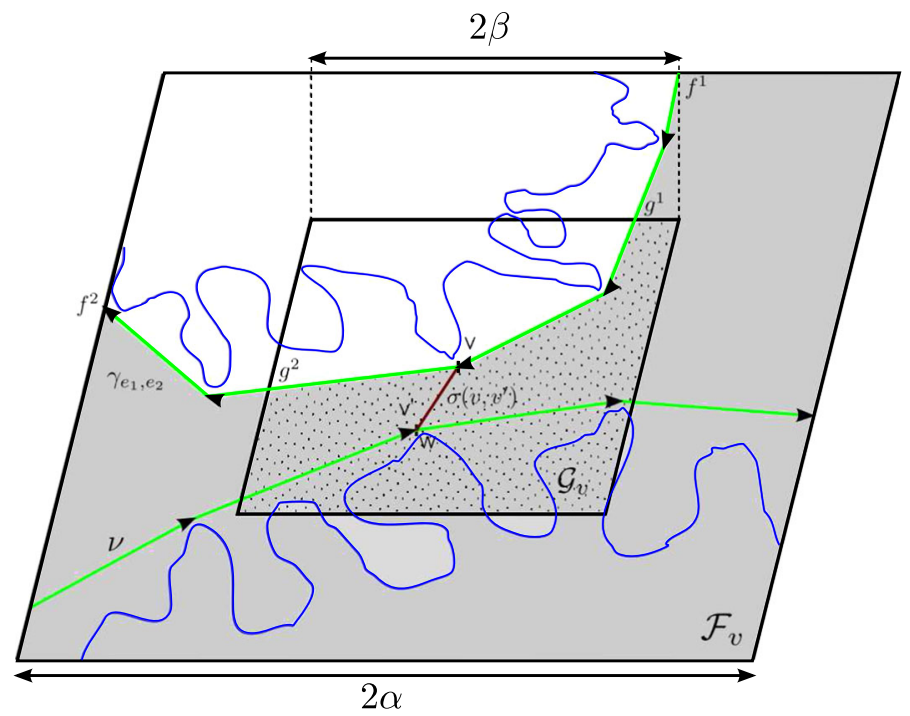

Fig. 9 The path $\gamma_{x, v} \vee \sigma_{v, w} \vee \gamma_{w, y}$, is shorter than $\gamma$ by at least $\frac{2}{3} \alpha$ vertices

treated similarly. We choose $w$ so that it is one of the closest vertices to $v$ among the vertices of $\mathcal{G}_{v} \cap \partial C$. See Fig. 9.

By the definition of the label $L$, we have that $w \in S T_{L}^{2} \cap B(v ; \beta)$. Since $w \in \mathcal{G}_{v}$, i.e. $w$ is on the right hand side of $\gamma_{f^{1}, f^{2}}$ in $B(v ; \alpha)$. Hence some subpath of $\gamma^{1} \backslash \gamma_{f^{1}}, f^{2}$, denoted by $v$, has to separate $w$ from $v$ in $\mathcal{F}_{v}$. Let us walk from $v$ to $w$ on $\sigma(v, w)$, till we hit $v$. Let us denote the explored path by $\sigma\left(v, v^{\prime}\right)$, where $v^{\prime}$ is the last point of the exploration. Let $\gamma^{\prime}$ be the path we get when we replace the part of $\gamma$ between $v$ and $v^{\prime}$ by $\sigma\left(v, v^{\prime}\right)$. Consider the case $v^{\prime} \prec_{\gamma} v$. The other case where $v^{\prime} \succ_{\gamma} v$ can be treated similarly. The number of vertices of $\sigma\left(v, v^{\prime}\right)$ is at most $2 \beta$. However, the number of vertices in $v$ before $v^{\prime}$ is at least $\alpha-\beta$. Moreover, $\left|\gamma_{f^{1}, v}\right| \geq \alpha-\beta$. Hence

$$
\begin{aligned}
|\gamma|-\left|\gamma^{\prime}\right| & \geq 2(\alpha-\beta)-2 \beta \\
& \geq \frac{2}{3} \alpha>0 .
\end{aligned}
$$

The definition of $w$ gives that $\sigma\left(v, v^{\prime}\right) \subset C$, thus $\gamma^{\prime} \subset C$. Hence $\gamma^{\prime}$ connects $x$ and $y$ in $C$ and by 6.7, it is shorter than $\gamma$. This contradicts the definition of $\gamma$, hence $\mathcal{G}_{v} \cap \partial C=\emptyset$ for $v \in \gamma^{2}$.

The proof of $\mathcal{G}_{v} \cap \gamma^{1}=\emptyset$ for $v \in \gamma^{2}$ is quite similar to the one above, hence we omit it, and finish the proof of Claim 6.10 and conclude Step 4.

Step 5. We define the path $\zeta$.

We set $\varepsilon=\lfloor\beta / 4\rfloor-2$. For $j \in\{L, R\}$, let

$$
U_{j}:=\bigcup_{v \in \gamma_{j}^{2}} B(v ; \varepsilon) .
$$


$S T_{R}^{2} \backslash U_{L} \backslash \gamma^{2}\left(S T_{L}^{2} \backslash U_{R} \backslash \gamma^{2}\right.$, resp.) has one infinite connected component which we denote by $Z_{R}\left(Z_{L}\right.$, resp.). Let $\zeta_{j}$ denote the shortest path in $\partial Z_{j} \cap S T^{2}$ which connects the left and the right side of $S T_{2}$. We orient $\zeta_{L}\left(\zeta_{R}\right.$, resp.) so that $Z_{L}\left(Z_{R}\right.$, resp.) is on the left (right, resp.) hand side. Note that $\zeta_{L}, \zeta_{R}$ are left-right crossings of $S T^{2}$.

Note that $\zeta_{L}, \zeta_{R}$ and $\gamma^{2}$ are non self-touching paths. Since $Z_{R}, Z_{L}$ and $\gamma^{2}$ are disjoint, $\gamma^{2}$ is sandwiched between $\zeta_{L}$ and $\zeta_{R}$. Hence $\zeta_{L}, \zeta_{R}, \gamma^{2}$ can have common vertices, but they cannot cross each other. Thus we get the following claim.

Claim 6.11 Let $v \in \zeta_{L} \cap \zeta_{R}$. Then $v \in \gamma^{2}$.

Condition 3 of Definition 4.4 implies the following claim.

Claim 6.12 Let $v \in \zeta_{L} \cap \zeta_{R}$. If $w$, the next vertex after $v$ on $\gamma^{2}$ exists, then $w \in \zeta_{L} \cup \zeta_{R}$.

Let $\vec{G}=(\vec{V}, \vec{E})$ be the directed graph induced by the directed paths $\zeta_{L}, \zeta_{R}$ and $\gamma^{2}$. That is $\vec{G}=(\vec{V}, \vec{E})$ where $\vec{V}=\zeta_{L} \cup \zeta_{R} \cup \gamma^{2}$, and $(u, v) \in \vec{E}$ if and only if $u, v \in v, u \sim v$ and $u \prec_{v} v$ for some $v \in\left\{\zeta_{L}, \zeta_{R}, \gamma^{2}\right\}$. Using the definition of $\zeta_{L}$ and $\zeta_{R}$ it is a simple exercise to show the following claim.

Claim $6.13 \vec{G}$ has no directed loops.

For $j \in\{L, R\}$ and $z \in \zeta_{j}$ let $n_{j}(z)$ be the first vertex of $\zeta_{j} \cap \gamma^{2}$ after $z$ on $\zeta_{j}$. That is, $n_{j}(z) \in \zeta_{j} \cap \gamma^{2}$ with $n_{j}(z) \succeq_{\zeta_{j}} z$ and if $z^{\prime} \in \zeta_{j} \cap \gamma^{2}$ with $z^{\prime} \succ_{\zeta_{j}} z$ then $z^{\prime} \succeq_{\zeta_{j}} n_{j}(z)$. If there is no such vertex, then we set $n_{j}(z)=\emptyset$.

We define a directed path $\zeta$ by the following procedure. Let $z_{j}$ denote the starting point of $\zeta_{j}$ for $j \in\{L, R\}$. $\zeta$ starts at the vertex $z$ defined as

$$
z:= \begin{cases}z_{L} \quad \text { when } n_{L}\left(z_{L}\right)=\emptyset, \text { or, when } n_{L}\left(z_{L}\right) \neq \emptyset \neq n_{R}\left(z_{R}\right), \text { and } \\ & n_{L}\left(z_{L}\right) \succeq_{\gamma^{2}} n_{R}\left(z_{R}\right) \\ z_{R} & \text { otherwise. }\end{cases}
$$

Suppose that we are at vertex $v$ in $\zeta$. If $v$ is the endpoint of $\zeta_{L}$ or $\zeta_{R}$, we terminate the procedure. Otherwise, we define the next vertex of $\zeta$, denoted by $w$, as follows. For $j \in\{L, R\}$, if $v \in \zeta_{j}$, then $v_{j}$ denotes the next vertex after $v$ in $\zeta_{j}$.

- If $v \in \zeta_{L} \backslash \zeta_{R}$, then $w=v_{L}$

- if $v \in \zeta_{R} \backslash \zeta_{L}$, then $w=v_{R}$

- if $v \in \zeta_{L} \cap \zeta_{R}$, and if

- $v_{L}, v_{R} \in \gamma^{2}$, then the definition of $\zeta_{L}$ and $\zeta_{R}$ gives that $v_{L}=v_{R}$ and we take $w=v_{L}=v_{R}$

- $v_{L} \in \gamma^{2}, v_{R} \notin \gamma^{2}$, then $w=v_{R}$

- $v_{R} \in \gamma^{2}, v_{L} \notin \gamma^{2}$, then $w=v_{L}$

- the case $v_{L}, v_{R} \notin \gamma^{2}$ is impossible by Claim 6.12.

We finish Step 5 by showing that $\zeta$ is well-defined. The definition of $\zeta$ shows that if we view $\zeta$ as a directed graph, it is a subgraph of $\vec{G}$. Hence by Claim $6.13 \zeta$ has no directed loops. Thus $\zeta$ is self avoiding, and the procedure above terminates after finitely many steps, when $\zeta$ reaches the endpoint of $\zeta_{L}$ or $\zeta_{R}$.

Step 6. We prove the following claim and finish the proof of Lemma 4.5. 
Claim $6.14 \zeta+B(\varepsilon) \subset C$ and $\operatorname{diam}(\zeta) \geq d(x, y)-2 b-4 \alpha$.

Proof of Claim 6.14 The definition of $\zeta$ shows that $\zeta$ is a horizontal crossing of $S T^{2}$. Hence $\operatorname{diam}(\zeta) \geq d(x, y)-2 b-4 a$. We show that for all $v \in \zeta$ we have $v+B(\varepsilon) \subset C$. There are two cases depending on whether $v$ is contained in $\gamma^{2}$.

Case 1: $v \in \zeta \backslash \gamma^{2}$. Then $v \in \zeta_{L} \backslash \gamma^{2}$ or $v \in \zeta_{R} \backslash \gamma^{2}$. We assume that $v \in \zeta_{L} \backslash \gamma^{2}$. The case where $v \in \zeta_{R} \backslash \gamma^{2}$ can be treated similarly. The definition of $\zeta_{L}$ gives that there is $w \in \gamma_{R}$ such that $v \in(B(w ; \varepsilon+1) \backslash B(w ; \varepsilon))$ and $B(v ; \varepsilon) \cap \gamma_{R}=\emptyset$. This combined with $4 \alpha+4 \varepsilon+2<a$ and Condition 3 of Definition 4.4 gives that $B(v ; \varepsilon) \cap\left(\gamma_{L}^{2} \cup \gamma_{R}^{2}\right)=\emptyset$.

If $\gamma^{2} \cap B(v ; \varepsilon) \neq \emptyset$, then $\exists u \in\left(\gamma_{G}^{2} \cup \gamma_{N}^{2}\right) \cap B(v ; \varepsilon)$. Claim 6.10 implies that $C \supset \mathcal{G}_{u}=B(u ; \beta) \supset B(v ; \varepsilon)$ since $4 \varepsilon<\beta$.

If $\gamma^{2} \cap B(v ; \varepsilon)=\emptyset$, then the definition of $w$ and Claim 6.10 shows that $C \supset \mathcal{G}_{w} \supset$ $B(v ; \varepsilon)$ since $2 \beta+2 \varepsilon<\alpha$.

Hence $B(v ; \varepsilon) \subset C$ in Case 1 .

Case 2: $v \in \zeta \cap \gamma^{2}$. Since $\zeta \subset \zeta_{L} \cup \zeta_{R}$, we assume that $v \in \zeta_{L}$. The case where $v \in \zeta_{R}$ can be treated similarly. First we show that $v \notin \gamma_{L}^{2} \cap \zeta_{L}$.

Suppose the contrary, that is $v \in \gamma_{L}^{2} \cap \zeta_{L}$. Let $w$ be the starting point of the connected component of $v$ in $\gamma^{2} \cap \zeta$. By the definition of $\zeta, w \in \zeta_{L}$. Moreover, for $w^{\prime}$ the vertex right before $w$ on $\zeta_{L}$, we have $w^{\prime} \in \zeta_{L} \backslash \gamma_{2}$. Hence there is $u^{\prime} \in \gamma_{R}^{2}$ such that $w^{\prime} \in B\left(u^{\prime} ; \varepsilon+1\right)$. Since $v \in \gamma_{L}$ and $u^{\prime} \in \gamma_{R}^{2}$, by Claim $6.8 \exists u \in \gamma_{G}^{2}$ which is between $u^{\prime}$ and $v$ on $\gamma^{2}$. Note that $w^{\prime} \in \mathcal{G}_{u^{\prime}}$. By Claim 6.10 we have that $\gamma_{u^{\prime}, w}^{2} \subset \gamma^{2} \backslash \gamma_{G}^{2}$. Hence $u$ is between $w$ and $v$ on $\gamma^{2}$. From the definition of $w$, we get that $u \in \zeta \cap \zeta_{L}$.

Note that if we show that $u \in \zeta_{R}$, then we get a contradiction by the definition of $\zeta$. Hence in order to rule out the case $v \in \gamma_{L}^{2} \cap \zeta_{L}$ it is enough to show that $u \in \zeta_{R}$.

Suppose the contrary, that is $u \notin \zeta_{R}$. Recall the definition of $U_{L}$ from 6.8. We introduce a new set of labels on the vertices of $U_{L}$ as follows. For $q \in U_{L}$ there is a vertex $r \in \gamma_{L}$ such that $q \in B(r ; \varepsilon)$. We define

$$
l^{\prime}(q):= \begin{cases}B & \text { if } r \prec \gamma^{2} u \\ A & \text { otherwise. }\end{cases}
$$

Since the choice of $r$ above is not necessarily unique, we have to show that $l^{\prime}(q)$ is well-defined. It can be easily checked by combining Claim $6.10,4 \varepsilon+4<\beta$ and $u \in \gamma_{G}^{2}$. Moreover a similar argument shows that if $q, q^{\prime} \in U_{L}$ with $q \sim q^{\prime}$, then $l^{\prime}(q)=l^{\prime}\left(q^{\prime}\right)$.

Since $\gamma^{2}$ is non self-touching, $u \in \gamma^{2}$ is connected to $\infty$ in $S T_{R}$. Since $u \notin \zeta_{R}$ it is not connected to $\infty$ in $Z_{R}$, there is a path $v \subset U_{L}$ which separates $u$ from $\infty$ in $S T_{R}$. We can choose $v$ such that it starts and ends at a vertex neighbouring $\gamma^{2}$. By a possible shortening of $v$, we can assume that if $u^{\prime} \in v$ with $u^{\prime} \sim \gamma^{2}$, than $u^{\prime}$ is either the starting or the endpoint of $v$. Let $u_{1}, u_{2}$ be neighbours of the starting point and the endpoint of $v$ which are in $\gamma^{2}$. The definition of $v$ gives that $u$ is in between $u_{1}$ and $u_{2}$ on $\gamma^{2}$. Using Condition 3 of Definition 4.4 and that $u \in \gamma_{G}^{2}$ it is easy to check that $l^{\prime}\left(u_{1}\right) \neq l^{\prime}\left(u_{2}\right)$. 
On the other hand, $v$ is a connected subset of $U_{L}$, hence $l^{\prime}$ is constant on $v$. This is a contradiction, thus $u \in \zeta_{R}$, which in turn shows that $v \in \gamma_{L}^{2} \cap \zeta_{L}$.

Hence $v \notin \gamma_{L}^{2} \cap \zeta_{L}$ but $v \in \zeta \cap \gamma^{2} \cap \zeta_{L}$. The definition of $\zeta_{L}$ gives that $v \notin \gamma_{R}$. Hence $v \in \gamma_{N}^{2} \cup \gamma_{G}^{2}$. By Claim 6.10 we get $C \supset \mathcal{G}_{v}=B(v ; \beta) \supset B(v ; \varepsilon)$, and we are done in Case 2. Since there are no other cases left, the proof of Claim 6.14 is finished.

Since $\zeta+B(\varepsilon) \subset C$ and $\operatorname{diam}(\zeta) \geq d(x, y)-2 b-4 \alpha$ hence the $\lfloor\varepsilon / 2\rfloor$-gridpath approximation of $\zeta$ is contained in $C$. It has diameter at least $d(x, y)-2 b-4 \alpha-\varepsilon \geq$ $d(x, y)-2 b-2 a-12$. Since $\varepsilon=\lfloor\beta / 4\rfloor-2 \geq a / 100-5$ this concludes the proof of the Lemma 4.5 .

We finish the appendix by proving Lemma 4.6.

Lemma 4.6 Let $a, b, c \in \mathbb{N}$ with $a \geq 2000$. Let $C$ be subgraph of $\mathbb{T}$ which is $(a, b)$-nice in $B(c)$. Let $C^{\prime}$ be a connected component of $C \cap B(c)$. Then there is a $\lfloor a / 200-10\rfloor$-gridpath contained in $C^{\prime}$ with diameter at least diam $\left(C^{\prime}\right)-2 b-2 a-12$.

Proof of Lemma 4.6 Let $x, y \in C^{\prime}$ with $d(x, y)=\operatorname{diam}\left(C^{\prime}\right)$. We choose $\tilde{\gamma}$ as one of the shortest paths connecting $x, y$ in $C^{\prime}$. From this point on, we can follow the proof of Lemma 4.5 since we will use Condition 3 of Definition 4.4 for pairs of vertices $u, v \in \partial C$ which are contained in $B(c)$.

\section{References}

1. Ahlberg, D., Duminil-Copin, H., Kozma, G., Sidoravicius, V.: Seven-dimensional forest-fires. Annales de 1'Institut Henri Poincaré Probabilités et Statistiques (2013, appear). arXiv:1302.6872

2. Ahlberg, D., Sidoravicius, V., Tykesson, J.: Bernoulli and self-destructive percolation on non-amenable graphs. Electron. Commun. Probab. 19(40), 1-6 (2014)

3. Aizenmann, M., Barsky, D.J.: Sharpness of the phase transition in percolation models. Commun. Math. Phys. 108(3), 489-526 (1987)

4. Aldous, D.J.: The percolation process on a tree where infinite clusters are frozen. Math. Proc. Camb. Philos. Soc. 128, 465-477 (2000)

5. Beffara, V., Nolin, P.: On monochromatic arm exponents for 2D critical percolation. Ann. Probab. 39, 1286-1304 (2011)

6. Benjamini, I., Schramm, O.: Private communication with David Aldous (1999)

7. Bertoin, J.: Random fragmentation and coagulation processes. In: Cambridge Studies in Advanced Mathematics, vol. 102. Cambridge University Press, Cambridge (2006)

8. Bertoin, J.: Fires on trees. Ann. Instit. H. Poincaré Probab. Stat. 48(4), 909-921 (2012)

9. Brouwer, R.: Percolation, forest-fires and monomer dimers (or the hunt for self-organized criticality). PhD thesis, Vrije Universiteit (2005)

10. Damron, M., Sapozhnikov, A., Vágvölgyi, B.: Relations between invasion percolation and critical percolation in two dimensions. Ann. Probab. 37(6), 2297-2331 (2009)

11. Durrett, R.: Ten lectures on particle systems. In: Bernard, P. (ed.) Lectures on Probability Theory, vol. 1608. Lecture Notes in Mathematics, pp. 97-201. Springer, Berlin (1995)

12. Fortuin, C.M., Ginibre, J., Kasteleyn, P.W.: Correlation inequalities on some partially ordered sets. Commun. Math. Phys. 22, 89-103 (1971)

13. Garban, C., Pete, G., Schramm, O.: Pivotal, cluster and interface measures for critical planar percolation. J. Am. Math. Soc. 26, 939-1024 (2013)

14. Garban, C., Pete, G., Schramm, O.: The scaling limits of near-critical and dynamical percolation (2013). arXiv:1305.5526v2

15. Grimmett, G.: Percolation, 2nd edn. Springer, Berlin (1999) 
16. Kesten, H.: Scaling Relations for 2D-percolation. Commun. Math. Phys. 109, 109-156 (1987)

17. Kiss, D., Manolescu, I., Sidoraviciuos, V.: Ann. Probab, accepted for publication (2014)

18. Liggett, T.M.: Interacting Particle Systems. Springer, Berlin (2005)

19. Nolin, P.: Near-critical percolation in two dimensions. Electron. J. Probab. 13, 1562-1623 (2008)

20. Ráth, B.: Mean field frozen percolation. J. Stat. Phys. 137, 459-499 (2009)

21. Reimer, D.:Proof of the van den Berg-Kesten conjecture. Comb. Probab. Comput. 9(1), 27-32 (2000)

22. Russo, L.: On the critical percolation probabilities. Zeitschrift für Wahrscheinlichkeitstheorie und Verwandte Gebiete 56, 229-237 (1981)

23. Smirnov, S., Werner, W.: Critical exponents for two-dimensional percolation. Math. Res. Lett. 8, 729744 (2001)

24. Stockmayer, W.H.: Theory of molecular size distribution and gel formation in branched-chain polymers. J. Chem. Phys. 11, 45-55 (1943)

25. van den Berg, J., Brouwer, R.: Self-destructive percolation. Random Struct. Algorithms 24(4), 480-501 (2004)

26. van den Berg, J., de Lima, B.N.B., Nolin, P.: A percolation process on the square lattice where large finite clusters are frozen. Random Struct. Algorithms 40, 220-226 (2012)

27. van den Berg, J., Kesten, H.: Inequalities with applications to percolation and reliability. J. Appl. Probab. 22(3), 556-569 (1985)

28. van den Berg, J., Kiss, D., Nolin, P.: A percolation process on the binary tree where large finite clusters are frozen. Electron. Commun. Probab. 17(2), 1-11 (2012)

29. Wetherill, G.W.: Comparison of analytical and physical modeling of planetesimal accumulation. Icarus 88(2), 336-354 (1990) 